\title{
Visualization of the $\mathrm{Ca}^{2+}$-dependent regulation of voltage-gated Ether-à-go-go channels by FRET microscopy
}

\author{
PhD Thesis
}

in partial fulfillment of the requirements for the degree of Doctor of Philosophy $(\mathrm{PhD})$ in the Neurosciences graduate programme at the

Georg-August University Göttingen,

Faculty of Biology

Submitted by

José Tiago Gonçalves

from

Porto, Portugal 
First Referee: Prof. Dr. Walter Stühmer

Second Referee: Prof. Dr. Erwin Neher

Third Referee: Dr. Fred Wouters

Date of submission of the thesis: 29 May 2006

Date of public defense: 03 July 2006

Hereby I declare that I prepared this $\mathrm{PhD}$ Thesis "Visualization of the $\mathrm{Ca}^{2+}$ dependent regulation of voltage-gated Ether-à-go-go channels by FRET microscopy", on my own and with no sources and aids other than quoted.

Göttingen, 29 May 2006 


\section{Contents}

Abbreviations $\quad$ v

Introduction $\quad \mathbf{1}$

1.1 Ether-à-go-go voltage-gated potassium channels . . . . . . . . . . . 1

1.1.1 The eag phenotype and the Drosophila EAG channel . . . . . 1

1.1.2 Mammalian EAG channels . . . . . . . . . . . . . . . . 2

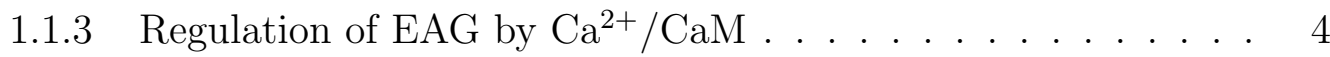

1.2 Fluorescence Resonance Energy Transfer (FRET) . . . . . . . . . . . 6

1.2.1 Use of genetically encoded Fluorescent Proteins in FRET assays 8

1.3 Aims of this work . . . . . . . . . . . . . . . 9

$\begin{array}{ll}\text { Materials and Methods } & 11\end{array}$

2.1 Materials ....................... . . 11

2.1.1 Solutions . . . . . . . . . . . . . . . . 11

2.1.2 Synthetic oligonucleotide primers . . . . . . . . . . . . . 12

2.1 .3 Plasmids . . . . . . . . . . . . . . . . . 13

2.1.4 Antibodies . . . . . . . . . . . . . . . . 14

2.2 Methods . . . . . . . . . . . . . . . . . . . . 14

2.2.1 Plasmid construction and mutagenesis . . . . . . . . . . . 14

2.2.2 Cell culture and transfection . . . . . . . . . . . . . . . 17

2.2 .3 Microscopy . . . . . . . . . . . . . . . . . . . 17

2.2.4 Image analysis and quantification . . . . . . . . . . . . . . . 18

2.2.5 Overlay assays . . . . . . . . . . . . . . . . . . 19

2.2.6 Generation of isoform specific antibodies against rEAG1 and rEAG2 ........................ . . 19

2.2.7 Primary culture of hippocampal neurons . . . . . . . . . . 20

2.2.8 Immunofluorescence . . . . . . . . . . . . . . . 21

Results $\quad \mathbf{2 3}$

3.1 Photoconversion product of YFP interferes with acceptor photobleaching measurement of FRET between Cyan and Yellow Fluorescent Proteins . . . . . . . . . . . . . . . . . . . . 23

3.2 FRET assay for the $\mathrm{Ca}^{2+}$-dependent interaction of EAG with CaM . 28 3.2.1 CaM binds directly to the N-terminus of EAG1 . . . . . . . . 32 
3.2.2 hEAG1 channels with mutated N-terminal (F151S, A152S) and C-terminal (F714S, F717S) CaMbd are unable to bind $\mathrm{CaM} \ldots \ldots \ldots \ldots \ldots \ldots$

3.2.3 The EAG2 homologue interacts with $\mathrm{CaM}$ in a $\mathrm{Ca}^{2+}$ dependent manner . . . . . . . . . . . . . . . 37

3.3 Study of the localization and $\mathrm{Ca}^{2+} / \mathrm{CaM}$ regulation of EAG in rat hippocampal cultures . . . . . . . . . . . . . . . . . . . 39

3.3.1 FRET measurements in neurons co-transfected with rEAG1Cerulean and Venus-CaM . . . . . . . . . . . . . 46

Discussion $\quad \mathbf{5 3}$

4.1 Photoconversion of Yellow Fluorescent Proteins . . . . . . . . . . . 53

4.2 Mechanism of $\mathrm{Ca}^{2+} / \mathrm{CaM}$ inhibition of EAG channels and potential functional implications . . . . . . . . . . . . . . 5 55

4.2.1 FRET assay for CaM binding to EAG . . . . . . . . . 56

4.2.2 N-terminal CaM binding domain of EAG1 channels . . . . . . 62

4.2.3 Molecular model of EAG1 inhibition by CaM . . . . . . . . . 64

4.2.4 EAG localization, inhibition and function . . . . . . . . . 66

$\begin{array}{ll}\text { Summary } & 69\end{array}$

$\begin{array}{ll}\text { Acknowledgments } & 79\end{array}$

$\begin{array}{lc}\text { Curriculum Vitæ } & 81\end{array}$ 


\section{Abbreviations}

apoCaM: apoCalmodulin

BFP: Blue Fluorescent Protein

CaMbd: Calmodulin binding domain

CaM: Calmodulin

CamK: Calmodulin-dependent protein Kinase

CFP: Cyan Fluorescent Protein

CNG: Cyclic Nucleotide Gated

cNMP: Cyclic Nucleotide Monophosphate

DIV: Days In Vitro

DMEM: Dulbeccos modified Eagle medium

E18: Embryonic day 18

EAG: Ether-à-go-go

ER: Endoplasmic Reticulum

FA: Formaldehyde

FCS: Fluorescence Correlation Spectroscopy

FGF : Fibroblast Growth Factors

FP: Fluorescent Protein

FRET: Fluorescence Resonance Energy Transfer
GABA: Gamma-Aminobutyric Acid

GAD: L-Glutamic acid decarboxylase

GFP: Green Fluorescent Protein

GST: Glutathione S-transferase

HRP: horseradish peroxidase

IgG: Immunoglobulin

IPTG: Isopropyl $\beta$-D-1-thiogalactopyranoside

MAP2: Microtubule Associated Protein2

NMDA: N-methyl-D-aspartate

PAC: PAS-associated C-terminal domain

PAS: Per-Arnt-Sim domain

PCR: Polymerase Chain Reaction

RT: Room Temperature

SEM: Standard Error of the Mean

SDS: Sodium Dodecyl Sulfate

SPR: Surface Plasmon Resonance

TCC: Tetramerised Coiled Coil

TEA: tetraethyl ammonium

XFP: photoconversion product of YFP/Venus

YFP: Yellow Fluorescent Protein 


\section{Introduction}

\subsection{Ether-à-go-go voltage-gated potassium chan- nels}

Ether-à-go-go (EAG or $\mathrm{KCNH}$ ) channels are non-inactivating voltage-gated $\mathrm{K}^{+}$ channels that are characterized by delayed-rectifier type currents with activation thresholds close to the membrane resting potential of most excitable cells $(\sim-40$ $\mathrm{mV}$ for hEAG1 and $-80 \mathrm{mV}$ for hEAG2). They are thought to play a role in the regulation of neuronal excitability [8,21,31] and in oncogenesis [59].

\subsubsection{The eag phenotype and the Drosophila EAG chan- nel}

The EAG gene owes its name to the phenotype of mutant Drosophila melanogaster which displayed rapid shaking of legs following etherization [31]. This behaviour was immediately associated with a change in neuronal excitabilty causing "overstimulation of the muscles controlling shaking or the removal of a normally present inhibitory influence" [31]. Work by Ganetzky and Wu [21] linked the eag mutation to TEA-sensitive potassium currents in the Drosophila neuromuscular junction and reinstated the hypothesis that these currents play a role in maintaining normal neuronal excitability. The eag locus was found to encode a polypeptide with similarities to voltage-gated ion channels [88] and Brüggemann et al. [8] showed that EAG encodes a novel voltage-gated ion channel that is permeable for potassium and calcium and modulated by cAMP. 


\subsubsection{Mammalian EAG channels}

Ludwig et al. cloned the rat homologue of eag (rEAG1) based on sequence similarities with the Drosophila channel [38], 61\% of the aminoacid sequence of the channel was identical across both species, in particular in the regions of the N-terminus and trans-membrane domains (Fig.1.1). However, several properties of rEAG1 currents differed from those of the Drosophila channel [38]. Mammalian EAG1 channels do not mediate inward $\mathrm{Ca}^{2+}$ currents and their activation kinetics had an increased dependency on the membrane holding potential. Moreover, mamalian channels were not regulated by cyclic nucleotides, whereas in Drosophila application of cAMP caused a significant increase in outward current amplitudes [8]. The subsequently cloned mouse and human EAG1 [55,62], rEAG2 [39] and hEAG2 [28] share these electrophysiological charcteristics to a large extent, although the EAG2 isoforms have much lower activation thresholds $(\sim-80 \mathrm{mV})$ and slower activation. A defining characteristic of EAG currents is the strong dependence of their activation kinetcs on the holding potential (Cole-Moore effect [15]). Hyperpolarization slows the the activation of the channel, whereas depolarization accelerates it. This effect is enhanced by the presence of $\mathrm{Mg}^{2+}$ in the extracellular medium [78].

EAG channels are the founding members of the the KCNH or Ether-à-go-go related family of voltage-gated $\mathrm{K}^{+}$channels [89] which currently comprises eight members (Table 1.1), classified under three subfamilies: EAG, ERG (EAG Related Gene) and ELK (EAG Like). EAG-family channels are structurally characterized by the presence of the highly conserved EAG or PAS (Per-Arnt-Sim) domain in their N-termini [10]. Their currents, however, can differ significantly from those of EAG.

Table 1.1 The KCNH (eag-related) family of human genes

\begin{tabular}{|c|c|c|c|}
\hline Gene name & IUPHAR channel name & Other gene names & Chrom. location \\
\hline KCNH1 & Kv10.1 & heag1, ether-à-go-go & $1 q 32.1-32.3$ \\
\hline $\mathrm{KCNH} 2$ & Kv11.1 & herg1, ether-à-go-go related & $7 q 35-36$ \\
\hline $\mathrm{KCNH} 3$ & Kv12.2 & helk2, ether-à-go-go like & $12 q 13$ \\
\hline $\mathrm{KCNH} 4$ & Kv12.3 & helk3, ether-à-go-go like & $17 q 21.31$ \\
\hline KCNH5 & Kv10.2 & heag2, ether-à-go-go & $14 \mathrm{q} 24.3$ \\
\hline KCNH6 & Kv11.2 & herg2, ether-à-go-go related & 17 \\
\hline $\mathrm{KCNH} 7$ & Kv11.3 & herg 3 , ether-à-go-go related & $2 q 24.3$ \\
\hline $\mathrm{KCNH} 8$ & Kv12.1 & helk1, ether-à-go-go like & $3 \mathrm{p} 24.3$ \\
\hline
\end{tabular}




\section{Expression}

Rat EAG channels are expressed exclusively in the brain [38, 39, 59]. In-situ hybridization studies $[39,66]$ have detected rEAG1 transcripts predominantly in the hippocampus, cerebral cortex, olfactory bulb and granular layer of the cerebellum of adult rats. rEAG2 is present the hippocampus, cerebral cortex and thalamus, but only to a small extent in the cerebellum. In rat hippocampal cultures EAG1 was found to be present in glutamatergic post-synapses, whereas EAG2 was localized in GABAergic post-synapses [25].

\section{Structural characteristics}

Functional EAG channels are tetramers but, unlike most voltage-gated $\mathrm{K}^{+}$channels, the domain mediating the tetramerization of EAG (Tetramerizing Coiled Coil or TCC domain) is located at the C-terminus of the channel subunits [26,37]. hEAG1 and hEAG2 have been shown to form functional heterotetramers upon co-expression, resulting in heteromeric channels where the slow activation kinetics of hEAG2 are dominant [69].

EAG channel subunits are multipass membrane proteins with six trans-membrane segments (S1-S6) and intracellular tails at the N- and C-termini, a standard topology which is shared by other voltage-gated $\mathrm{K}^{+}$channels $[88,89]$. The voltage sensor is located in the positively-charged S4 domain, whereas the channel pore is lined by transmembrane segments S5 and S6.

The cytosolic N-terminus of EAG contains a Per-Arnt-Sim (PAS) domain followed by a PAC sequence (PAS-associated C-terminal), which is commonly present at the C-terminus of PAS domains [98]. PAS domains are conserved structural motifs which are thought to have a chemosensing and signal transduction function [86,98]. The function of the PAS domain in EAG-family channels is not entirely clear, although it has been suggested that it may play a role in channel gating by binding to the S4-S5 intracellular loop $[10,77]$. The extracellular S5-S6 loop contains two asparaginelinked glycosylation sites (N388, N406), which have been shown to be necessary for correct membrane expression and channel function [52].

The cytoplasmic region downstream of S6 comprises approximately $50 \%$ of the aminoacid sequence of EAG channels and contains three known regulatory domains - a cyclic nucleotide (cNMP) binding domain [89], a CaM binding domain 


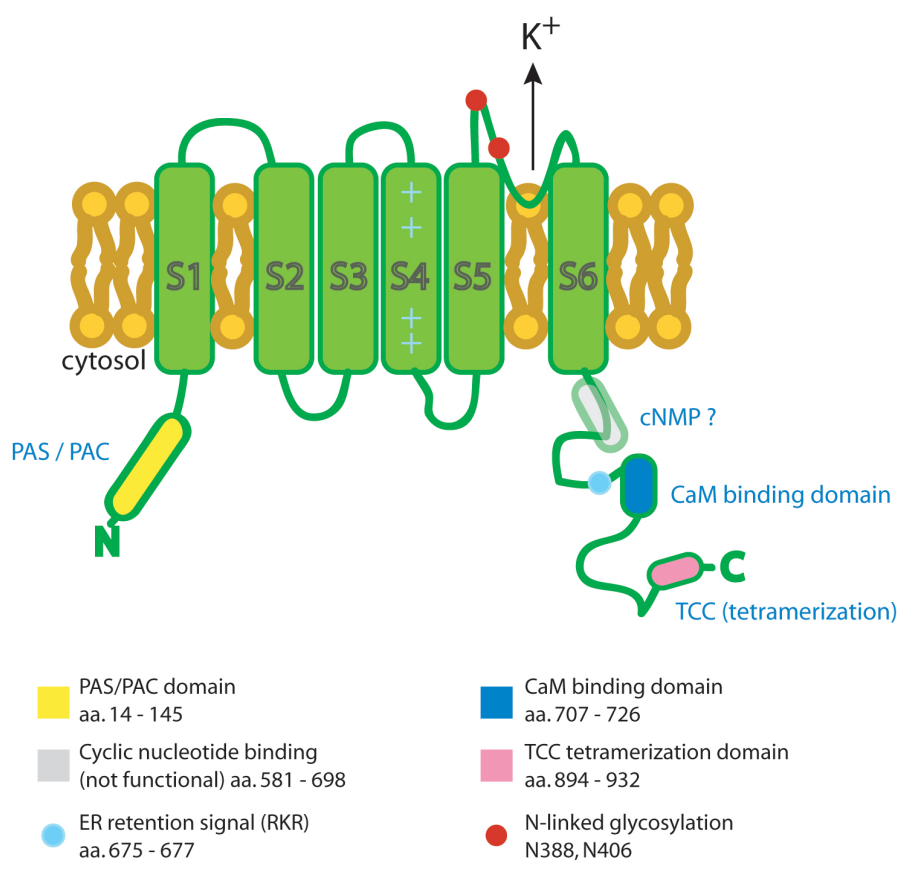

Figure 1.1: Scheme of hEAG1 membrane topology, including known functional domains and structural features.

(CaMbd) [70] and a Tetramerizing Coiled Coil (TCC). A three aminoacid endoplasmatic reticulum (ER) retention signal $\left({ }_{675} \mathrm{RKR}_{677}\right)$ is thought to prevent channel trafficking prior to correct assembly $[26,33]$.

Although it contains a segment with substantial similarities to the cNMP binding domain of cyclic nucleotide gated $(\mathrm{CNG})$ channels, mammalian EAG is not modulated by cyclic nucleotides [38], unlike its Drosophila homologue [8]. This cNMP binding motif appears therefore to be non-functional.

\subsubsection{Regulation of EAG by $\mathrm{Ca}^{2+} / \mathrm{CaM}$}

rEAG1 was found to be inhibited by intracellular $\mathrm{Ca}^{2+}$ with a half-maximal inhibition of $\sim 100 \mathrm{nM}$ [74]. This inhibition is mediated by the direct binding of Calmodulin (CaM), a $\mathrm{Ca}^{2+}$-binding protein, to the C-terminus (aa. 707-726) of the channel [70]. CaM binds hEAG1 only in its $\mathrm{Ca}^{2+}$-bound form, in contrast with other potassium channels where CaM is a constitutive channel subunit [67]. The binding of one CaM molecule was found to be sufficient for $\mathrm{Ca}^{2+}$-induced channel closure to occur [70].

Interestingly, Drosophila EAG currents are enhanced by $\mathrm{Ca}^{2+} / \mathrm{CaM}$ KinaseII 
(CaMKII) phosphorylation [87], which implies that $\mathrm{Ca}^{2+}$ is exerting opposing effects in mammalian and Drosophila EAG.

The $\mathrm{Ca}^{2+}$ regulation of ion channels provides a link between intracellular signaling pathways and membrane electrical activity that can play a role in regulating $\mathrm{Ca}^{2+}$ levels, neuronal excitability and synaptic release. Other $\mathrm{K}^{+}$channels are known to be modulated by $\mathrm{Ca}^{2+} / \mathrm{CaM}$, namely $\mathrm{Ca}^{2+}$-activated $\mathrm{K}^{+}$channels (IK and SK), CNG and KCNQ channels [67].

\section{CaM binding domains and regulation of ion channels}

Calmodulin is a small (148 aa., $17 \mathrm{kDa}$ ), soluble $\mathrm{Ca}^{2+}$-binding protein that is highly conserved in eukaryotes. It is extremely abundant, with predominance in the brain where it can account for $0.5 \%$ of all proteins and reach concentrations of $1-10 \mu \mathrm{M}$ $[67,80]$. Intracellularly, CaM is present throughout the cytosol and nucleus [16] but concentrates mostly in the regions adjacent to the plasma membrane [67].

Structurally, CaM is a monomer with two pairs of $\mathrm{Ca}^{2+}$-binding EF-hand domains forming two lobes at opposing ends of the protein [2]. Isolated CaM binds $\mathrm{Ca}^{2+}$ with a dissociation constant $\left(\mathrm{K}_{d}\right)$ of approximately $5 \cdot 10^{-6} \mathrm{M}$ but its affinity can be much higher when in complex with an enzyme [29,67]. Upon binding of $\mathrm{Ca}^{2+}$ a hydrophobic interaction sites are exposed in each lobe. $\mathrm{Ca}^{2+}$-dependent binding occurs through hydrophobic interactions and salt bridges, the two lobes of CaM encircling the target binding site [43]. CaM binding domains are therefore frequently amphipathic alpha-helices with a positively charged hydrophilic side [67].

Although there is no consensus sequence for CaM binding domains, most target peptides can be classified as containing one of three motifs [60]:

- IQ motifs - bind CaM independently of $\mathrm{Ca}^{2+}$. Present in some ion channels which constitutively bind CaM, such as the L-type $\mathrm{Ca}^{2+}$ channel. CaM binding domains with similarities to the IQ motif are present in IK and SK channels, which also bind CaM constitutively [67]. The CaM binding domain in the C-terminus of EAG has significant similarities to the latter, although it binds CaM only in its $\mathrm{Ca}^{2+}$-bound form [70].

- 1-8-14 motifs - bind CaM in its $\mathrm{Ca}^{2+}$-bound state. Their name refers to the position of conserved hydrophobic aminoacids in an amphipathic helix structure. 1-8-14 motifs tend to have a high affinity for $\mathrm{Ca}^{2+} / \mathrm{CaM}$. Present e.g. in 
CNG channels [67]

- 1-5-10 motifs - similar to 1-8-14, bind CaM in its $\mathrm{Ca}^{2+}$-bound state. These motifs are present e.g. in NMDA receptors

\subsection{Fluorescence Resonance Energy Transfer (FRET)}

FRET is the term used to describe the non-radiative transfer of energy between two fluorophores (termed 'acceptor' and 'donor' molecule) in close vicinity [20]. In its applications for biological assays, two molecules of interest are labelled with fluorescent molecules with overlaying emission and absorption spectra. FRET can be detected only when the labelled molecules come within a very short distance of each other - typically $\sim 5 \mathrm{~nm}$, thus implying that the two molecules are interacting.

\section{Fluorescence}

Fluroescence is a property of chemical species (fluorophores) that have photoexcitable electrons and are able to re-emit a light photon in a time scale of the order of nanoseconds.

Photoexcitable electrons in fluorescent molecules (usually singlet $\pi$ electrons) are excited into a higher energy level by the electric field component of incoming electromagnetic radiation.

When the electron decays back to the ground state a photon is emited. This photon has lower energy than the excitation photon - a part of the absorbed energy is converted internally in the molecule as the excitable electron drops to degenerate vibrational and rotational energy states. This difference between absorption and emission spectra is called Stokes' shift.

Decay into the ground state is not the only possibility for excited electrons. The increase in bond energy can trigger a chemical reaction, such as oxydation, which would destroy the fluorophore - this process is called photobleaching. A second possibility is the transfer of energy to another fluorophore by FRET.

The average time span between absorption and emission is called the fluorescence lifetime of the fluorophore and can be calculated from the rates of radiative decay 
$\Gamma$ and of non-radiative decay $K_{n r}$ (e.g. FRET and photobleaching) [20,35]:

$$
\tau=\frac{1}{\Gamma+K_{n r}}
$$

\section{Mathematical formalism for FRET}

The energy transfer efficiency $(E)$ between an excited donor and an acceptor molecule is strongly dependent on distance, as can be inferred from the mathematical description of the process $[20,35]$ :

$$
E=\frac{R_{0}^{6}}{R_{0}^{6}+r^{6}}
$$

Where $r$ is the separation between donor and acceptor and $R_{0}$ is the distance at which the energy transfer efficiency is $50 \%$, i.e. the rate of transfer is equal to the decay rate of the donor $[20,35]$ :

$$
R_{0}^{6}=\frac{9000(\ln 10) \kappa^{2} Q_{d}}{128 \pi^{4} N n^{4}} \int_{0}^{\infty} \frac{F_{d}(\bar{\nu}) \epsilon_{a}(\bar{\nu}) d \bar{\nu}}{\bar{\nu}^{4}} d \bar{\nu}
$$

Where:

- $\kappa$ is a term describing the relative orientation of the fluorophore dipoles. When the orientation is the same, energy transfer is optimal. Perperdicular orientations do not result in energy transfer.

- $Q_{D}$ is the 'quantum efficiency' of the donor molecule $\left(Q_{d}=\Gamma /\left(\Gamma+K_{n r}\right)\right.$

- $n$ is the refractive index of the medium.

- $N$ is Avogadro's number

- The integral to the right describes the spectrum overlap between acceptor and donor fluorophores. The energy accepted must be in the same range as the energy donated - must therefore choose fluorophores with emission (donor) and excitation (acceptor) spectral overlap.

The requirement for spectral overlap leads to difficulties in the detection of FRET. Overlapping spectra usually mean that the acceptor molecule will also be mildly excited by the donor excitation light and that the emission of the donor will extend into the emission spectra of the acceptor. Detecting the sensitized (non-photoexcited) 
emission of the acceptor is therefore not an ideal method for detecting FRET, unless spectral fingerprinting techniques are used for determining the individual emissions of donor and acceptor fluorophores [53,54,100].

Other effects of FRET can be used for estimating FRET efficiencies, namely

- The decrease in intensity of the donor

- Decrease of donor lifetime

- Decrease in anisotropy (polarization) of emitted donor light

- Delayed photobleaching dynamics

\section{Measurement of FRET by acceptor photobleaching}

Acceptor photobleaching [4] is a technically simple and widely used method to estimate energy transfer efficiencies $(E)$ based on the decrease in donor intensity due to FRET. In acceptor photobleaching measurements the intensity of donor fluorescence is recorded in a sample with both donor and acceptor molecules, and the same measurement is repeated after the destruction of the acceptor by photobleaching. The difference between donor fluorescence before and after photobleaching is then normalized to the intensity of the donor after photobleaching in order to calculate the FRET efficiency [4]:

$$
E=\frac{I_{\text {DONORpost-bleach }}-I_{\text {DONORpre-bleach }}}{I_{\text {DONORpost-bleach }}}
$$

\subsubsection{Use of genetically encoded Fluorescent Proteins in FRET assays}

The availability of different genetically encoded Fluorescent Proteins (FPs) has radically broadened the applications of FRET over last decade (see [9] for a review). The first reports of FRET between FPs used the Blue Fluorescent Protein (BFP) as donor and GFP as acceptor [45]. However, BFP suffered from low molar extinction coefficient, low quantum yield and high susceptibility for photobleaching. CFP proved to be superior in the latter two aspects and was therefore quickly adopted as a FRET donor with YFP as acceptor [46]. This is currently the most widely used 
genetically encoded FRET pair, although usually in the form of the brighter and faster maturing variants Cerulean [61] and Venus [51].

Nevertheless, CFP-YFP is not an optimal FRET pair. There is significant overlap of the emission spectra of both fluorophores [9] and this problem is exacerbated by the low absorbance and quantum yield of $\mathrm{CFP} /$ Cerulean which results in YFP/Venus being up to 5 times brighter than the cyan donor [61]. Furthermore, the $430 \mathrm{~nm}$ lasers needed for optimal excitation of CFP are not yet widely available, which aggravates the brightness problem. Additionally, CFP has its emission maximum in a spectral region with high autofluorescence from cellular components [6]. Several attempts have been made at developing new genetically encoded FRET pairs, however, most of the FPs proposed so far have disadvantages in comparison to CFP-YFP. Most notably, red fluorescent proteins, which could serve as acceptors for GFP, have been plagued with problems such as slow chromophore maturation [84], tetramerization [13], and low photostability [71]. Therefore, CFP/Cerulean - YFP/Venus remains the FRET pair of choice for most biological applications.

\subsection{Aims of this work}

EAG1 channels were found to be inhibited by $\mathrm{Ca}^{2+} / \mathrm{CaM}$ at concentrations close to the resting $\mathrm{Ca}^{2+}$ levels of neurons [70], a feature which can have far-reaching implications for their proposed function as regulators of neuronal excitability.

The present work aims at:

Gaining a better understanding of the molecular mechanism of EAG inhibition by $\mathrm{Ca}^{2+} / \mathrm{CaM}$. A FRET assay for the binding of CaM to the channel is to be established.

Investigating the sub-cellular localization of EAG in neurons. And its relation with the functional role of the channel, in particular in what regards the $\mathrm{Ca}^{2+} /$ CaM-mediated inhibition. 


\section{Materials and Methods}

\subsection{Materials}

\subsubsection{Solutions}

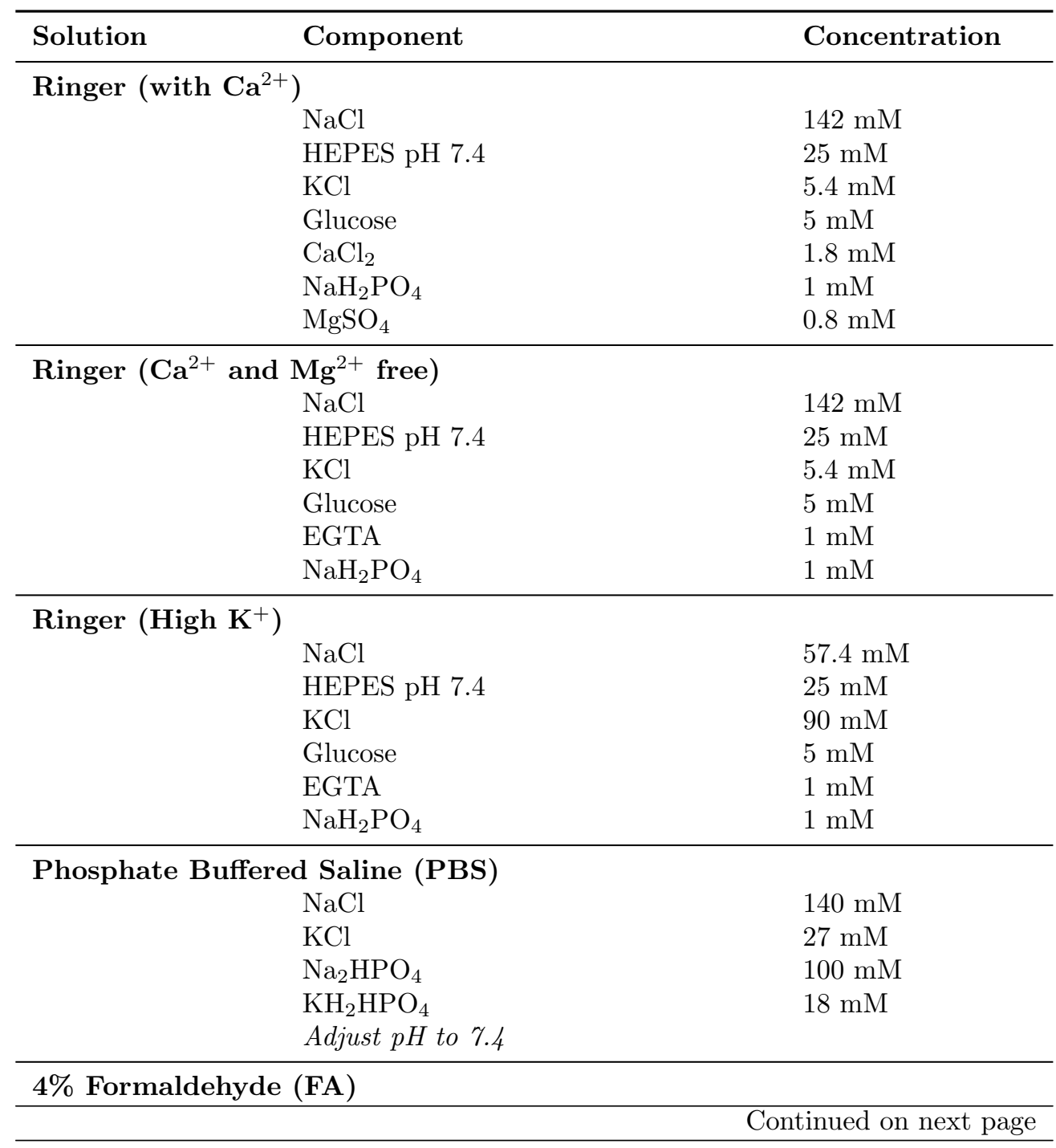


Table 2.1 - continued from previous page

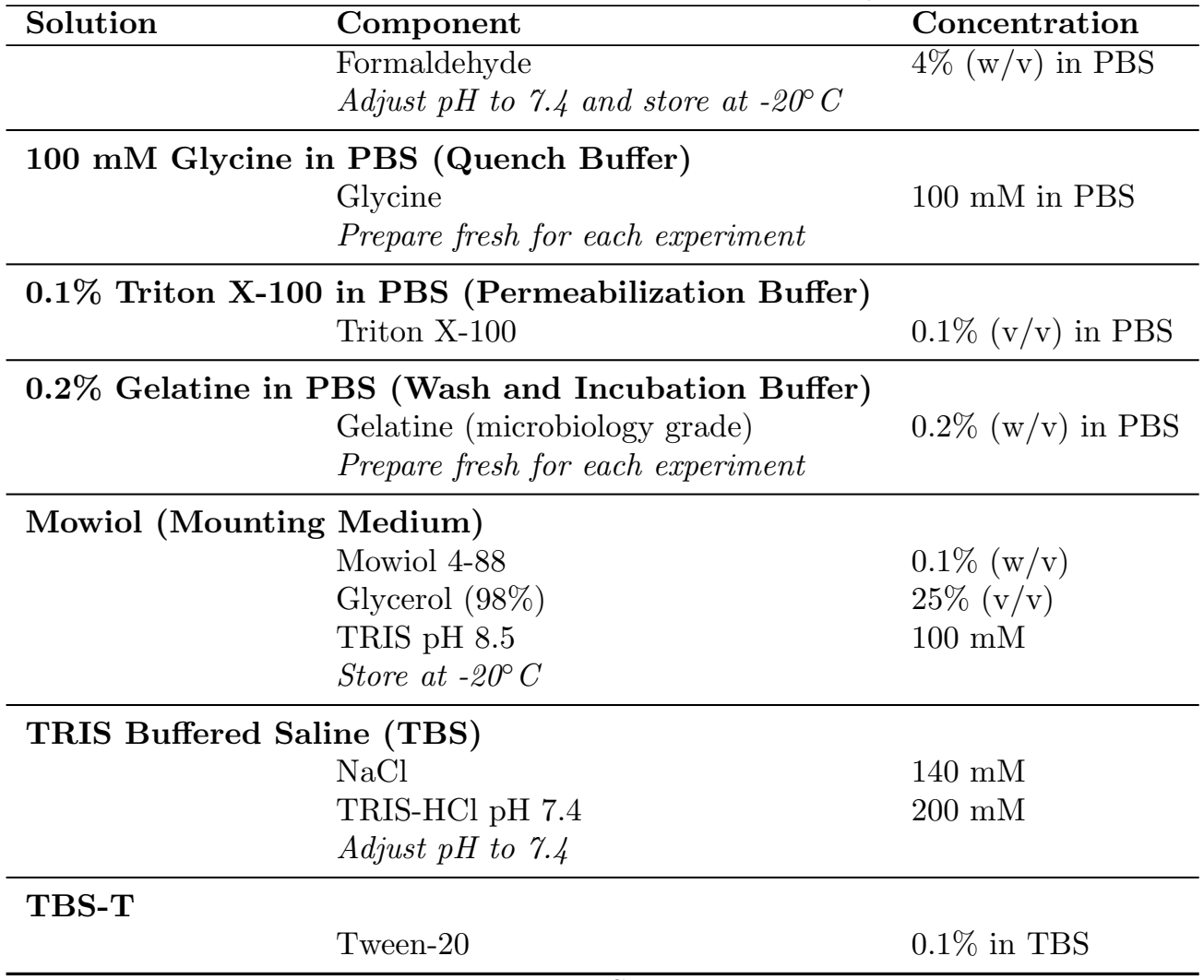

Table 2.1: Solutions

\subsubsection{Synthetic oligonucleotide primers}

All DNA primers used in this work are listed in Table 2.2. For primers used in mutagenesis PCR reactions the mutant base pairs are denoted by the use of uppercase letters.

\begin{tabular}{ll}
\hline Primer name & Sequence $\left(5^{\prime} \rightarrow 3^{\prime}\right)$ \\
\hline $2182(\mathrm{fw})$ & cgcggatccgcggccgccatggtgagcaagggcgaggag \\
$2183(\mathrm{rv})$ & atagtttagcggccgctcacttgtacagctcgtccatgccgagag \\
$2325(\mathrm{fw})$ & tacgtaatgcggccgccatggtgagcaagggcgagga \\
$2326(\mathrm{rv})$ & cgggatccttacttgtacagctcgtccatgc \\
$2363(\mathrm{fw})$ & ataagaatgcggccgcatggctgaccaactgactgaag \\
BamHINterm-fw & cgcggatccatgaccatggctggc \\
camnot-rv & ataagaatgcggccgcctacttcgctgtcatcatttgtac \\
fw_cam_mut_h & ccggcgcctcTCCcagagaTCCcgacagcagaaagagg \\
rv_cam_mut_h & cctctttctgctgtcgGGAtctctgGGAgaggcgccgg \\
fw_cam_mut_r & caggaggctcTCCcaaaggTCCcgccagcagaaagaag \\
\hline &
\end{tabular}


Table 2.2 - continued from previous page

\begin{tabular}{ll}
\hline Primer name & Sequence $\left(5^{\prime} \rightarrow 3^{\prime}\right)$ \\
\hline rv_cam_mut_r & cttctttctgctggcgGGAcctttgGGAgagcctcctg \\
Nterm-fw_40 & cgcggatccatgcagaacacatttctggagaaca \\
Nterm-fw_208 & cgcggatccatgtatggagagctgaccg \\
Nterm-fw_436 & cgcggatccatgaaaggttgggggaagtttgc \\
Nterm-rv_288 & atagtttagcggccgcttcgaaggagttcatctcg \\
Nterm-rv_441 & atagtttagcggccgcacctttgcaggagtcgtc \\
Nterm-rv_627 & atagtttagcggccgcgatgtgagggggtgtctttgg \\
fw_NtermMut1 & gcagcaggggaTCcTCgcagcagctggccccc \\
rv_NtermMut1 & gggggccagctgctgcGAgGAtcccctgctgc \\
fw_NtermMut2 & ggttgggggaagtCtTctcgactgacgagagc \\
rv_NtermMut2 & gctctcgtcagtcgagAaGacttcccccaacc \\
fw_NtermMut3 & cagaagggtgagaatTCtGacaagcactcgcgcctgg \\
rv_NtermMut3 & ccaggcgcgagtgcttgtCaGAattctcacccttctg \\
fw_NtermMut2_h & ggctgggggaagtCtTctcggctgacaagagc \\
rv_NtermMut2_h & gctcttgtcagccgagAaGacttcccccagcc \\
NtermNotI-rv & atagtttagcggccgctgtggtcttaaagacacag \\
vencam-fw & ctactagctagcatggtgagcaagggcgag \\
\hline
\end{tabular}

Table 2.2: Oligonucleotide primers

\subsubsection{Plasmids}

- pcDNA3 Invitrogen

- pGEX-4T-1 Amersham Pharmacia Biotech

- C-Cerulean-pcDNA3 Same as pcDNA3 but with the DNA encoding for the Cerulean fluorescent protein [61] cloned between NotI restriction sites at the $3^{\prime}$ end of the multiple cloning site.

- pCB6-C-GFP Mammalian expression vector (CMV promoter). This plasmid is a gift of Dr. M. Way (CRUK, London, UK) [49]. It is used to make C-terminus GFP-fusion constructs. GFP is flanked by NotI and BamHI restriction sites.

- pCB6-N-YFP Similar to pCB6-C-GFP but designed to make N-terminus YFP-fusion constructs of the gene of interest. YFP is flanked by KpnI and NotI restriction sites.

- pMH4-I-SYN Mammalian expression vector (human Synapsin promoter). This plasmid is a gift of Dr. S. Kügler (U.K.G., Göttingen). The Synapsin promoter enables selective expression in neuronal cells [32]. 


\subsubsection{Antibodies}

\begin{tabular}{|c|c|c|c|c|}
\hline Antigen & Host & Type & Provider & Concentration \\
\hline \multicolumn{5}{|l|}{ Neuronal Markers } \\
\hline MAP2 & Mouse & Monoclonal & Sigma (M4403) & $1: 500$ \\
\hline p38 (Synaptophysin) & Mouse & Monoclonal & Sigma (S5768) & $1: 2000$ \\
\hline \multicolumn{5}{|l|}{ Calmodulin } \\
\hline \multicolumn{5}{|l|}{ EAG } \\
\hline rEAG1 (7194) & Rabbit & Polyclonal & see $\S 2.2 .6$ & $\sim 3 \mu \mathrm{g} / \mathrm{ml}$ \\
\hline rEAG2 (7205) & Rabbit & Polyclonal & see $\S 2.2 .6$ & $\sim 3 \mu \mathrm{g} / \mathrm{ml}$ \\
\hline \multicolumn{5}{|c|}{ Fluorophore-coupled secondary antibodies } \\
\hline Rabbit IgG (H+L) & Goat & FITC-conj. & Jackson (111095003) & $1: 5500$ \\
\hline Rabbit IgG $(\mathrm{H}+\mathrm{L})$ & Goat & Cy3-conj. & Jackson (111165003) & 1:5000 \\
\hline Mouse IgG $(\mathrm{H}+\mathrm{L})$ & Goat & Cy5-conj. & Jackson (115175003) & $1: 3500$ \\
\hline
\end{tabular}

\subsection{Methods}

\subsubsection{Plasmid construction and mutagenesis}

The following subsections describe the origin or cloning procedure of the cDNA constructs used in this work. Whenever the Polymerase Chain Reaction (PCR) was used in the cloning process, the fidelity of the resulting constructs was verified by DNA sequencing (MPI for Experimental Medicine, DNA core-facility).

\section{hEAG1-Cerulean}

The C-terminal Cerulean-fusion construct of full-length hEAG1 was cloned from CB6-hEAG1-GFP (C-terminal GFP-fusion construct. E. Herrero, G. Bunt). Cerulean [61] was amplified by PCR with flanking NotI and BamHI sites (primers 2325,2326 ) and subsequently cloned in place of GFP into the CB6-hEAG1 vector $[7,49]$. 


\section{rEAG1-GFP/rEAG1-Cerulean and rEAG2-GFP/rEAG2-Cerulean}

The C-terminal GFP-fusion constructs of full-length rEAG1 and rEAG2 (pcDNA3rEAG1-GFP, pcDNA3-rEAG2-GFP) were obtained from M. Ninkovic and G. Bunt. In order to generate the cyan fluorescent fusion constructs, Cerulean [61] was amplified by PCR with flanking NotI sites at both ends (primers 2182, 2183) and cloned in place of GFP into the pcDNA3-rEAG1 and pcDNA3-rEAG2 vectors.

The neuronal-expression, GFP-fusion construct of rEAG1 (pMH4-I-SYN-rEAG1GFP) was cloned by J. Uhlendorf and G.Bunt. In order to generate a cyan fluorescent fusion construct of rEAG1 under the control of the synapsin promoter (hSyn rEAG1-Cerulean), Cerulean [61] was amplified by PCR with flanking NotI sites at both ends (primers 2182, 2183) and cloned in place of GFP into the pMH4-I-SYNrEAG1 vector.

\section{Cterm-Cerulean}

The Cerulean- and Venus- fusion constructs of the C-terminus (M478-S962) of rEAG1 was cloned from pcDNA3-Cterm-rEAG1-GFP (C-terminal GFP-fusion construct. M. Ninkovic, G.Bunt). Cerulean [61] and Venus were amplified by PCR with flanking NotI sites at both ends (primers 2182, 2183) and cloned in place of GFP into the pcDNA3-Cterm vector.

\section{GST-fusion constructs of rEAG1}

Fragments of the cytosolic N-terminus of rEAG1 were obtained by PCR amplification of the cDNA coding for the following segments: M1-T219 (N-term); M1-I209 (A); M1-G147 (B); M1-E96 (C); Q14-T219 (D); M70-T219 (E), K146-T219 (F). The C-terminus (M478-S962) fragment was obtained by digestion of C-term-Cerulean with BamHI and NotI restriction enzymes. The fragments were then subcloned between BamHI and NotI restriction sites in the pGEX-4T-1 vector (GST-fusion, Pharmacia). The primers used for the amplification of the rEAG1 fragments are presented in Table 2.4 . 


\begin{tabular}{lcc}
\hline \hline & Forward primer & Reverse primer \\
\hline N-term-rEAG1 & BamHINterm-fw & NtermNotI-rv \\
Fragment A & BamHINterm-fw & Nterm-rv_627 \\
Fragment B & BamHINterm-fw & Nterm-rv_441 \\
Fragment C & BamHINterm-fw & Nterm-rv_288 \\
Fragment D & Nterm-fw_40 & NtermNotI-rv \\
Fragment E & Nterm-fw_208 & NtermNotI-rv \\
Fragment F & Nterm-fw_436 & NtermNotI-rv \\
\hline
\end{tabular}

Table 2.4: Primers used for the amplification of rEAG1 fragments

\section{YFP-CaM and YFP-apoCaM}

The cDNA for rat Calmodulin II (CaM), a kind gift of Dr. M. Shea (Univ. of Iowa, USA), was amplified by PCR with flanking NotI restriction sites (primers 2363, camnot_rv) and subsequently cloned into the CB6-N-YFP vector to generate N-terminus YFP-labeled Calmodulin (YFP-CaM). N-terminus YFP-labeled apoCalmodulin (YFP-apoCaM [50]) was a gift of Dr. D. Yue (Johns Hopkins Univ., USA). Mutations in all EF-hand domains render this version of CaM insensitive to $\mathrm{Ca}^{2+}$.

\section{(hSyn) Venus-CaM and Venus-apoCaM}

In order to generate Venus-fusion [51] constructs of CaM and apoCaM for neuronal expression, Venus was amplified by PCR with flanking NheI and NotI sites (primers vencam-fw, 2183) and cloned into the pMH4-I-SYN vector. The cDNA cloning for CaM and apoCaM, obtained by digesting the pcDNA3-based CaM constructs with the NotI restriction enzyme, was subsequently cloned at the $3^{\prime}$ end of Venus to obtain the pMH4-I-SYN-Venus-CaM and apoCaM vectors.

\section{Introduction of point-mutations in the CaM-binding domains of EAG1}

Point mutations of the Calmodulin binding-sites of rat and human EAG1 were generated using the PCR-based QuikChange mutagenesis kit (Stratagene), following the instructions of the manufacturer.

Mutations F714S,F717S (described in [70]) were introduced into rEAG1 constructs using primers fw_cam_mut_r, rv_cam_mut_r, and in their human counterparts using primers fw_cam_mut_h, rv_cam_mut_h. 
The pairs of mutations F151S,A152S; V164S, L165S and V178S,H179D were introduced in the N-terminus CaM-binding domains of hEAG1 and rEAG1 constructs using the primers shown in Table 2.5.

\begin{tabular}{llcc}
\hline \hline Mutations & Target & Forward primer & Reverse primer \\
\hline F151S, A152S & rEAG1 & fw_NtermMut2 & rv_NtermMut2 \\
F151S, A152S & hEAG1 & fw_NtermMut2_h & rv_NtermMut2_h \\
V164S, L165S & rEAG1 & fw_NtermMut1 & rv_NtermMut1 \\
V178S, H179D & rEAG1 & fw_NtermMut3 & rv_NtermMut3 \\
\hline
\end{tabular}

Table 2.5: Primers used in mutagenesis of N-terminus CaM-binding domains

\subsubsection{Cell culture and transfection}

HEK293 cells were cultured in DMEM:F12 (1:1) medium (Gibco), supplemented with 10\% FCS and $0.1 \%$ streptomycin/penicillin (Gibco) under $5 \% \mathrm{CO}_{2}$ and humidified conditions. Cells were plated on Poly-L-Lysine-coated glass coverslips and transfected at 20-30\% confluency using FuGene6 (Roche), according to the instructions of the manufactrer. The total amount of cDNA used was $0.5 \mu \mathrm{g}$ per 15 $\mathrm{mm}$ cover slip. For co-transfections a cDNA ratio of $3: 1(\mathrm{~m} / \mathrm{m})$ was used $(\mathrm{EAG}$ (donor):CaM (acceptor)).

\section{$\mathrm{Ca}^{2+}$-ionophore treatment}

For the CaM-binding FRET assay, cells were treated 36 hrs after transfection with $1 \mu \mathrm{M}$ ionomycin (Calbiochem) in either Ringer solution with $\mathrm{Ca}^{2+}\left("+\mathrm{Ca}^{2+}\right.$ ") or $\mathrm{Ca}^{2+}$-free Ringer with $1 \mathrm{mM}$ EGTA ("-Ca ${ }^{2+}$ "), for 10 min at $37^{\circ} \mathrm{C}$ and subsequently fixed for $25 \mathrm{~min}$ with $4 \%$ formaldehyde. After fixation cells were briefly washed in PBS and mounted in Mowiol.

\subsubsection{Microscopy}

Cells were imaged on a Leica DMIRE2 inverted microscope equipped with a TCS SP2 (AOBS) confocal scanner and a 63x NA1.4 HCX PL Apo objective (Leica). The excitation laser lines and spectral collection window for each fluorophore are detailed in Table 2.6. Spectral scans were acquired using $405 \mathrm{~nm}$ excitation-light and 
Table 2.6: List of fluorophores and respective spectral settings

\begin{tabular}{lcccc}
\hline \hline Fluorophore & $\begin{array}{c}\text { Excitation max. } \\
(\mathrm{nm})\end{array}$ & $\begin{array}{c}\text { Emission max. } \\
(\mathrm{nm})\end{array}$ & $\begin{array}{c}\text { Laser line } \\
(\mathrm{nm})\end{array}$ & $\begin{array}{c}\text { Collection range } \\
(\mathrm{nm})\end{array}$ \\
\hline Cerulean [61] & 433 & 470 & $405 / 458$ & $470-505$ \\
GFP [82] & 488 & 505 & 488 & $500-535$ \\
YFP / Venus [51] & 515 & 528 & 514 & $525-610$ \\
Cy3 & 550 & 570 & 561 & $575-625$ \\
Cy5 & 649 & 670 & 633 & $652-730$ \\
\hline
\end{tabular}

a $4.5 \mathrm{~nm}$ spectral acquisition window, which corresponds to the minimal spectral resolution of the Leica TCS SP2 (AOBS) scanner. The step-size for spectral scans was equally set to $4.5 \mathrm{~nm}$.

\section{FRET measurements}

Cerulean and YFP / Venus were excited using, respectively, the 458 and $514 \mathrm{~nm}$ lines of an Argon laser. Cerulean emission was recorded in the 470-505 nm range and YFP between 525-610 nm. This choice of Cerulean emission range produced images with practically complete spectral separation from YFP emission. Photomultiplier gain and offset values, as well as laser intensities were kept constant for all images acquired of a given cell, before as well after bleaching of the acceptor fluorophore. The pinhole opening was maximally opened during acquisition (5.2 Airy units). Fluorescence Resonance Energy Transfer (FRET) was measured by the method of Acceptor Photobleaching [4]. Images of both donor and acceptor constructs were acquired before and after photo-destruction of YFP to $15 \%$ of its initial intensity, in a region of interest inside a cell.

\subsubsection{Image analysis and quantification}

Image analysis was performed with Matlab (Mathworks) using custom-written scripts that included components of the ImFluo toolbox (A.Esposito, E.N.I., Göttingen). In brief, donor images were binned $(2 \times 2)$ and subsequently subjected to low-pass Wiener and Gaussian filters with $3 \times 3$ kernel dimensions. Background signal was then removed by thresholding and FRET efficiencies calculated on a pixel-to-pixel basis by determining the difference between donor intensities before and after photobleaching of the acceptor and normalizing this value to the donor intensity after photobleaching 1.4. Cumulative histograms of FRET efficiencies were obtained by pooling FRET efficiency frequencies data for each experimental condi- 
tion ("+Ca ${ }^{2+"}$, "-Ca ${ }^{2+}$ ", "apoCaM", ...) and normalizing these frequencies to the total amount of pixels accounted by each curve.

\subsubsection{Overlay assays}

GST-fusion proteins of the C- and N-termini of EAG were induced with $1 \mathrm{mM}$ IPTG in BL21 (DE3) E. Coli (Stratagene) and harvested after 4 hrs induction, lysed by short sonication and boiled for $5 \mathrm{~min}$ in Laemmli's loading buffer. Proteins were separated by SDS-PAGE and either stained with Coomassie brilliant dye or transferred onto nitrocellulose membranes (Amersham). The membranes were blocked overnight at $4^{\circ} \mathrm{C}$ with $5 \%(\mathrm{w} / \mathrm{v})$ milk powder in TBS-T, followed by 2 hrs incubation at room temperature (RT) with $0.5 \mu \mathrm{g} / \mathrm{ml}$ biotinylated CaM (Calbiochem) and $1 \mathrm{mM} \mathrm{CaCl}_{2}$ in TBS-T, washed once for $10 \mathrm{~min}$ in $0.5 \%$ Triton TX-100, $1 \mathrm{mM} \mathrm{CaCl}_{2}$ in TBS-T and twice for $10 \mathrm{~min}$ in $1 \mathrm{mM} \mathrm{CaCl}_{2}$ in TBS-T. Membranes were then incubated for $1 \mathrm{hr}$ with $0.2 \mu \mathrm{g} / \mathrm{ml}$ HRP-conjugated Streptavidin (Pierce) in TBS-T with $1 \mathrm{mM} \mathrm{CaCl}$, washed four times for $5 \mathrm{~min}$ in the same buffer and detected using the Enhanced Chemo-Luminescence kit (ECL, Amersham).

\subsubsection{Generation of isoform specific antibodies against rEAG1 and rEAG2}

Selection of antigen peptides Rabbit polyclonal antibodies were generated against short (15-22 aa.), isoform specific peptide sequences of rEAG1 and rEAG2. The aminoacid sequences of both EAG isoforms were analyzed for potential epitopes. Both sequences were found to diverge mostly in their C-terminus cytosolic domains, therefore, five peptide sequences were selected in this region, based on potential antigenicity [34], solvent accessibility and degree of divergence from the complementary EAG isoform. Moreover, the uniqueness of these sequences in the rat proteome was verified using the BLAST program [1]. For rabbit immunization, epitope peptides were coupled to immunogenic-carrier proteins through cysteines. For this purpose, an extra cysteine was usually added to the N-terminus of each peptide. Peptide synthesis, rabbit immunization, animal care and bleeding were performed by BioGenes GmbH, Berlin. 


\begin{tabular}{llcc}
\hline \hline Epitope & Peptide \# & Sequence & Immunized Animals \\
\hline rEAG1 aa. $794-808$ & 49050 & CLGPKAGGGDPAKRK & $\# 7194, \# 7195$ \\
rEAG1 aa. $930-944$ & 49051 & ILMSRGSSQSPQDTC & $\# 7196, \# 7197$ \\
rEAG2 aa. $836-849$ & 49052 & C-SMGLLSEDPKGSDS & $\# 7198, \# 7199$ \\
rEAG2 aa. $941-954$ & 49053 & C-LLSEKSVPQTSSPK & $\# 7200, \# 7201$ \\
rEAG2 aa. $720-733$ & 49054 & C-RNQGSAQSDPERSQ & $\# 7202, \# 7203$ \\
rEAG2 aa. $967-988$ & 49055 & CQDIFSVSRPESPESDKDEINF & $\# 7204, \# 7205$ \\
\hline
\end{tabular}

Table 2.7: Antigen peptides used for polyclonal antibody production. "C-" denotes a cysteine introduced for coupling purposes.

Testing The antisera of immunized rabbits was tested for immunofluorescence applications on HEK293 cells transfected with rEAG1-GFP or rEAG2-GFP and fixed with $4 \%$ formaldehyde. Cells were stained (see §2.2.8) with serum dilutions of 1:5000, 1:1000, 1:500 and 1:100. Antiserum \#7194 was able to recognize cells transfected with rEAG1-GFP with a low background at a 1:5000 dilution. Similarly, antiserum \#7205 was able to recognize rEAG2-GFP transfected cells with a very low background when used in a 1:5000 dilution. These two antisera were isoform specific, as they did not recognize cells transfected with another EAG isoform. Moreover, no immunofluorescent signal was observed when the pre-immunization sera of both animals were used, further confirming the ocurrance of an immune-response against the injected peptides.

Affinity purification Antisera \#7194 and \#7205 were purified by affinity chromatography using the SulfoLink kit (Pierce) according to the manufacturers instructions. In brief, 1.3-1.6 mg of antigen peptides 49050 and 49055, used for immunization of animals \#7194 and \#7205, respectively, were coupled to SulfoLink columns via sulfhydril groups. The antigen-bound columns were incubated with their respective antisera and washed. Purified antibodies were then eluted using a low pH $(2.5$ - 3.0) buffer, dialyzed and stored at $-20^{\circ} \mathrm{C}$ in a buffer containing $100 \mathrm{mM}$ TRIS pH 7.4, $150 \mathrm{mM} \mathrm{NaCl}, 1 \mathrm{mg} / \mathrm{ml} \mathrm{BSA}$ and $0.025 \% \mathrm{NaN}_{3}$.

\subsubsection{Primary culture of hippocampal neurons}

Neuronal cultures were prepared from the hippocampi of embryonic day 18 Wistar rats as described elsewhere [3], with a few modifications. Hippocampi were isolated, digested for $15 \mathrm{~min}$ at $37^{\circ}$ in a Trypsin solution and dispersed mechanically. Following a short centrifugation, cells were resuspended in Neurobasal neuronal culture medium (without Phenol Red) supplemented with B27, $0.5 \mathrm{mM}$ L-Glutamine and 
$5 \mathrm{ng} / \mathrm{ml} \mathrm{FGF} \mathrm{(all} \mathrm{from} \mathrm{Invitrogen),} \mathrm{counted,} \mathrm{and} \mathrm{plated} \mathrm{at} \mathrm{an} \mathrm{appropriate} \mathrm{density}$ on glass coverslips coated with poly-L-Lysine (Sigma) and Laminin (Sigma).

\section{Transfection of primary hippocampal neurons}

Primary neuronal cultures were transfected on the third day after plating (3 DIV) using the Lipofectamine2000 (Invitrogen) transfection reagent and according to the instructions of the manufacturer. A total amount of $2 \mu \mathrm{g} c D N A$ and $3 \mu \mathrm{l}$ Lipofectamine per $25 \mathrm{~mm}$ coverslip was used. For co-transfections cDNA ratios of 3:1 to 10:1 (m/m) were used (EAG (donor):CaM (acceptor)).

\subsubsection{Immunofluorescence}

For immunostainings, cells were briefly washed with ice-cold PBS and fixed for 30 min with $4 \%$ formaldehyde (FA) at RT. Residual FA was quenched with $100 \mathrm{mM}$ Glycine in PBS for $5 \mathrm{~min}$, and cells were permeabilised for 5 min with $0.1 \%$ Triton X-100 in PBS and blocked with $0.2 \%$ Gelatine in PBS for 10 min. Subsequently, cells were incubated with the primary antibodies at appropriate dilution (see Table 2.3) for $45 \mathrm{~min}$ at RT, washed four times for $5 \mathrm{~min}$ with incubation buffer and incubated with fluorophore-conjugated secondary antibodies. Next, cells were washed four times for $5 \mathrm{~min}$ with incubation buffer, once with PBS for $10 \mathrm{~min}$ and once, briefly, with destilled water before mounting in ProLongGold antifade reagent (Invitrogen). 


\section{Results}

\subsection{Photoconversion product of YFP interferes with acceptor photobleaching measurement of FRET between Cyan and Yellow Fluores- cent Proteins}

The Cerulean/YFP FRET pair [46,61] was used in an intermolecular FRET assay for the binding of CaM to EAG1. FRET was measured by acceptor photobleaching in HEK293 cells co-transfected with Cerulean-fused hEAG1 and YFP-CaM. Cerulean was excited using $405 \mathrm{~nm}$ or $458 \mathrm{~nm}$ laser light, whereas YFP was imaged and bleached using the $514 \mathrm{~nm}$ line of an Ar laser. The differences in donor intensity before and after photo-destruction of the acceptor fluorophore in a region of interest were calculated on a pixel to pixel basis and normalized to the post-bleach donor intensity in order to determine FRET efficiency values. FRET efficiencies for each pixel are displayed in a color-coded image and plotted in a frequency histogram (Fig. $3.2)$.

FRET efficiencies measured using $405 \mathrm{~nm}$ excitation were consistently high in the nucleus of cells expressing high levels of YFP-CaM (Fig. 3.2 B) However, hEAG1 was not expressed in the nucleus and areas with low intensities of the donor also revealed high FRET efficiencies. Furthermore, cellular regions which were not bleached, showed no significant changes in donor intensity, thereby excluding any artifacts due to movement of the sample or laser power instability.

In order to test if the increase in the donor intensity was due to genuine FRET, occurring in an area with a low population of donor molecules, or to an artifact of Cerulean or YFP, the acceptor photobleaching protocol was repeated in cells transfected with hEAG1-Cerulean or YFP-CaM on their own (data not shown). 


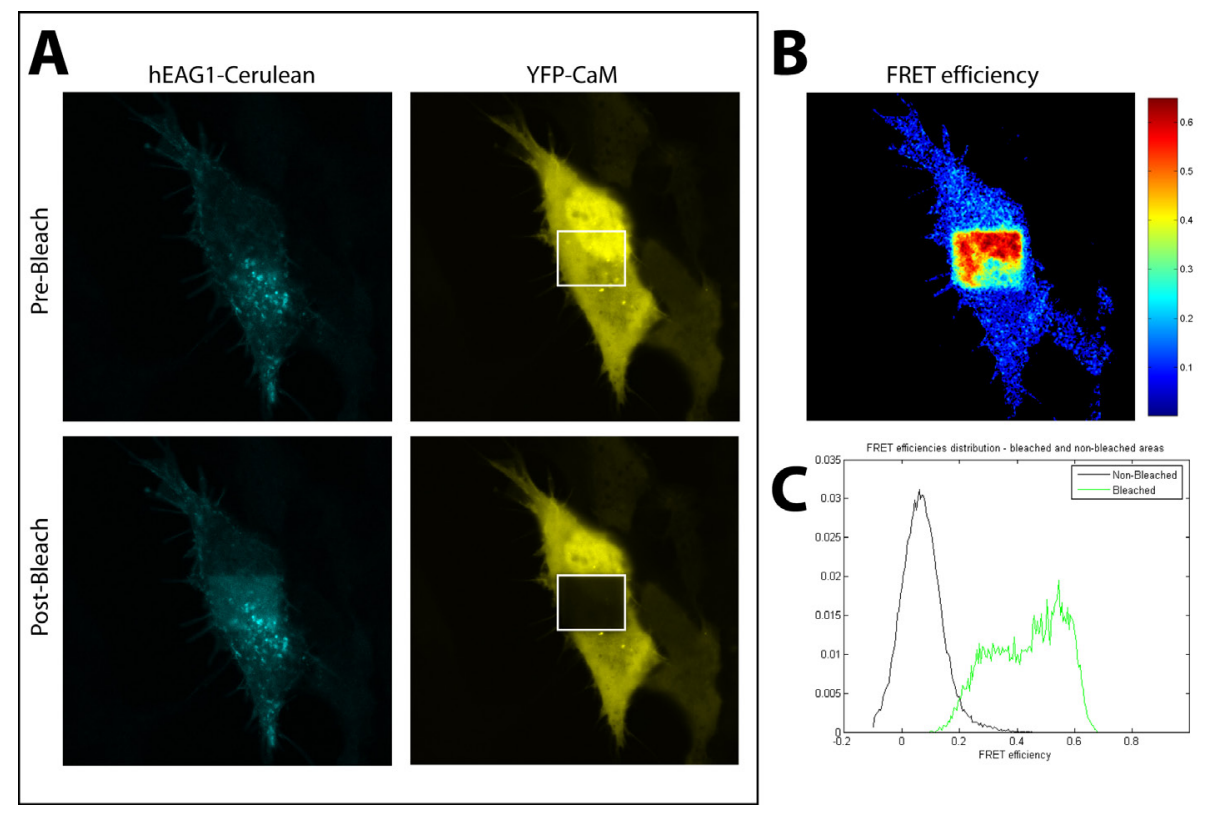

Figure 3.2: Photoconversion of YFP in HEK cells co-transfected with hEAG1-Cerulean and YFP-CaM. (A) Fluorescence emission in 465-505 $\mathrm{nm}$ range (CFP/Cerulean) with $405 \mathrm{~nm}$ excitation and in the 525-610 nm range (YFP) with $514 \mathrm{~nm}$ excitation, before and after photobleaching of YFP in a portion of the cell (white box) by continuous scanning with the $514 \mathrm{~nm}$ laser line. (B) FRET efficiency map. (C) Corresponding normalized cumulative histogram of FRET efficiencies. 


\begin{tabular}{|l|l|l|}
\hline exc. $458 \mathrm{~nm}$ & exc.405 nm & exc. $514 \mathrm{~nm}$ \\
\hline
\end{tabular}
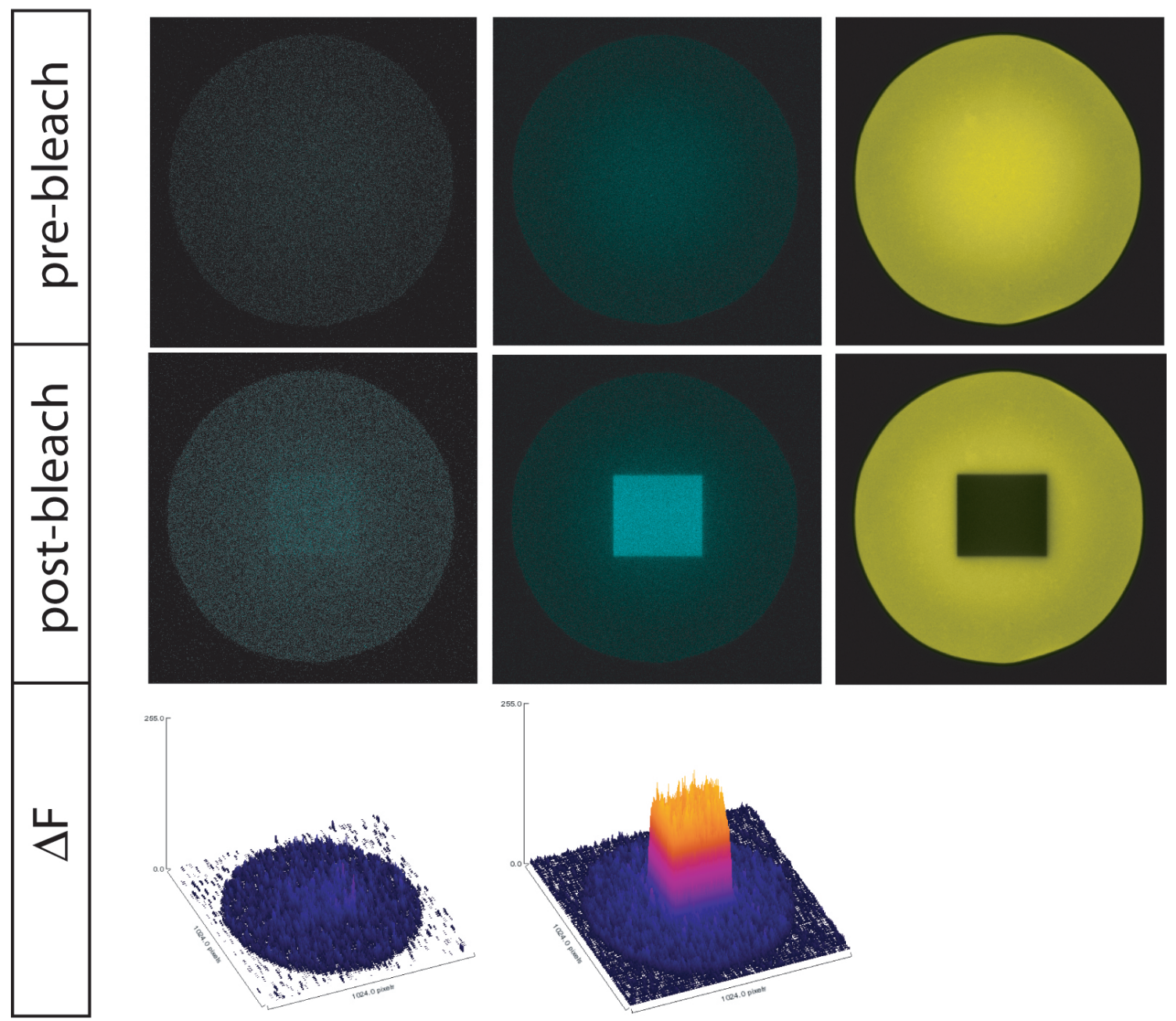

Figure 3.3: Excitation of the photoconversion product of YFP is more effective at 405 $\mathrm{nm}$ than at $458 \mathrm{~nm}$. Sepharose beads covalently coupled to purified YFP. Upper row: Fluorescence emission in 465-505 $\mathrm{nm}$ range (CFP/Cerulean) with 405 and $458 \mathrm{~nm}$ excitation and in the 525-610 $\mathrm{nm}$ range (YFP) with $514 \mathrm{~nm}$ excitation. Middle row: Emission of CFP and YFP channels after photobleaching of YFP in a specific portion of the bead by continuous scanning with the 514 laser line. Lower row: Colour-coded difference image $\left(\Delta F_{\text {donor }}=\right.$ Donor $_{\text {Post }}-$ Donor $\left._{\text {Pre }}\right)$ showing increase in fluorescence intensity in the 465-505 nm spectral range. 
Cells expressing only hEAG1-Cerulean showed no increase in intensity upon irradiation with $514 \mathrm{~nm}$ light whereas cells expressing YFP-CaM showed a large increase in intensity in the donor channel (465-505 nm) after photobleaching of YFP and upon excitation with $405 \mathrm{~nm}$. A smaller, but nevertheless significant increase was seen when $458 \mathrm{~nm}$ excitation was used. This intensity increase was limited to areas where YFP was bleached with $514 \mathrm{~nm}$ light. As no donor is present, this indicates that YFP could be photochemically converted to another fluorescent species (henceforth denoted XFP). In order to further characterize this photochemical reaction the bleaching procedure was performed in transfected live cells, fixed cells embedded in anti-oxidant medium (ProLongGold, Invitrogen) and in cells transfected with YFP on its own or Venus-CaM [51]. In all cases the bleaching of the yellow fluorophore resulted in the formation of XFP, which had significant emission in the spectral region of CFP/Cerulean (465-505 nm) when 405 or $458 \mathrm{~nm}$ excitation was used.

In order to exclude that cellular components are catalysing the photoconversion, YFP-coupled sepharose microbeads (a gift of Dr. F. Wouters) were mounted in Mowiol and partially bleached using the $514 \mathrm{~nm}$ laser line. Again, the bleaching of YFP led to a substantial increase in the fluorescence in the 465-505 $\mathrm{nm}$ range upon $405 \mathrm{~nm}$ excitation, and to a smaller increase when $458 \mathrm{~nm}$ excitation was used (Fig. 3.3). A spectral scan of the bleached regions in the range 415-640 $\mathrm{nm}$, acquired using $405 \mathrm{~nm}$ excitation (Fig. 3.4) revealed an emission peak at $\sim 470 \mathrm{~nm}$ for the XFP photoconversion product, i.e. overlapping with the $\mathrm{CFP} /$ Cerulean emission peak at $476 \mathrm{~nm}$.

The effect of the XFP photoconversion product on FRET efficiencies measured by acceptor photobleaching depends mostly on the relative concentrations of donor and acceptor. It is therefore of special importance for intermolecular biosensors in which donor and acceptor flurophores are fused to different constructs. Higher concentrations of YFP will result in the formation of larger amounts of XFP whose impact on FRET efficiencies will depend on the relative intensity of CFP/Cerulean donor molecules. This is due to the fact that increases in donor fluorescence $\left(\Delta F_{\text {donor }}\right)$ are normalized to the total donor intensity in each pixel in order to calculate FRET efficiencies (see Eq. 1.4). In areas such as the nucleus of cells transfected with hEAG1 even small intensity changes due to the photoconversion of YFP will result in large apparent FRET efficiencies. The use of $458 \mathrm{~nm}$ excitation instead of $405 \mathrm{~nm}$ can therefore reduce the effect of YFP photoconversion on FRET efficiencies, since it is much less efficient at exciting XFP than $405 \mathrm{~nm}$. 

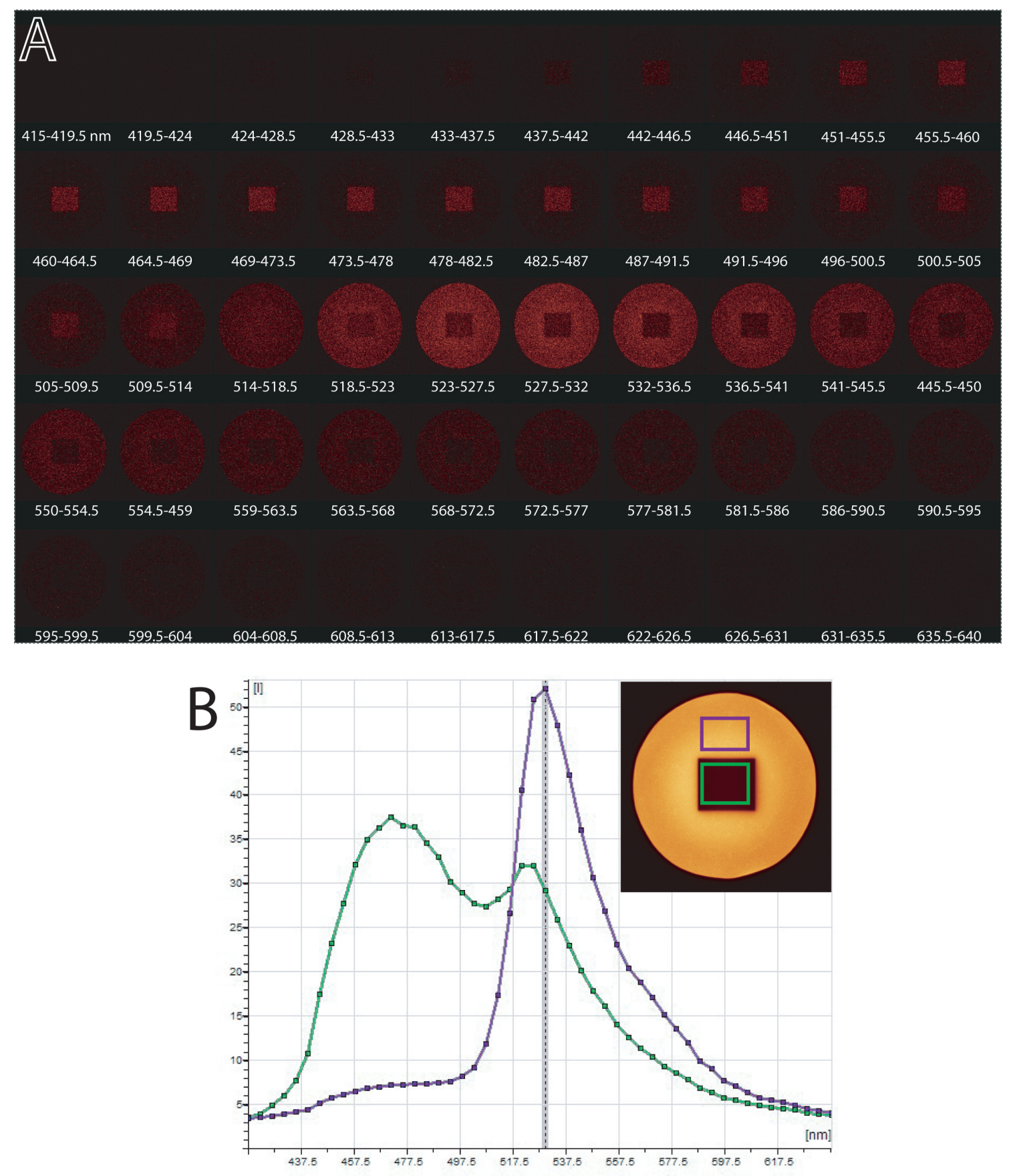

Figure 3.4: Spectral scan $(415-640 \mathrm{~nm})$ of the emission of the YFP photoconversion product. Sepharose bead covalently coupled to purified YFP. Middle square was bleached by continuous scanning at $514 \mathrm{~nm}$. (A) XFP emission under $405 \mathrm{~nm}$ excitation, one image was taken for every $4.5 \mathrm{~nm}$ portion of the spectrum. (B) Plot of fluorescence intensities for bleached (green) and unbleached (blue) regions of bead. 
For the experiments described in this work, Cerulean was imaged using $458 \mathrm{~nm}$ excitation light and regions of low donor expression, such as the nucleus, were thresholded. No effects of YFP photoconversion were detected after these two measures were applied. This was verified by using a biological control: acceptor photobleaching measurements of FRET between hEAG1-Cerulean and YFP-apoCaM - a mutated form of $\mathrm{CaM}$ which does not bind $\mathrm{Ca}^{2+}$ and, therefore, does not interact with the CaM binding domain of EAG1 (Fig. 3.5 F). No FRET was measured between both constructs, confirming the absence of photoconversion artifacts.

\title{
3.2 FRET assay for the $\mathrm{Ca}^{2+}$-dependent interac- tion of EAG with CaM
}

\author{
Expression of fluorescent-fusion constructs of EAG1 and CaM in HEK \\ cells
}

In order to establish a FRET assay for the $\mathrm{Ca}^{2+} / \mathrm{CaM}$-mediated regulation of EAG1 channels, channel subunits were labeled with fluorescent proteins at the C-terminus and CaM at the N-terminus.

When hEAG1 was fused at the C-terminus with EGFP and expressed in different mammalian cell lines, its fluorescence signal was located mostly in small vesicles in the cytoplasm and in the endoplasmatic reticulum (ER) / nuclear envelope (data not shown). Occasionally a membrane staining was discernible as a faint contour line. This expression pattern was the same as that of non-labeled overexpressed (pTracerheag1) channels (G. Bunt, personal communication) as detected by a monoclonal antibody (anti-33) recognizing an epitope in the intracellular part of the channel. The currents and kinetics of GFP-fused hEAG1 were furthermore indistinguishable from those of non-labeled channels (F. Monje, data not shown). This indicates that C-terminal fusion of the channel subunits to fluorescent proteins does not largely interfere with the localization and functionality of the channel.

For FRET experiments, the EGFP fused to EAG1 was exchanged by either the donor or acceptor of the EGFP-derived FRET pair Cerulean-YFP. Optimization experiments with the cytosolic C-terminus of rEAG1 showed that FRET could only be measured when EAG1 was labeled with the donor fluorophore Cerulean and CaM with the acceptor YFP, and not vice-versa. This configuration was therefore used 
throughout this work.

In order to characterize the interaction of EAG1 with CaM by FRET, the Ceruleanfused cytosolic C-terminus of rEAG1 was initially co-expressed with YFP-CaM in HEK cells. The C-terminus of the channel was localized uniformly across the cytoplasm but absent from the nucleus, whereas CaM was present in both of these cellular compartments. Therefore, regions of interest for FRET measurements were selected in the cytoplasm. Furthermore, in this study Cerulean was excited using the $458 \mathrm{~nm}$ laser line to avoid photoconversion effects of YFP.
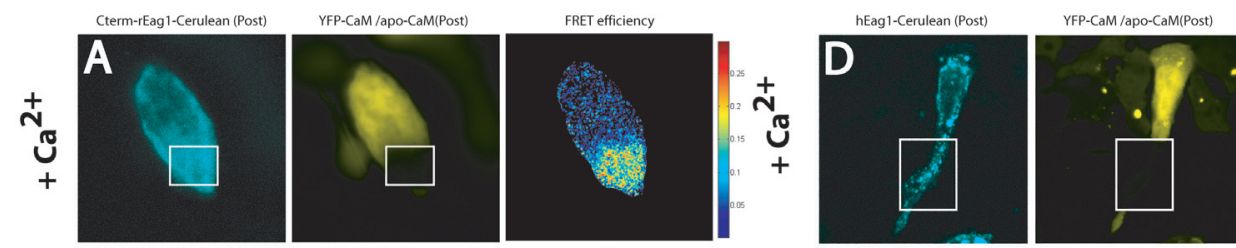

FRET efficiency
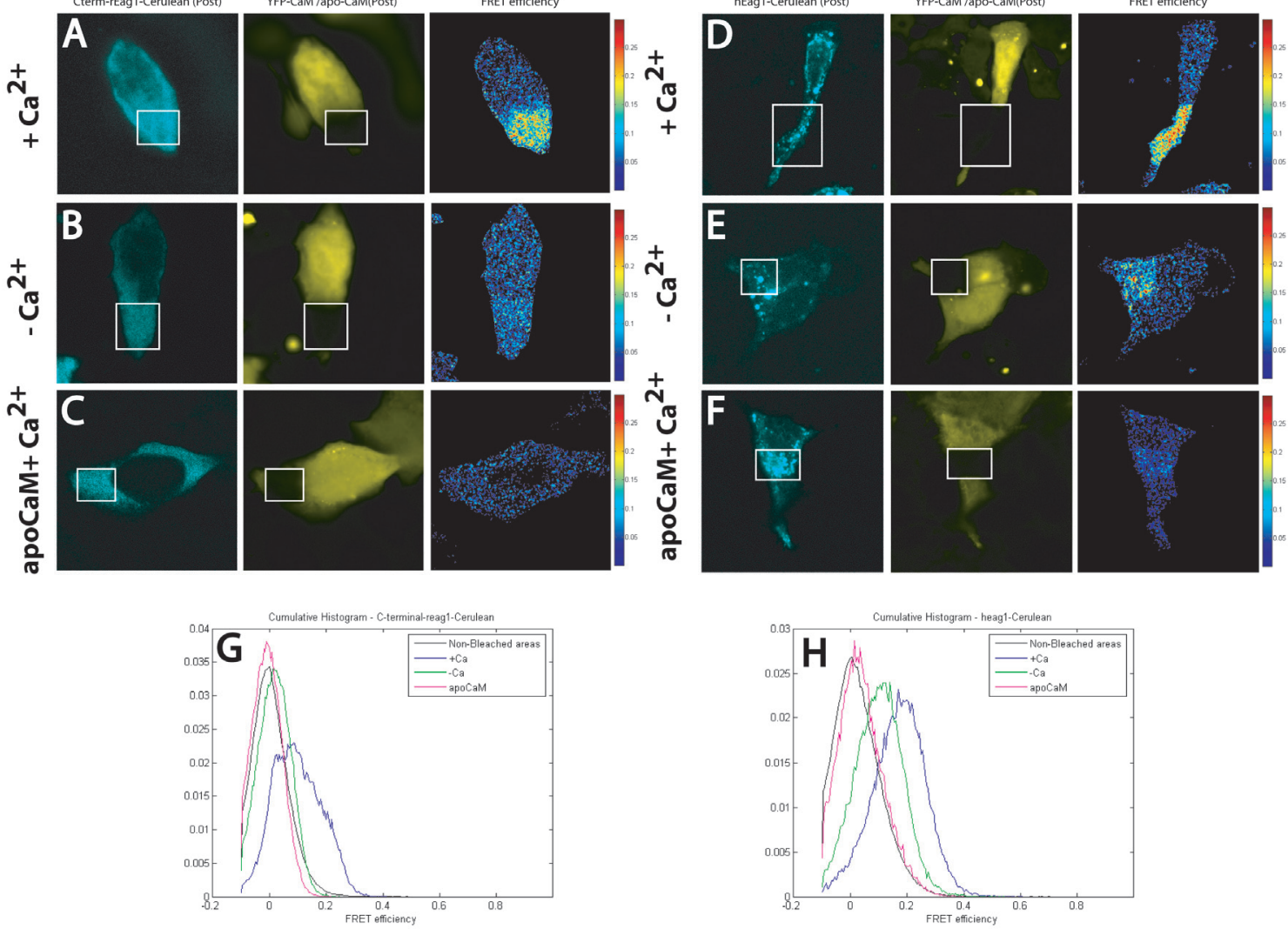

Figure 3.5: FRET measurements between EAG1-Cerulean (donor) and YFP-labeled CaM (acceptor), by acceptor photobleaching. HEK cells were co-transfected with either the cytosolic C-terminus of rEAG1 (A - C) or the full-length hEAG1 (D-F) labeled with Cerulean and YFP-labeled CaM constructs (A and D) and treated with $1 \mu \mathrm{M}$ ionomycin in the presence of $1.8 \mathrm{mM} \mathrm{Ca}^{2+}$; or $(\mathbf{B}$ and $\mathbf{E})$ with $1 \mu \mathrm{M}$ ionomycin in the presence of $1 \mathrm{mM}$ EGTA for $10 \mathrm{~min}$. (C-F) Co-transfection with apoCaM and stimulation with ionomycin in the presence of $1.8 \mathrm{mM} \mathrm{Ca}^{2+}$. Bleached areas are indicated by white boxes; Measured FRET is presented as efficiency map (colorscale maximum $=0.30$ ). Corresponding normalized cumulative histogram of FRET efficiencies of several cells $(n=11)$.

To investigate the $\mathrm{Ca}^{2+}$-dependency of the EAG1-CaM interaction, cells were placed in Ringer with either a high $\mathrm{Ca}^{2+}\left(1.8 \mathrm{mM} \mathrm{Ca}^{2+}\right)$ or a low $\mathrm{Ca}^{2+}$ concentration (no 
$\mathrm{Ca}^{2+}, 1 \mathrm{mM}$ EGTA) and stimulated with $1 \mu \mathrm{M}$ ionomycin, in order to artificially induce extreme intracellular $\mathrm{Ca}^{2+}$ conditions. Subsequently, cells were fixed and FRET was measured (Fig. 3.5).

No significant differences in the localization of the constructs was seen upon $\mathrm{Ca}^{2+}$ depletion or increase. However, in FRET experiments, the C-terminus of the channel was found to interact with $\mathrm{CaM}$ in a $\mathrm{Ca}^{2+}$-dependent manner. (Fig. $3.5 \mathrm{~A}, \mathrm{~B}, \mathrm{G}$ ). Cells with increased $\mathrm{Ca}^{2+}$ concentrations showed FRET efficiencies ranging up to $35 \%$, with an average of $9.8 \%$, whereas in cells with depleted $\mathrm{Ca}^{2+}$ no FRET could be measured. In the cumulative histogram of the pooled FRET efficiencies of several cells, this is seen as a shift of the FRET distribution curve to the left upon $\mathrm{Ca}^{2+}$ depletion, peaking around zero. This indicates that Cterm-rEAG1 binds CaM in its $\mathrm{Ca}^{2+}$-bound state only (Fig. $3.5 \mathrm{G}$ ). This is verified by the expression of the $\mathrm{Ca}^{2+}$-insensitive construct, YFP-apoCaM, as acceptor. The FRET distributions of apoCaM-transfected cells overlap those of $\mathrm{Ca}^{2+}$-depleted cells.

This $\mathrm{Ca}^{2+}$-dependent interaction with CaM was also found for the full-length hEAG1 channel. It displayed high FRET efficiencies with YFP-CaM, with an average of $17 \%$ and values ranging up to $40 \%$, in the presence of $\mathrm{Ca}^{2+}$ (Fig. $3.5 \mathrm{D}, \mathrm{H}$ ). In $\mathrm{Ca}^{2+}$-depleted cells, a reduced FRET-efficiency distribution with an average at $10 \%$ was obtained (Fig. 3.5 E, H). However, in cells where hEAG1-Cerulean was cotransfected with YFP-apoCaM, even in the presence of $\mathrm{Ca}^{2+}$, no FRET could be measured (Fig. 3.5 F, H). This indicates that the full-length channel is not capable of binding CaM in its $\mathrm{Ca}^{2+}$-unbound state. Consequently, it can be assumed that, even after depletion of $\mathrm{Ca}^{2+}$ in the presence of EGTA, there still are $\mathrm{Ca}^{2+}$-bound CaM molecules that account for the measured FRET efficiency. Moreover, the fact that no FRET was measured for the C-terminus under low $\mathrm{Ca}^{2+}$ conditions suggests that the full-length protein has a higher affinity for $\mathrm{Ca}^{2+}$ than the $\mathrm{C}$-terminus alone.

\section{Mutation of the CaM binding domain (F714S, F717S) of EAG1 prevents binding of CaM to the C-terminus but not to the full-length hEAG1 channel}

To understand the difference in $\mathrm{Ca}^{2+}$-dependent behaviour between the C-terminus and the full-length channel, EAG1 constructs carrying the mutations F714S, F717S in the C-terminal CaM-binding domain (CaMbd) were made $\left(\mathrm{Cterm}_{F 114 S, F 117 S^{-}}\right.$

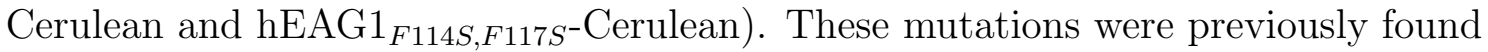
[70] to inhibit the binding of CaM to the C-terminus of hEAG1, resulting in $\mathrm{Ca}^{2+}$ - 
insensitive currents. The expression pattern of both mutation-containing constructs was not changed in comparison to their their wild-type counterparts. These constructs were co-expressed with YFP-CaM and subsequently exposed to extreme $\mathrm{Ca}^{2+}$ conditions prior to fixation.

The mutations F714S, F717S indeed inhibited the interaction of CaM with the Cterminus of rEAG1, since no FRET was measured under both the high and low $\mathrm{Ca}^{2+}$ conditions, as well as with apoCaM (Fig. 3.6 A-F).
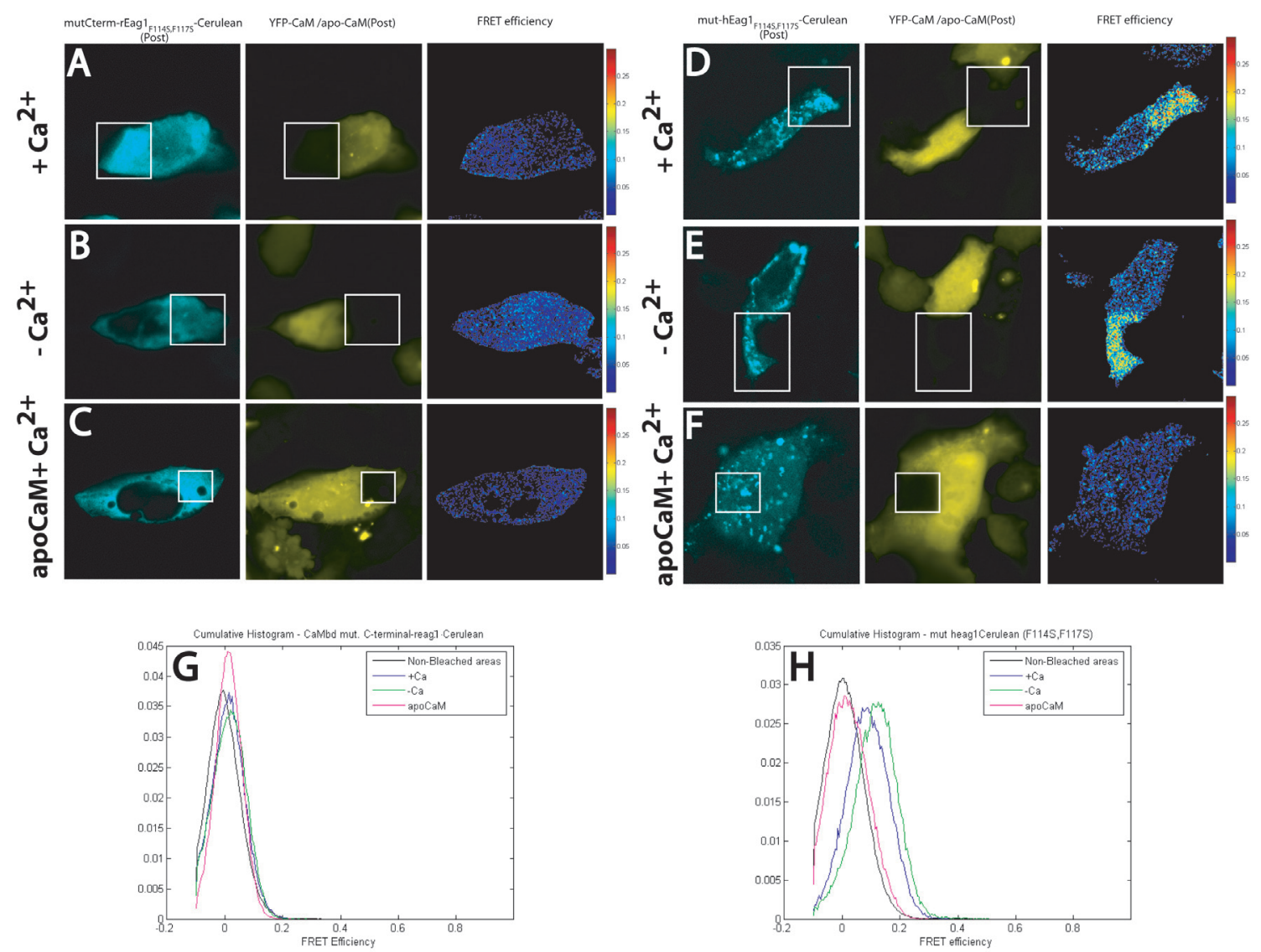

Figure 3.6: FRET measurements between EAG1-Cerulean with mutated CaMbd (F714S, F717S) and YFP-labeled CaM. HEK cells were co-transfected with the CaMbd-mutated cytosolic C-terminus of rEAG1 (A - C) or full-length hEAG1 (D-F) labeled with Cerulean and YFP-labeled CaM constructs. Cells were treated with $1 \mu \mathrm{M}$ ionomycin in the presence of $1.8 \mathrm{mM} \mathrm{Ca}^{2+}(\mathbf{A}, \mathbf{D}) ;(\mathbf{B}, \mathbf{E})$ or treated with $1 \mu \mathrm{M}$ ionomycin in the presence of 1mM EGTA. (C-F) Co-transfection of the EAG1 constructs with apoCaM and treatment ("+ $\mathrm{Ca}^{2+}$ ") with $1 \mu \mathrm{M}$ ionomycin for $10 \mathrm{~min}$. in the presence of $1.8 \mathrm{mM} \mathrm{Ca}^{2+} ;(\mathbf{G}, \mathbf{H})$ normalized cumulative histogram of FRET efficiencies of several cells $(n=11)$.

However, the results obtained with the full-length channel containing these CaMbd mutations show an interaction with YFP-CaM (with FRET efficiencies averaging around $10 \%$ and ranging up to $\sim 25 \%$ ) under both high $\mathrm{Ca}^{2+}$ conditions, 
as upon $\mathrm{Ca}^{2+}$ depletion. No significant differences in the FRET efficiencies of " $+\mathrm{Ca}^{2+"} /$ " $-\mathrm{Ca}^{2+"}$ cells were found. Furthermore no FRET was recorded between

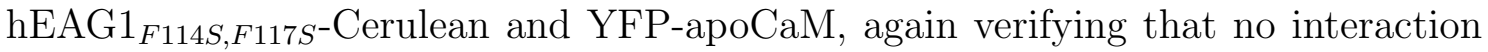
occurs with $\mathrm{Ca}^{2+}$-unbound CaM.

These results show that the previously reported [70] mutations in the C-terminal CaM binding site (F114S, F117S) of EAG1 indeed prevent the binding of CaM to the truncated, cytosolic C-terminus of the channel but not to the full-length protein. This implies that the full-length channel has either a higher affinity for CaM or can bind more CaM molecules than the C-terminus. Thereupon, it could be argued that the absence of measurable FRET for the mutated C-terminus is simply a consequence of a lowered affinity of the CaMbd. Mutations in the fulllength channel also lower the affinity, however not down to zero as the initial CaM affinity is higher for this construct, as a result of a more stable conformation of the full-length protein. However, the fact that the full-length construct does not display $\mathrm{Ca}^{2+}$-dependent changes in FRET efficiency favours the hypothesis of the existance of a second active binding site with a lower dissociation constant $\left(K_{d}\right)$, i.e. a higher $\mathrm{Ca}^{2+} / \mathrm{CaM}$ affinity, in the full-length channel. This putative site may be located in the cytosolic C-terminal tail - in which case it requires other parts of the channel subunit in order to be functional - or at another upstream location.
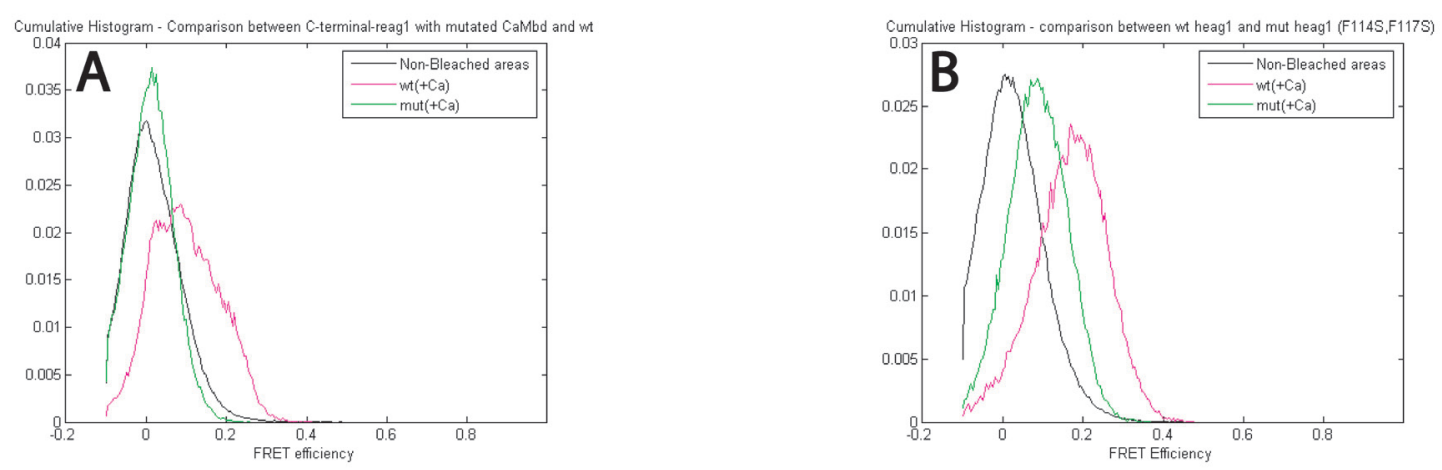

Figure 3.7: FRET measurements between the EAG1 and CaM. Normalized cumulative histograms comparing the obtained FRET efficiencies for CaMbd-mutated and wild-type C-terminus (A) and full-length EAG1 (B) constructs $(\mathrm{n}=11)$.

\subsubsection{CaM binds directly to the N-terminus of EAG1}

In order to screen for other potential CaM binding sites, in addition to the domain previously described in the C-terminus (aa. 707-726), the sequences of the intracellular tails of the channel were analyzed with a computational algorithm for CaM 
binding sites [94]. This suggested the existence of a binding site in the N-terminus at aa. 145-162 and two binding sites in the cytosolic C-terminus domain of hEAG1: the aa. 707-726 previously proposed by Schönherr et al. [70] and a second site at aa. 668-679 with an even higher CaM affinity.

Initially, the N-terminus of EAG1 was screened biochemically for the presence of CaM binding domains. The N-terminus cytosolic tail is, with 219 aa. , the second largest cytosolic segment of the channel subunit, after the C-terminus.

The cDNA coding for the cytosolic N-terminus domain of rEAG1 (aa. 1-219), as well a six shorter fragments (A-F see Fig. 3.8 A) were fused to GST in a bacterial expression vector and the proteins were expressed in BL21 (DE3) E. Coli. The bacterial lysates were separated on a 10\% SDS-Polyacrylamide gel (3.8 B) revealing the prominent bands of the expressed constructs. The GST-fused C-terminus and GST on its own were included as positive and negative controls, respectively.

A

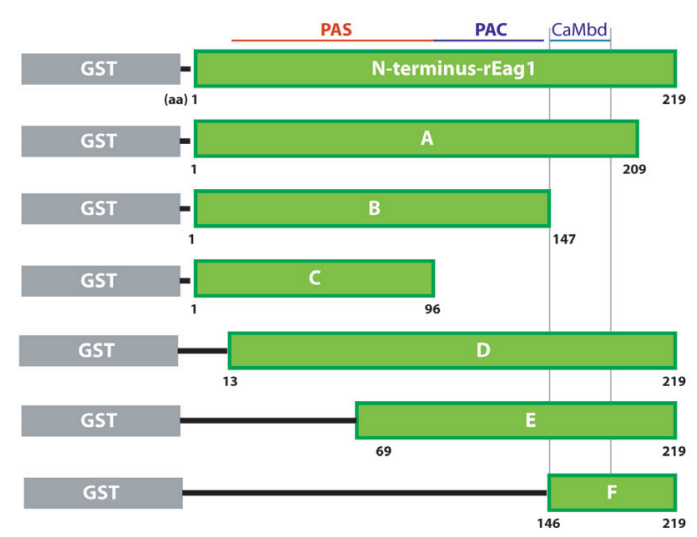

B

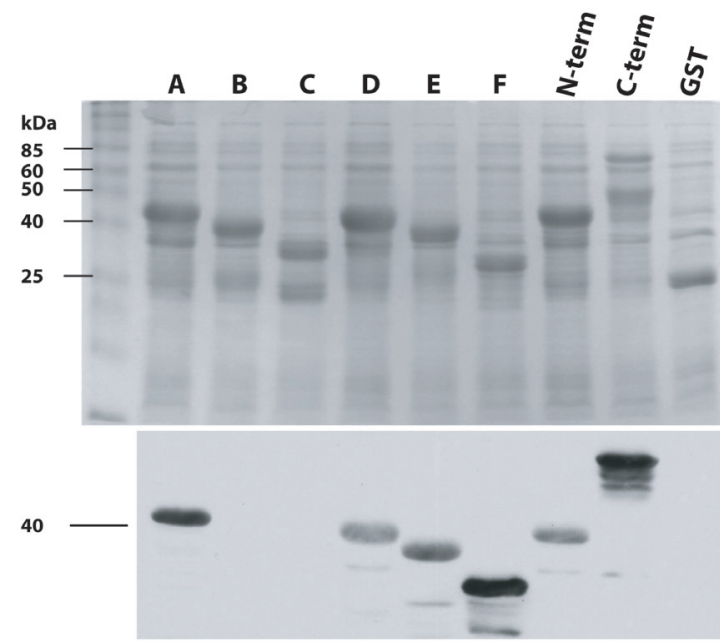

C

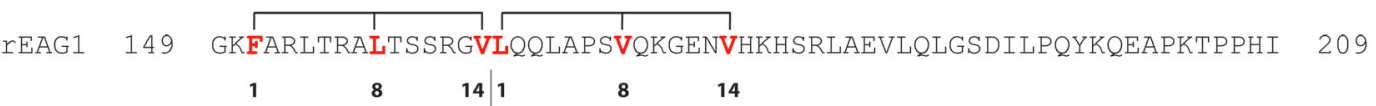

Figure 3.8: Calmodulin binds to segment aa. 147-209 of the N-terminus of rEAG1. Fragments of the N-terminus of rEAG1 were fused to GST (A) and bacterially expressed. (B) Coomassie-stained acrylamide gel loaded with bacterial lysates expressing fragments of the N-terminus and corresponding overlay blot (lower panel) incubated with biotinylated $\mathrm{CaM}$ in the presence of $1 \mathrm{mM} \mathrm{Ca}{ }^{2+}$. Detection with HRP-conjugated streptavidin yielded prominent bands for every fragment except $\mathrm{B}$ and $\mathrm{C}$, these segments do not contain aa. 147-209. (C) Amino acid sequence of the segment aa. 147-209 of rEAG1. The two putative 1-8-14 CaM binding motifs are highlighted.

The bacterial whole cell lysates were transferred onto a nitrocellulose membrane and incubated with biotinylated CaM. The resulting overlay blot showed binding of 
CaM to the N-terminal fragments with the exception of B and C. The GST-fused C-terminus, included as a positive control, also displayed a strong band, indicating a strong interaction with CaM. The fragments B and $\mathrm{C}$ do not contain the segment aa. 147-209, implying that a CaM binding domain may be present in this sequence. This is in accordance to the results of the computational screening.

Upon closer inspection of this sequence, two adjacent, putative $\mathrm{Ca}^{2+}$-dependent CaM-binding motifs of the 1-8-14 type were found at aa. 151-164 and 165-178 (3.8 C). Both sequences are located on a stretch which is predicted to be amphipathic and composed mostly of basic and hydrophobic residues. The second putative binding motif contains a proline, an aminoacid that seldom appears in amphipathic helices, which does not imply that this site cannot be involved in CaM binding.

\section{Mutations F151S, A152S prevent the binding of CaM to the N-terminus of the EAG1}

Mutations were introduced in the 1-8-14 motifs of the N-terminus of rEAG1 in order to localize the residues needed for the interaction with CaM. CaM binding sites of the 1-8-14 type are particularly sensitive to mutations in the first (Pos. 1) and last (Pos. 14) hydrophobic residues [60]. Three pairs of mutations were inserted in the GST fusion construct of the N-terminus.

The mutations L151S and A152S, were aimed at disrupting the hydrophobic region at the beginning of the first 1-8-14 motif at aa. 151-165. They should, in principle, affect this motif only and therefore provide information on whether both or only the first of the putative motifs actively binds CaM.

The middle mutations, V164S and L165S, were introduced with the intent of disrupting both adjacent 1-8-14 motifs in one construct. This was done by mutating the last hydrophobic amino-acid (V) of the first motif (aa. 151-164) and the first amino-acid (L) of the second motif (aa. 165-178). The hydrophobic amino-acids were replaced by serines, a residue of hydrophilic nature whose charge and size should minimise long range consequences of the substitutions. As a result of these mutations a 9 aa. long hydrophilic segment is created (aa. 159-168), which should hinder the formation of an amphipathic helix, a characteristic of functional 1-8-14 CaM-binding domains [60].

The third pair of mutations - V178S, H179D - target the last hydrophobic residue of the second putative 1-8-14 binding site (aa. 165-178), as well as an adjacent basic 
residue that was replaced by an acidic amino-acid.

The 3 constructs thus obtained were expressed in bacteria and tested biochemically for CaM binding on an overlay blot.

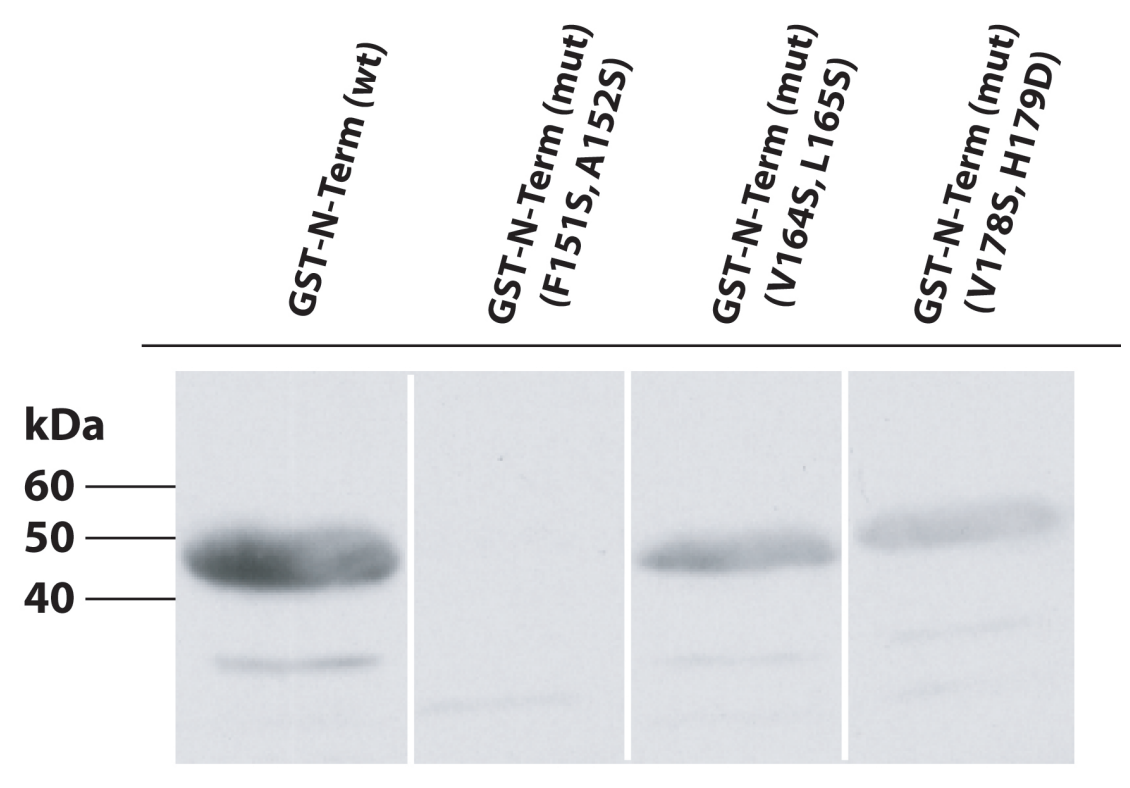

Figure 3.9: Mutations F151S, A152S disrupt the binding of CaM to the N-terminus of rEAG1. Overlay blot of the N-terminus of rEAG1 containing mutations in the region aa. 151-179. Detection with HRP-conjugated streptavidin revealed that mutations V164S, L165S and V178S,H179D caused a reduction in the binding of biotinylated CaM, whereas mutations F151S, A152S completely disrupted binding.

The overlay blot comparing the CaM binding of the wild-type GST-N-Term and the three mutants is shown in Fig. 3.9. Mutants V164S, L165S and V178S, H179D, involving the second 1-8-14 motif, display reduced binding to biotinylated CaM, in comparison to the wild-type GST-N-Term. However, only the mutation pair F151S, A152S, at the beginning of the first 1-8-14 motif, led to the disruption of binding.

These results show that the first 1-8-14 binding motif is the dominant CaM-binding domain in the N-terminus cytosolic segment of EAG1. 


\subsection{2 hEAG1 channels with mutated N-terminal (F151S, A152S) and C-terminal (F714S, F717S) CaMbd are unable to bind CaM}

The effect of the N-terminus CaMbd in the full-length channel was investigated by FRET microscopy. The mutations F151S, A152S were introduced in both wild-type hEAG1 and in a mutant with a disrupted C-terminal CaM-binding domain. FRET was measured by acceptor photobleaching in HEK293 cells under low and high Ca2+ conditions (Fig. 3.10).

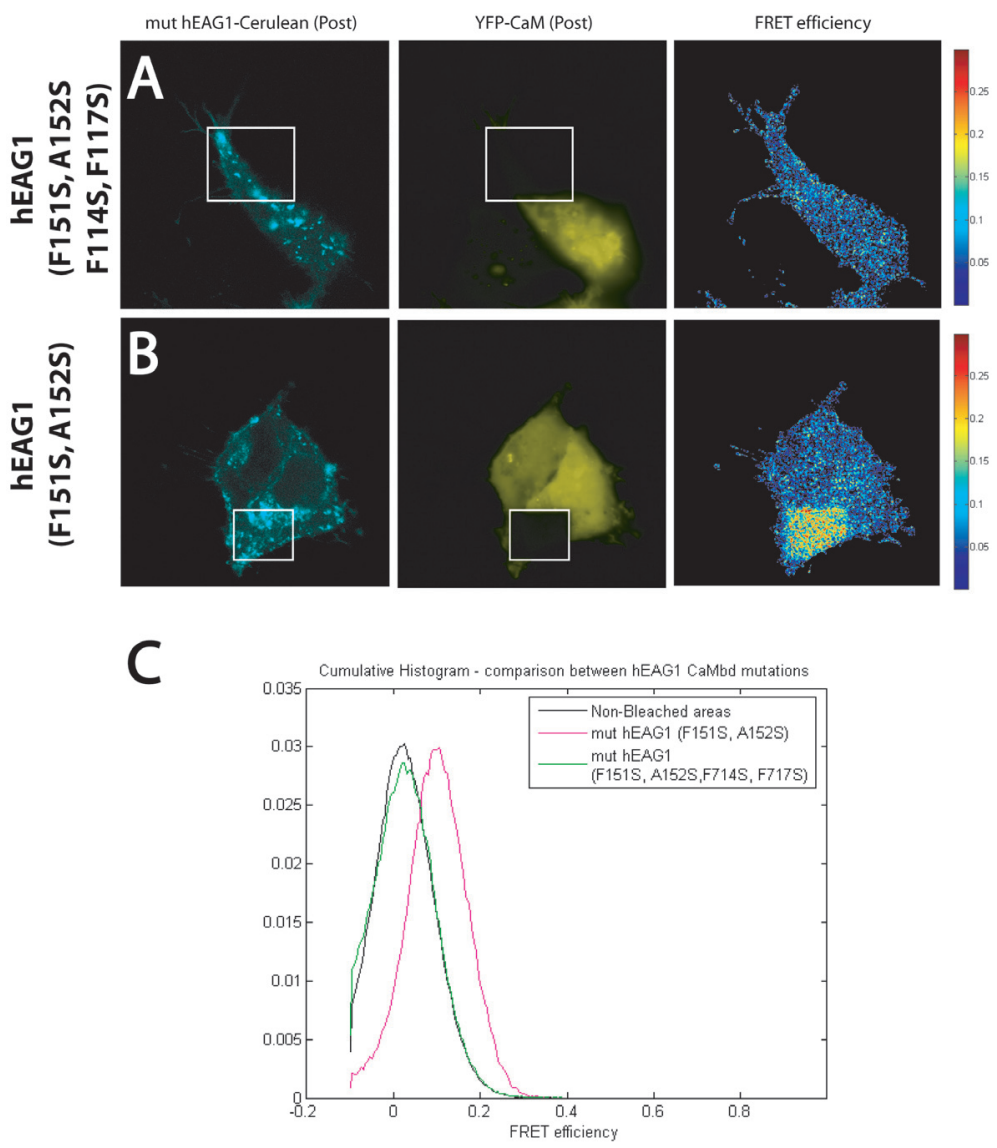

Figure 3.10: Mutations of the CaM binding domains in N- and C-termini completely disrupt the binding of CaM to hEAG1. Measurement of FRET by acceptor photobleaching in cells co-transfected with YFP-CaM and (A) hEAG1 $1_{\mathrm{F} 151 \mathrm{~S}, \mathrm{~A} 152 \mathrm{~S}, \mathrm{~F} 714 \mathrm{~S}, \mathrm{~F} 717 \mathrm{~S}}$ or (B) hEAG1 $1_{\mathrm{F} 151 \mathrm{~S}, \mathrm{~A} 152 \mathrm{~S}}$ after treatment with $1 \mu \mathrm{M}$ ionomycin in the presence of $1.8 \mathrm{mM} \mathrm{Ca}^{2+}$. (C) Mutations in the N-terminus CaM binding domain reduce but do not completely disrupt the binding of CaM to the channel - $c f$. Fig. $3.7(\mathrm{n}=10)$.

Constructs of hEAG1 containing both the mutated N- and C-terminal CaM binding domains were found not to interact with YFP-CaM, as no FRET could be measured. Fusion constructs of hEAG1 containing only the N-terminal mutations F151S and 
A152S interacted with YFP-CaM (revealing FRET average efficiencies of $~ 10 \%$ and ranging up to $30 \%$ ).

The combined four mutations F151S, A152S, F714S, F717S are therefore sufficient to hinder the binding of CaM to hEAG1. This implies that these two binding sites are responsible for CaM binding, although other binding sites can still be involved in the binding mechanism in a cooperative manner (e.g., the putative binding site found in silico). Even though no FRET interactions were detected between the truncated C-terminus with a mutated CaM binding domain (F114S, F117S) and YFP-CaM, there remains the possibility that a putative site at aa. 668-679 is functional but requires a full-length channel in order to bind CaM.

In conclusion the present results show that two CaM binding sites are present in hEAG1 subunits - a high-afinity CaM binding domain of the form 1-8-14 in the N-terminus and the previously described C-terminal binding domain at aa. 707276. Mutations at these sites lead to the complete disruption of CaM binding to the hEAG1, these two binding domains are therefore responsible for CaM binding. These findings indicate that the regulation of hEAG1 by $\mathrm{Ca}^{2+} / \mathrm{CaM}$ is more complex than previously thought.

\subsubsection{The EAG2 homologue interacts with $\mathrm{CaM}$ in a $\mathrm{Ca}^{2+}$ dependent manner}

Although EAG1 and its homologue EAG2 share a significant part of their aminoacid sequence (Fig. $3.11 \mathrm{E}$ ), the binding of EAG2 to CaM has so far not been described. In order to verify if EAG2 is interacting with CaM, FRET measurements were performed in cells co-transfected with a Cerulean-fusion construct of rEAG2 and YFP-CaM/EYFP-apoCaM (Fig. 3.11). Cerulean-labeled rEAG2 was located mostly in the endoplasmatic reticulum and Golgi network, with a few vesicles being visible in the cytosol. This localization was characteristic of the fluorescent fusion constructs of rat variants of EAG1 and EAG2, which displayed a higher tendency to remain in the secretory apparatus of cells.

rEAG2 was found to interact to interact with $\mathrm{CaM}$ in a $\mathrm{Ca}^{2+}$-dependent manner similar to hEAG1. The FRET efficiencies distribution peaked for high $\mathrm{Ca}^{2+}$ conditions with an average of $14 \%$. This indicates that a the mechanism of $\mathrm{Ca}^{2+} / \mathrm{CaM}$ binding is probably conserved in EAG isoforms, and not limited to EAG1. 


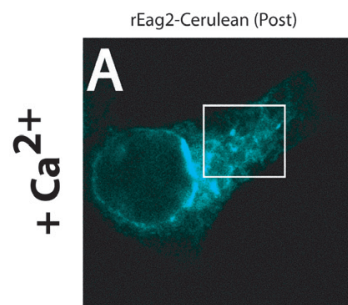

YFP-CaM /apo-CaM(Post)
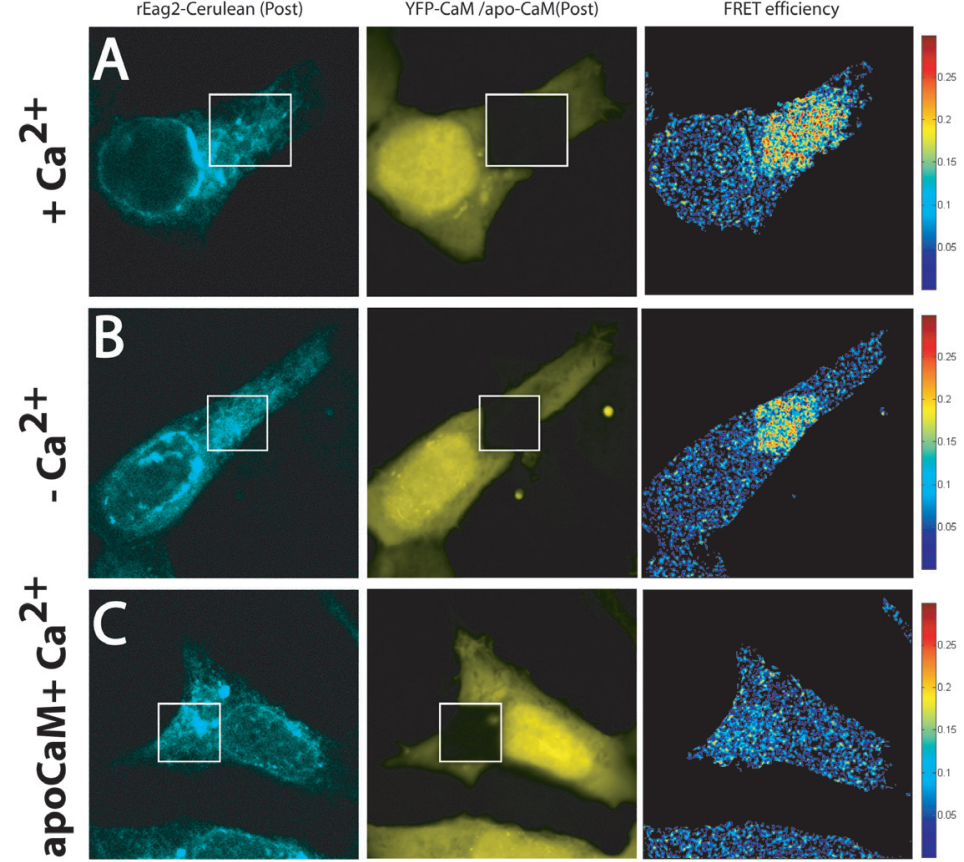

D

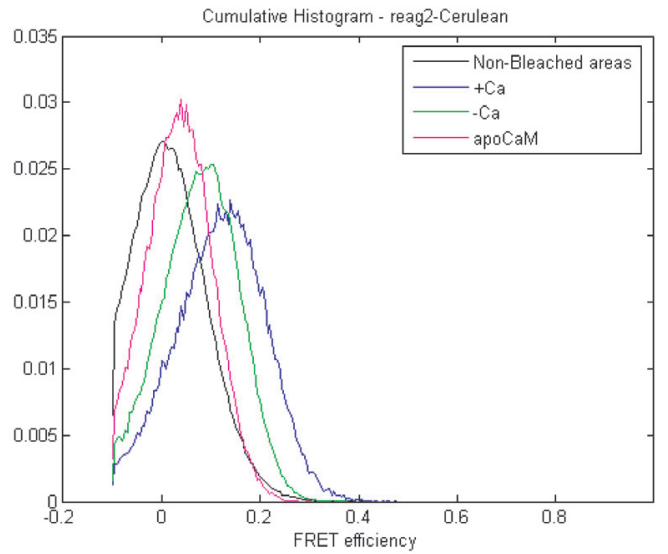

\begin{tabular}{|ccccc|}
\hline E & N-term CaMbd & & C-term CaMbd \\
147 & GWGKFARLTRALTSSRGVLQ & heag1 & DHPVRRLFQRFRQQKEAR-LA & 726 \\
147 & GWGKFARLTRALTSSRGVLQ & reag1 & DHPVRRLFQRFRQQKEAR-LA & 726 \\
145 & GWTKFARLTRALTNSRSVLQ $^{*}$ & reag2 & DHPVRKLFQKFKQQKELRNQG & 723 \\
& SS $^{*}$ & & $\mathrm{~S}^{*} \mathrm{~S}^{*}$ & \\
\hline
\end{tabular}

Figure 3.11: FRET measurements between rEAG2-Cerulean and YFP-labeled CaM. HEK cells were co-transfected with rEAG1 (A-C) labeled with Cerulean and YFP-labeled CaM constructs. Cells were treated with $1 \mu \mathrm{M}$ ionomycin in the presence of $1.8 \mathrm{mM}$ $\mathrm{Ca}^{2+}$ (A); or (B) treated with $1 \mu \mathrm{M}$ ionomycin in the presence of $1 \mathrm{mM}$ EGTA. (C) Cotransfection of $\mathrm{rEAG} 2$ with apoCaM and treatment $\left(+\mathrm{Ca}^{2+}\right)$ with $1 \mu \mathrm{M}$ ionomycin for 10 min in the presence of $1.8 \mathrm{mM} \mathrm{Ca}^{2+}$; (D)- Normalized cumulative histogram of FRET efficiencies of several cells $(n=10)$. (E) Sequence alignment of the $\mathrm{N}$ and C-terminal CaM binding domains of hEAG1, rEAG1 and rEAG2. The position of the CaMbd-disrupting serine substitutions $\left(\mathrm{S}^{*}\right)$ is conserved 


\subsection{Study of the localization and $\mathrm{Ca}^{2+} / \mathrm{CaM}$ regu- lation of EAG in rat hippocampal cultures}

EAG1 channels have been shown to affect neuronal excitability in Drosophila melanogaster, as eag mutants reveal a high rate of spontaneous activity $[8,21,31]$. This phenotype is compatible with the channel's activation threshold being close to the membrane resting potential. Therefore, the regulation of the channel by $\mathrm{Ca}^{2+} / \mathrm{CaM}$ can have a strong effect on neuronal activity. However, the physiological role of EAG1 as well as its regulation in neurons is not yet well understood. In order to gain insight in the spatio-temporal resolution of the EAG1/CaM interaction in the regulation of neuronal excitability, studies of the localization of EAG in neurons were performed and a FRET assay for CaM binding implemented in primary hippocampal cultures.

\section{Expression of rEAG1-Cerulean in rat hippocampal neuronal cul- tures}

rEAG1-Cerulean and YFP-CaM were cloned under the control of the human Synapsin promoter, and transfected in E18 dissociated hippocampal cultures. The synapsin-promotor restricts the expression of the channel to neurons [32]. This was verified by co-staining of the transfected cells with neuron-specific anti- $\beta$ tubulin III antibodies [17,76].

When transfected in neuronal cultures and at 12 days-in-vitro (DIV) rEAG1Cerulean had an expression pattern consisting mostly of small vesicular structures in the cytosol, which were present in soma, dendrites and axons. The latter was shown by co-staining with dendrite-specific MAP2 antibodies $[12,41]$, where rEAG1 was expressed in both MAP2-positive as well as negative neurites (Fig. 3.16). Additionally, rEAG1 transfected neurons showed no significant morphological differences when compared to untransfected cells. In order to verify if bursts of neuronal activity have an immediate effect on rEAG1 distribution, transfected cultures were stimulated with $90 \mathrm{mM} \mathrm{K}^{+}$for $180 \mathrm{~s}$. No differences in expression pattern resulted from this stimulation.

Endogenous EAG1 channels have been shown to be present at both RNA $[18,39,66]$ and protein level [25] in the hippocampus of adult rats, predominantly in the regions CA2, CA3, CA4, and DG, but also in CA1. However, the subcellular localization of 
EAG1 in these regions is not known.

\section{Subcellular localization of EAG channels in rat hippocampal neuronal cultures}

In order to investigate the intracellular localization of EAG channels 12 polyclonal antibodies were raised in rabbits against 6 isform-specific epitopes in the intracellular parts of the C-terminus of rEAG1 (2) and rEAG2 (4). The sequence of the C-terminus of the channel has several zones of high potential antigenicity and, moreover, contains less similarities between EAG1 and EAG2.

These antibodies were screened for use in immuno-fluorescence in formaldehydefixated HEK cells transfected with rEAG1-GFP or rEAG2-GFP fusion constructs. The anti-rEAG1 \#7194 (against aa. 794-808) and anti-rEAG2 \#7205 (aa. 967-988) antibodies were selected for their ability to recognize their targets with a low background while remaining specific for an EAG isoform (Fig. 3.12). The optimal final concentration was $\sim 3 \mu \mathrm{g} / \mathrm{ml}$ for both antibodies.

Hippocampal cultures of E18 rats at culture stages between 7 and 23 DIV were fixed with $4 \%$ formaldehyde and stained with anti-rEAG1 7194 and anti-rEAG2 7205 antibodies . Cultures stained with anti-rEAG1 antibodies at different concentrations displayed no detectable signal. However, the same antibody was capable of detecting overxpressed rEAG1-GFP, again with no signal being detected in adjacent untransfected cells (Fig. 3.13). This implies that the amounts of endogenous rEAG1 in hippocampus primary cultures with 7-23 DIV are indeed very low in comparison with the overexpressed channel. In contrast, in cultures stained with anti-rEAG2 antibodies a few cells with a punctated fluorescent signal were present along neuronal processes (both MAP2 positive and negative) and also in cells which were not co-stained with MAP2 at all (Fig. 3.14). Since MAP2 is a marker of the somatodendritic compartment of neurons $[12,41]$, these results may indicate that EAG2 is also present in non-neuronal cells of hippocampal primary cultures.

Endogenous rEAG2 also partially co-localized with p38, although, given the high density of the p38 staining some co-localization is always to be expected (Fig. $3.15)$.

There is no extensive literature on the subcellular localization of EAG channels. One article [25] describes the distribution of rEAG1 and rEAG2 in dissociated E18/E19 rat hippocampal cultures. In that study EAG1 was found to be expressed in the 


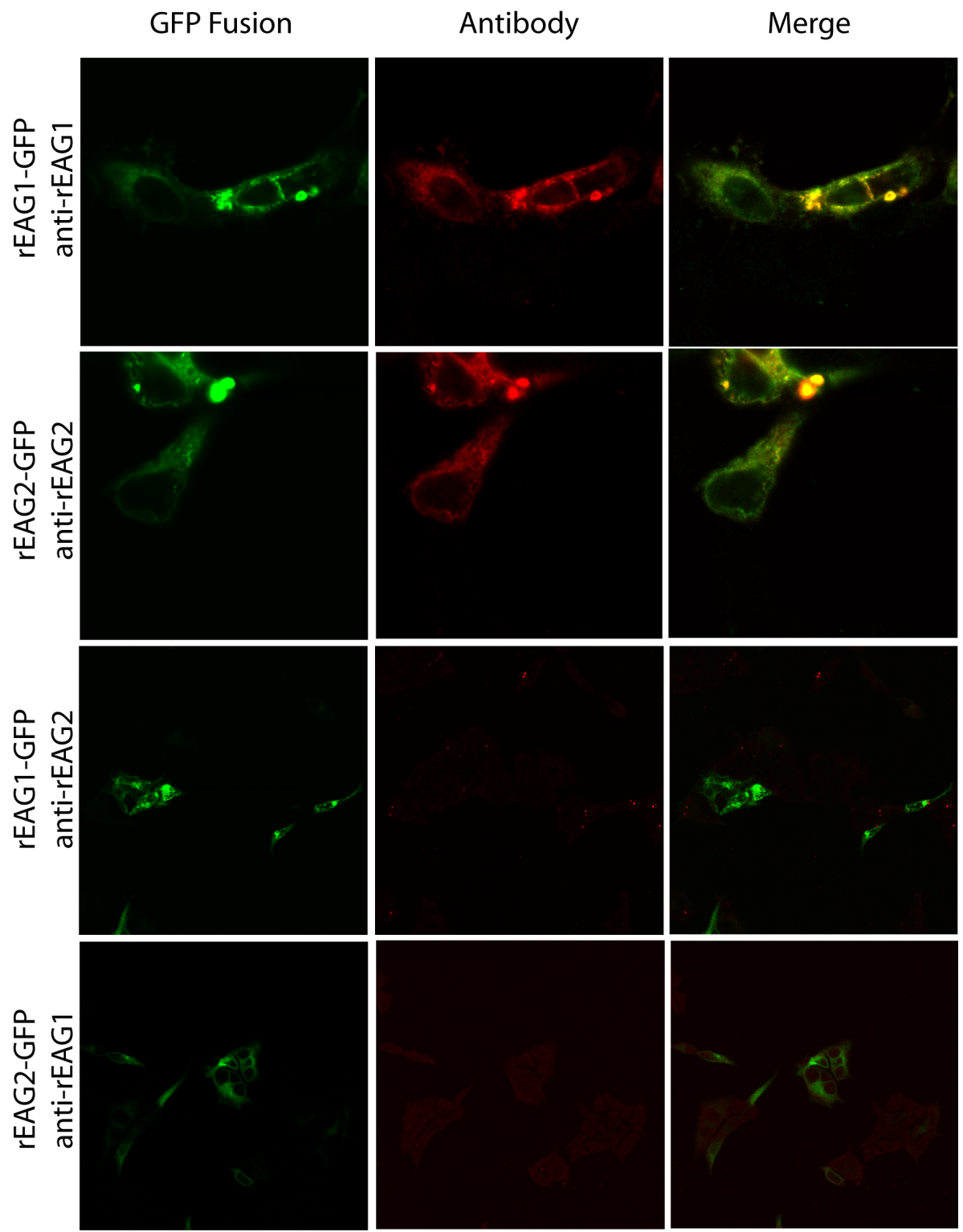

Figure 3.12: Tests for the use of anti-rEAG antibodies in immunofluorescence. HEK293 cells expressing GFP-fusion constructs of rEAG1 and rEAG2 (green) were fixed with 4\% formaldehyde and stained with \#7194 anti-rEAG1 and \#7205 anti-rEAG2 antibodies (red). Both antibodies were able to recognize their antigens specifically, with no crossreactivity for the other $\mathrm{rEAG}$ isoform (lower rows). The optimal final concentration for both antibodies was $\sim 3 \mu \mathrm{g} / \mathrm{ml}$. 
rEAG1-GFP

anti-rEAG1 (7194)
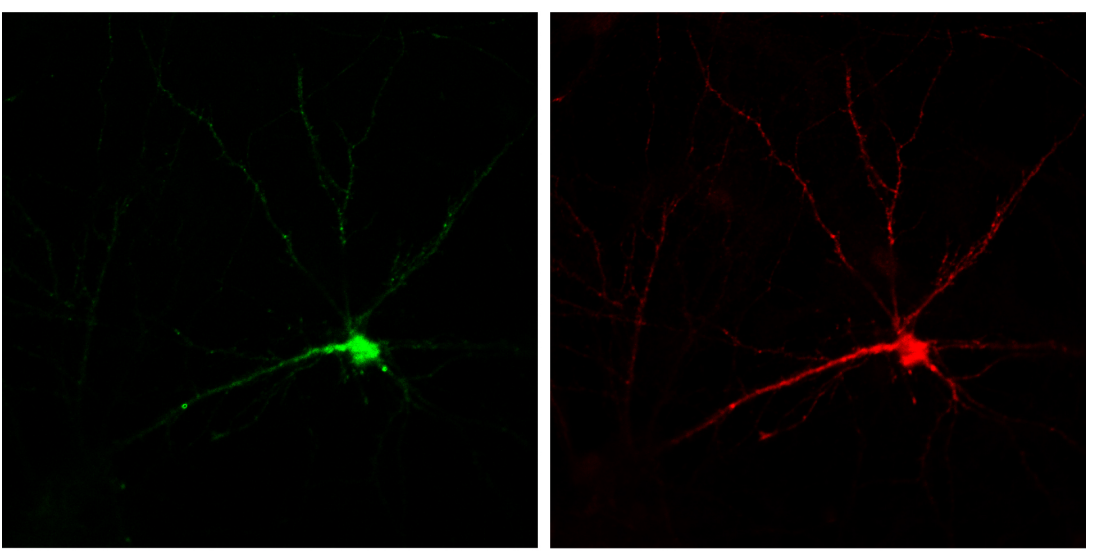

anti-p38

Merge
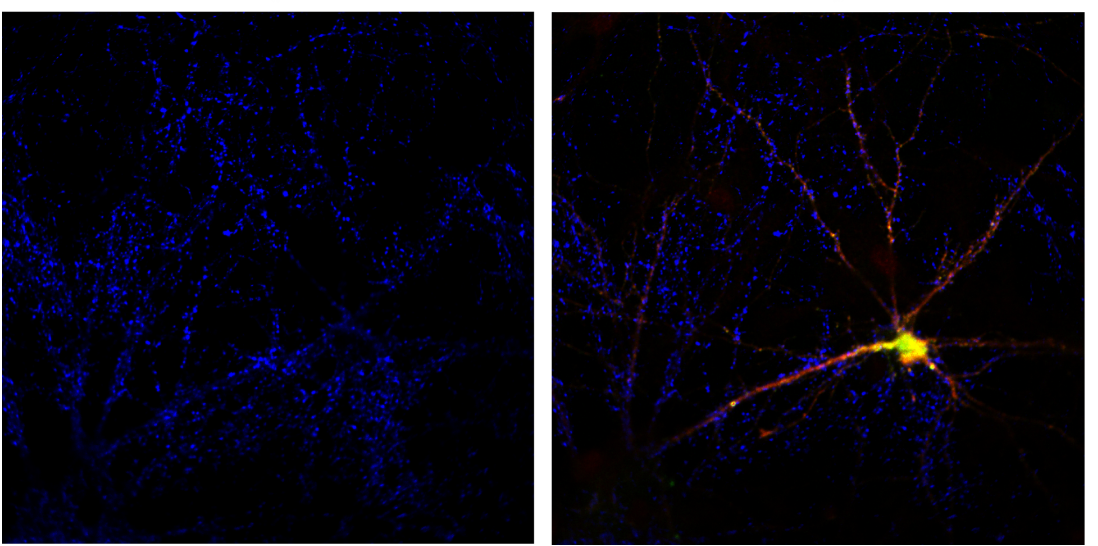

Figure 3.13: Immunostaining of rEAG2 in rat hippocampal cultures (E18, 12 DIV) transfected with (hSyn) rEAG1-GFP. Overexpressed rEAG1 (green) is detected by \#7194 antibody (red) whereas no endogenous signal is found in surrounding cells, which were stained with p38 antibody (blue). 


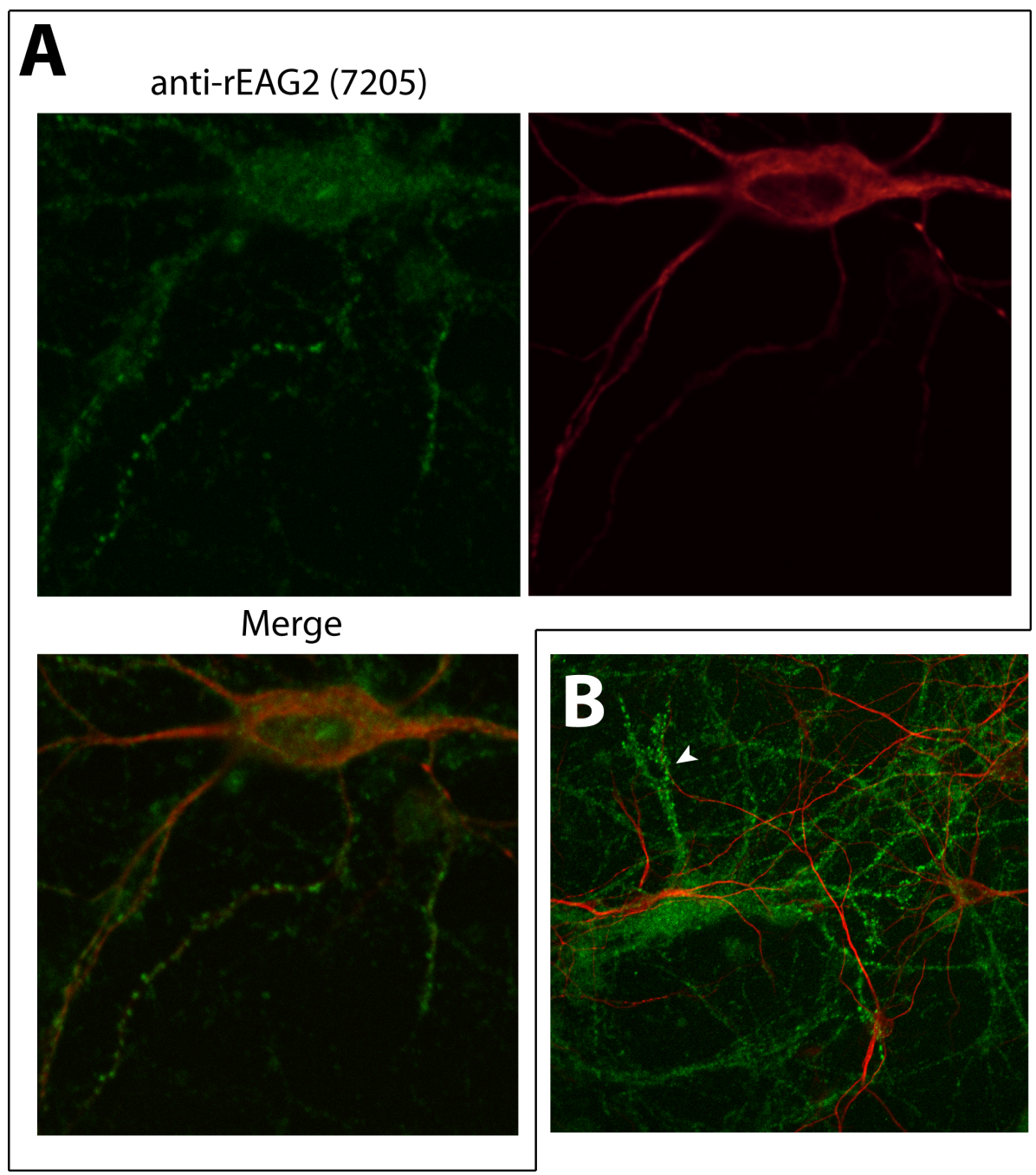

Figure 3.14: Immunostaining of rEAG2 in rat hippocampal cultures (E18, 20 DIV). (A) Endogenous rEAG2 (green) puncta are detected with \#7205 antibody in neurites stained with MAP2 (red) but also in MAP2 negative processes. (B) Endogenous rEAG2 puncta are also present in cell bodies that were not stained with MAP2 (arrowhead). 
anti-rEAG2 (7205)

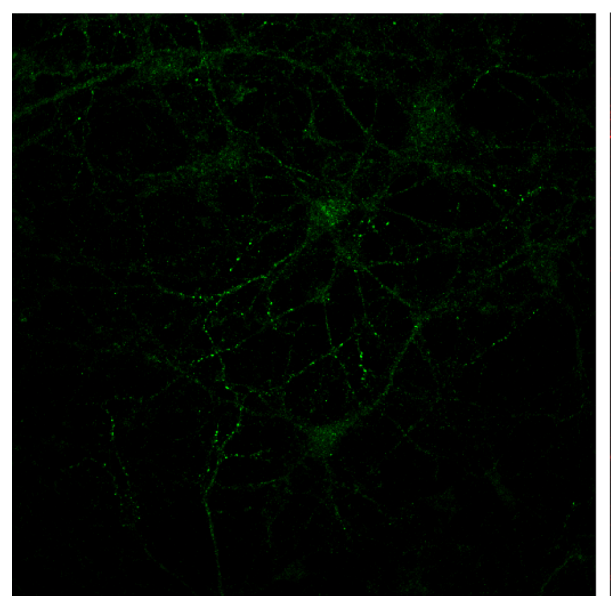

\section{Merge}

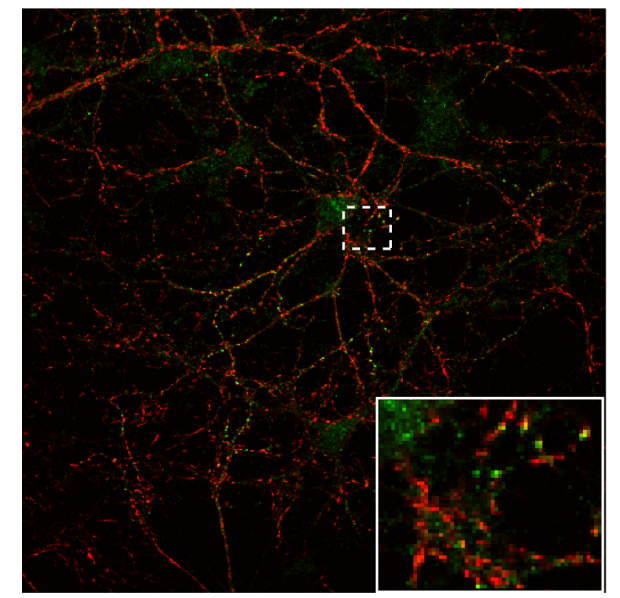

Figure 3.15: Rat hippocampal cultures (E18, 20 DIV) double stained for rEAG2 (\#7205 antibody, green) and p38 (red). Many p38 puncta do not colocalize with rEAG2, whereas most rEAG2 puncta do colocalize with p38.

anti-p38

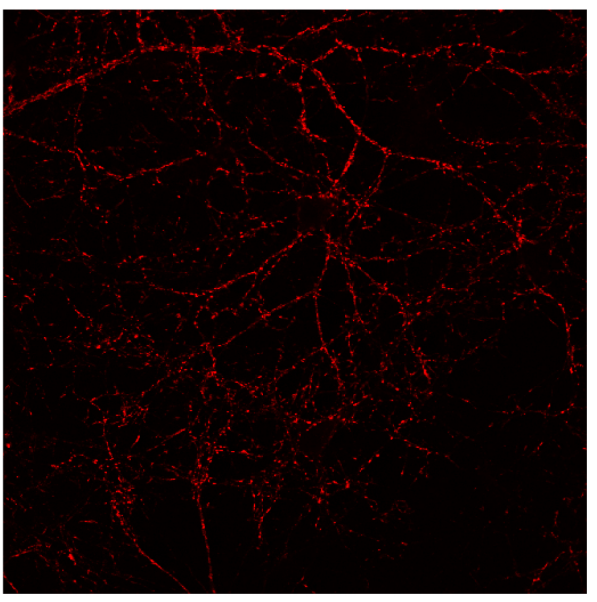

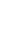


somatodendritic compartments of hippocampal neurons, in particular in the excitatory post-synaptic terminals since it co-localized with MAP2 positive processes as well as with p38 and Densin clusters (markers of chemical synapses and glutamatergic synapses, respectively). However, unpublished electron microscopy studies have found EAG1 to be present in the pre-synaptic terminals of rat hippocampal neurons (M. E. Rubio and L. Pardo, personal communication).

A co-staining of overexpressed rEAG1-GFP with p38 antibodies revealed little colocalization of EAG1 and p38 clusters. Both stainings are very dense making it very likely that some degree of co-localization occurs.

(hSyn) rEAG1-GFP
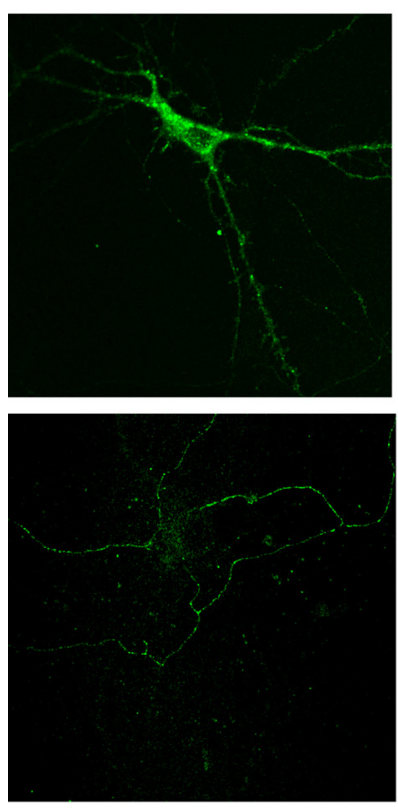

(hSyn) rEAG1-GFP

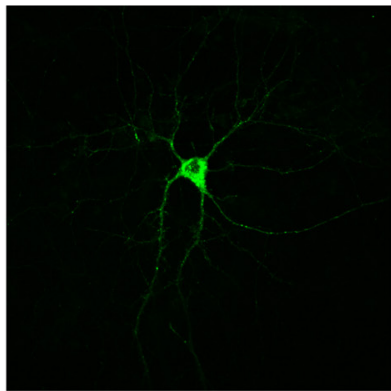

anti-MAP2 (sec.Cy5)
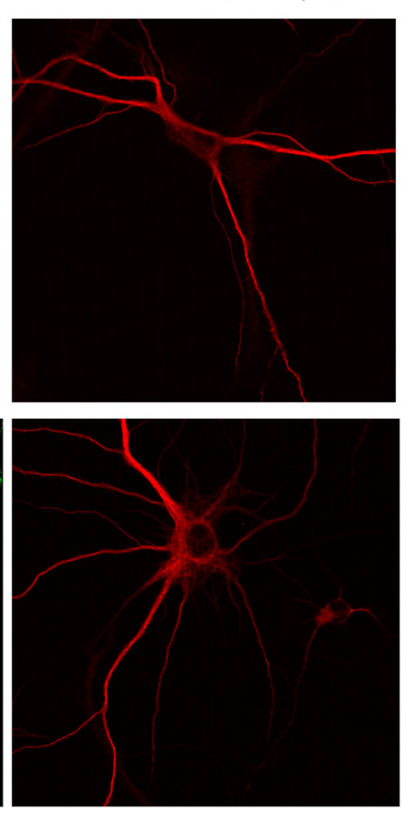

anti-p38 (sec. Cy5)

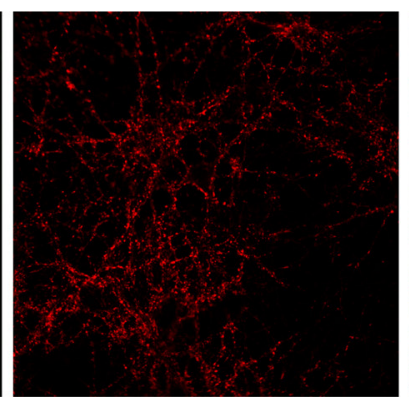

Merge
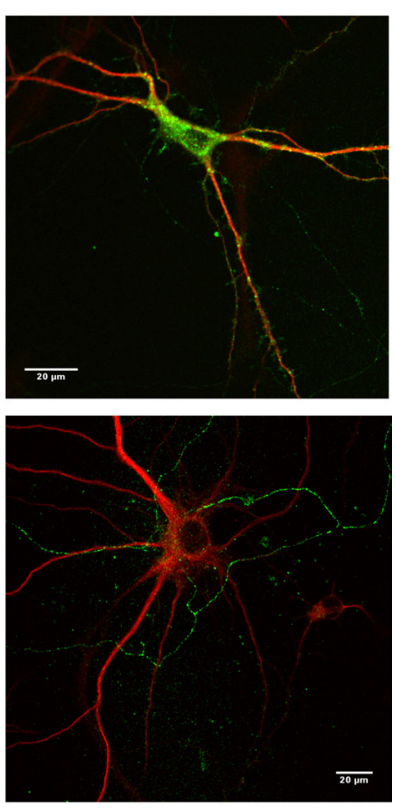

Merge

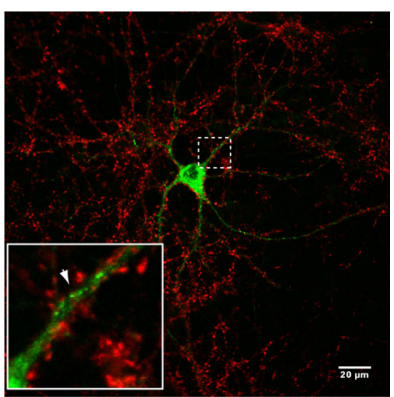

Figure 3.16: Rat hippocampal cultures (E18, 14 DIV) transfected with (hSyn)rEAG1GFP (green). Co-staining with MAP2 (red, upper rows) reveals that overexpressed rEAG1 is present in the somato-dendritic compartment (upper row) but also in axons (middle row). Co-staining with p38 (lower row) shows little colocalization between both proteins. 


\section{Expression of Venus-CaM and Venus-apoCaM in dissociated embryonic rat hippocampal cultures}

Endogenous CaM is expressed ubiquitously and abundantly in neuronal tissue, predominantly in neurons, but is also present in glia [11,14,91]. In neuronal cells CaM is expressed foremostly in the soma and dendrites [11]. Axons and pre-synapses have CaM in smaller amounts [11].

Venus-CaM and Venus-apoCaM constructs under the control of the human Synapsin promoter were transfected into E18 embryonic hippocampus cultures which were fixed and imaged at 10-12 DIV. Both fusion proteins were expressed cytosolically throughout the cells. Fluorescently-labeled CaM was present only in small amounts in the nucleus, whereas the concentration of apoCaM was similar in both nucleus and cytoplasm.

There were morphological differences between cells expressing Venus-CaM and those expressing Venus-apoCaM. In general, cells expressing Venus-CaM had more outgrowths and more neurite branching than both apoCaM-expressing and nontransfected cells.

CaM has been described to translocate from the cytoplasm of neurons to the nucleus upon sustained depolarization (90 $\mathrm{mM} \mathrm{K}^{+}$for $180 \mathrm{~s}$.) [16]. This was verified for the cultures used in this work (Fig. 3.18).

Upon $\mathrm{K}^{+}$stimulation neither Venus-CaM- nor Venus-apoCaM- transfected cells displayed differences in the nuclear CaM concentration (Figs. 3.17). However, cells transfected with Venus-CaM did have an increase in the number and size of spiny outgrowths. Although the translocation of CaM to the nucleus has been shown to occur upon $\mathrm{Ca}^{2+}$ binding $[16,44]$, Venus-apoCaM was present in higher amounts the nucleus than its $\mathrm{Ca}^{2+}$-binding counterpart.

\subsubsection{FRET measurements in neurons co-transfected with rEAG1-Cerulean and Venus-CaM}

Dissociated hippocampal cultures of E18 rats were co-transfected with (hSyn)VenusCaM and (hSyn)rEAG1-Cerulean. Similarly to HEK293 cells, co-transfected neurons tend to display robust Venus-CaM fluorescence and only a faint rEAG1Cerulean signal. When cDNA transfection ratios of 3:1 (rEAG1/CaM, m/m) were 


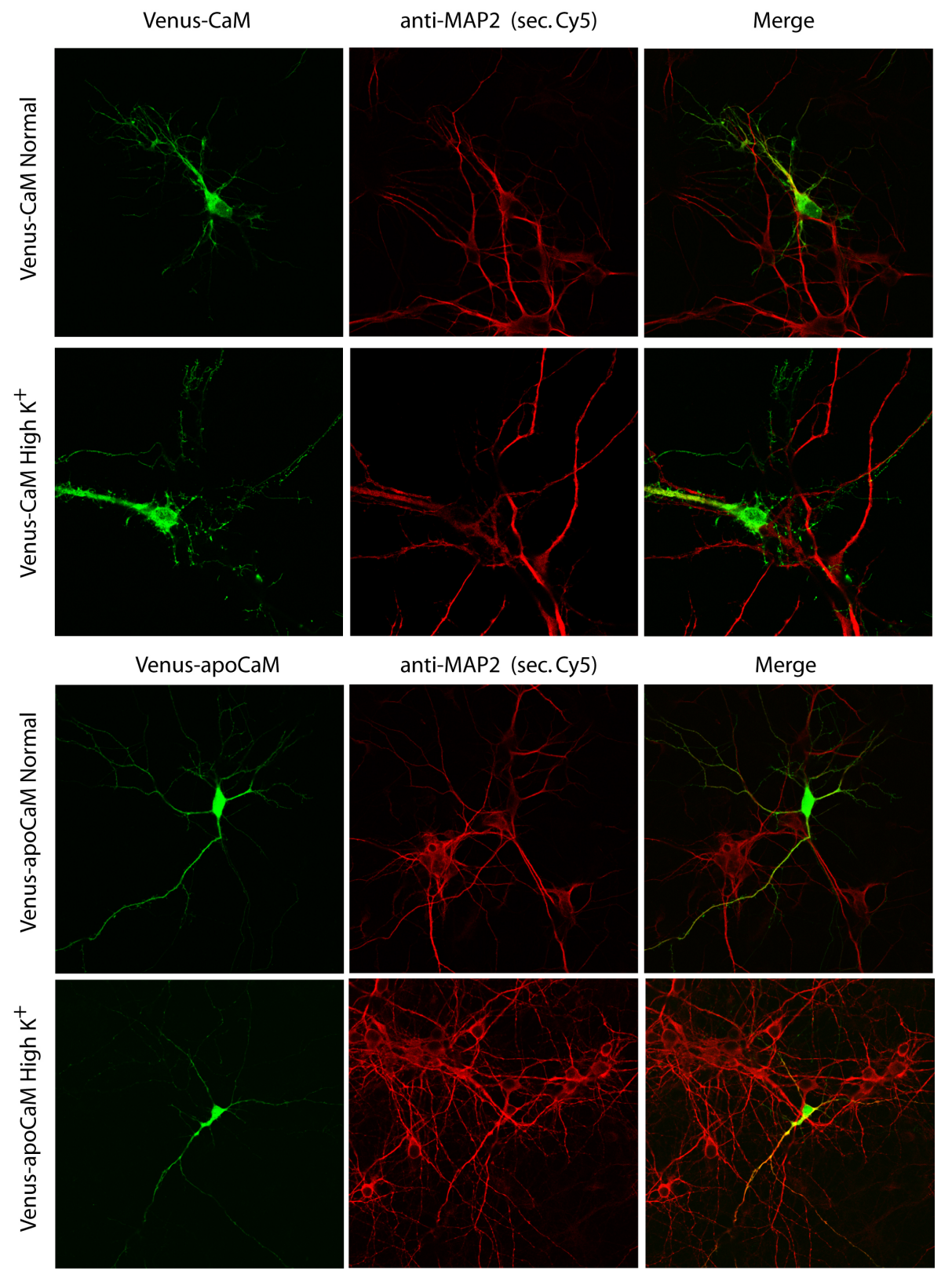

Figure 3.17: Overexpression of Venus-fused CaM and apoCaM (green) in rat hippocampal cultures (E18, 10-12 DIV). Co-staining with anti-MAP2 antibody (red). Both fluorescent-fusion proteins are expressed throughout the cytosol of neurons and only apoCaM is present in large amount in the nucleus. Neurons transfected with Venus-CaM show increased neuritogenesis and branching of processes. No translocation of CaM constructs occurred upon stimulation with $90 \mathrm{mM} \mathrm{K}^{+}$for $180 \mathrm{~s}$, however neurons transfected with Venus-CaM displayed increased fluorescence in filopodia/spine-like structures. 
anti-CaM (sec.Cy5)

Normal K+

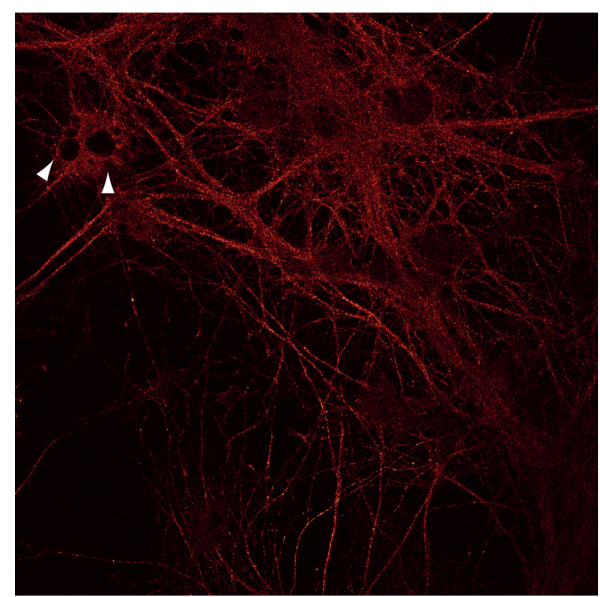

$\mathrm{High} \mathrm{K}^{+}(90 \mathrm{mM}, 180 \mathrm{~s})$
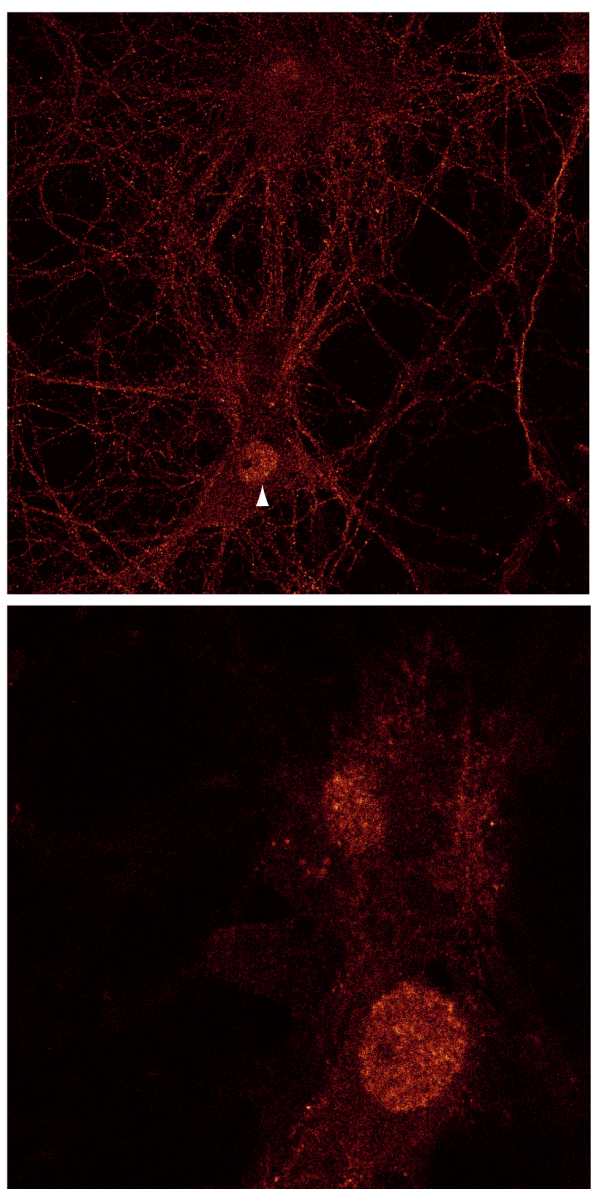

Figure 3.18: Translocation of endogenous CaM in rat hippocampal cultures (E18, 12 DIV) stained with anti-CaM antibodies. CaM translocates to the nucleus of neurons following stimulation with $90 \mathrm{mM} \mathrm{K}^{+}$for $180 \mathrm{~s}$ (see arrowheads). 
used only a small proportion of transfected cells had a detectable Cerulean signal, which was primarily located to vesicles in the soma. Transfection ratios of 5:1 or 10:1 (rEAG1/CaM, m/m) resulted in detectable levels of rEAG1-Cerulean in neurites.

FRET was measured by acceptor photobleaching in co-transfected, fixed neurons stimulated with $90 \mathrm{mM} \mathrm{K}^{+}$for $180 \mathrm{~s}$ or $1 \mu \mathrm{M}$ ionomycin in Ringer $\left(1.8 \mathrm{mM} \mathrm{Ca}^{2+}\right)$. In either case no interaction between rEAG1-Cerulean and Venus-CaM could be recorded, both in neurites and in the soma of transfected neurons, at different transfection ratios (Fig.3.19).

\section{The ratio of fluorescently-labeled to endogenous CaM is lower in trans- fected neurons than in HEK293 cells}

CaM is expressed endogenously in high amounts in neural tissue. In order to verify if the absence of measurable FRET in neurons was due to competition from nonfluorescent endogenous CaM, the ratio of endogenous to transfected CaM was determined in neuronal cultures co-transfected with rEAG1-Cerulean and Venus-CaM and also in HEK293 cells co-transfected with the corresponding CMV promoter constructs (Fig. 3.20).

Co-transfected cultures were stained with anti-CaM and antibodies and the average intensities of this staining were compared for several regions of interest (for neurons: both in the soma and neurites) in transfected and untransfected cells. Differences between average intensities of the CaM staining were normalized to the average intensity of CaM in non-transfected cells, in order to determine the ratio of VenusCaM/endogenous CaM. This was found to be 1.4 ( $\pm 0.2 \mathrm{SEM})$ in transfected HEK cells, but merely $0.2( \pm 0.1 \mathrm{SEM})$ in neurons, meaning that the majority of CaM present in transfected neurons is endogenous and unlabeled. Binding competition from endogenous CaM is therefore likely to account for the fact that no FRET is measurable between rEAG1-Cerulean and Venus-CaM in neurons. 

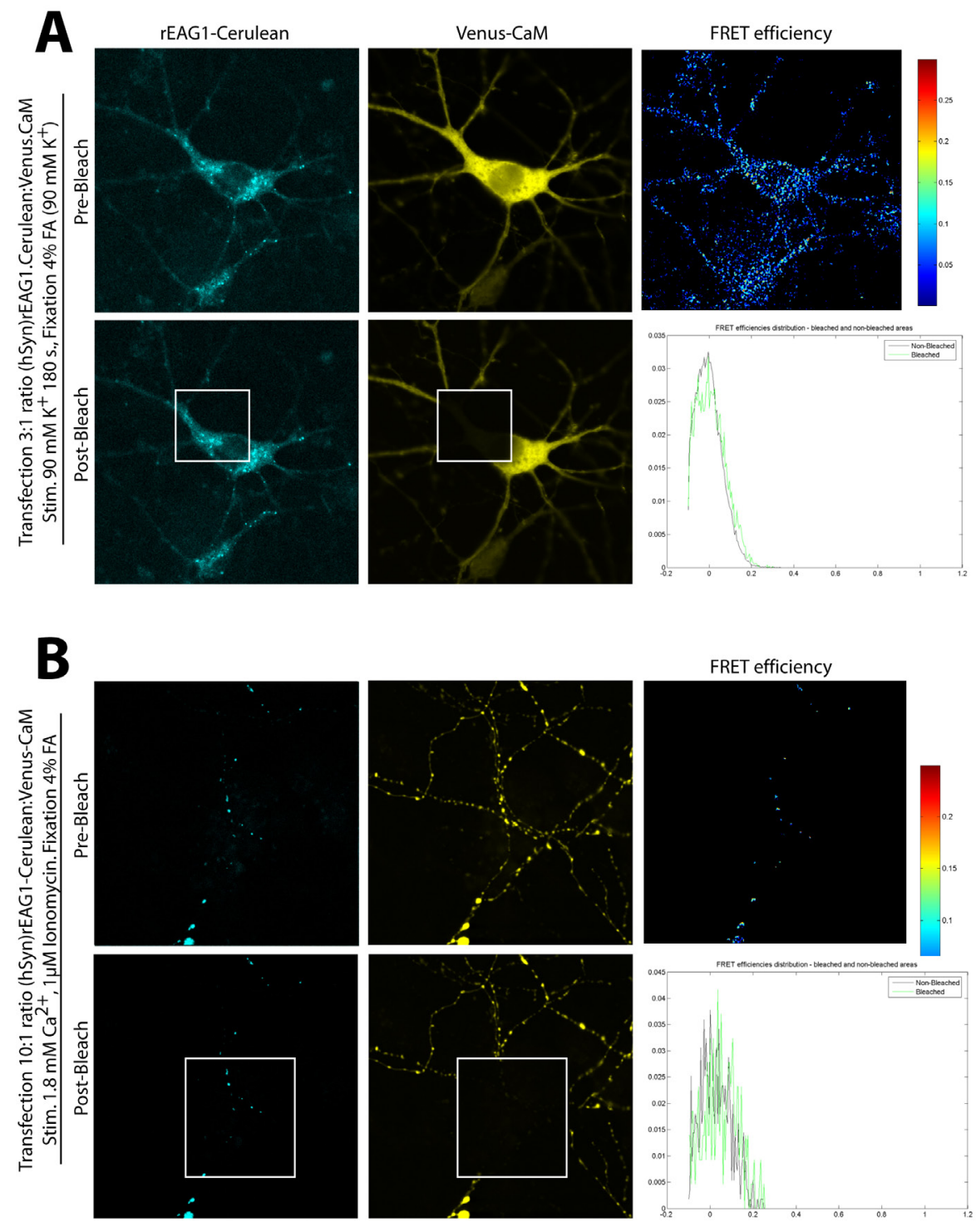

Figure 3.19: Acceptor photobleaching FRET measurements between rEAG1-Cerulean and Venus-CaM in co-transfected rat hippocampal cultures (E18, 12 DIV). No FRET was measured both in the soma and processes of co-transfected neurons, after stimulation with $90 \mathrm{mM} \mathrm{K}^{+}$or $1 \mu \mathrm{M}$ ionomycin in the presence of $\mathrm{Ca}^{2+}$. Neurons transfected with DNA ratios of 3:1 (EAG1:CaM, m/m) had little or no detectable signal in neurites, allowing measurements only in the soma (A). DNA ratios of 10:1 resulted in EAG1 being detected in neuronal processes (B). 

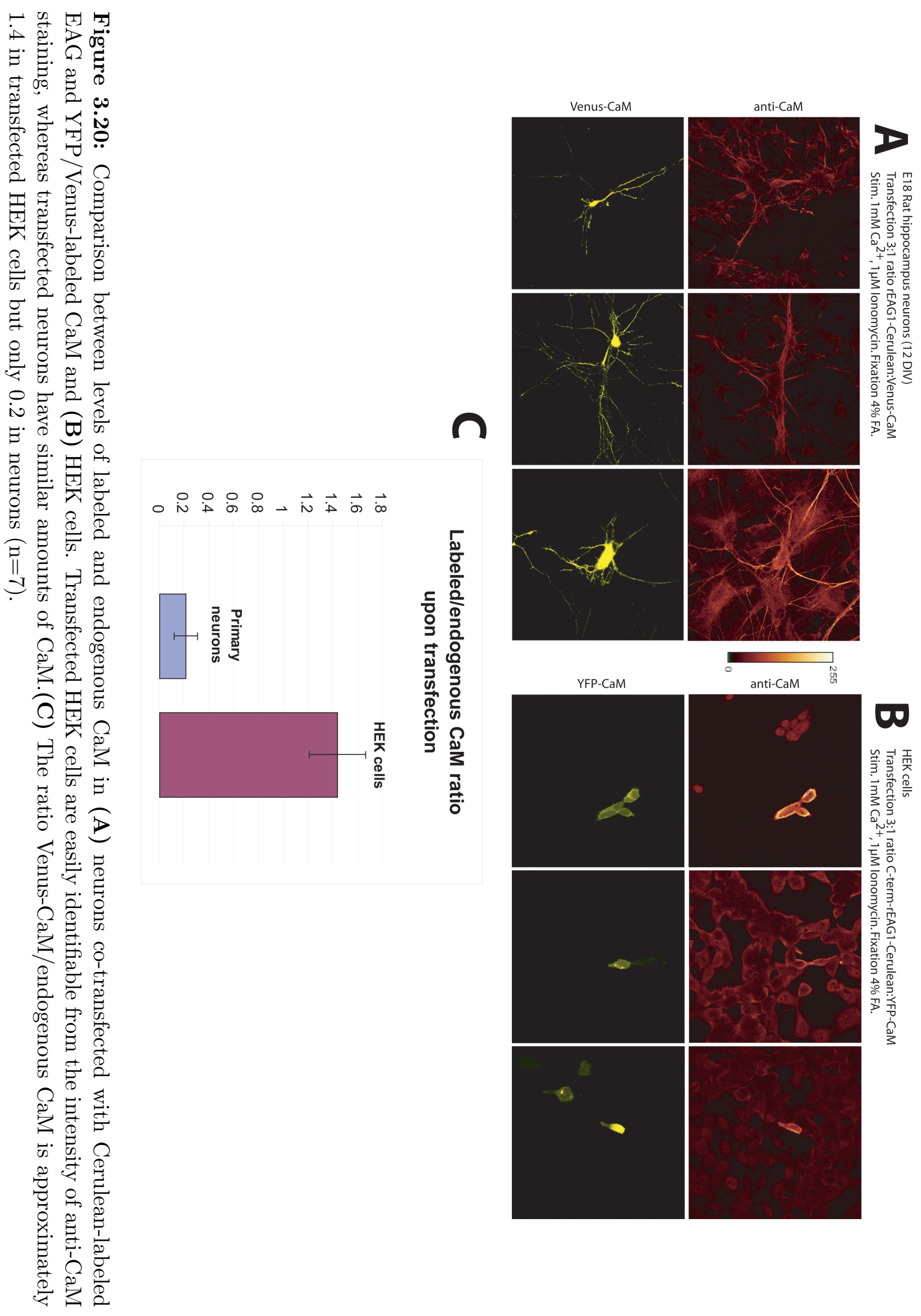


\section{Discussion}

\subsection{Photoconversion of Yellow Fluorescent Pro- teins}

A part of the present work describes the permanent photochemical conversion of YFP to a fluorescent species (XFP) with an emission maximum of $470 \mathrm{~nm}$, upon 'photobleaching' with $514 \mathrm{~nm}$ illumination. The emission maximum of the XFP photoconversion product closely matches that of cyan fluorescent proteins, making it difficult to separate the emission of both species and thereby interfering with acceptor photobleaching FRET measurements [4]. Acceptor photobleaching is a relatively simple and cost-effective method of measuring FRET in a cellular environment, especially in intermolecular assays, because it is relatively free from constraints of different donor/acceptor expression levels which frequently affect other intensitybased FRET methods. Acceptor photobleaching experiments using the CFP/YFP FRET pair (or their derivatives) [46] are one the most widely used FRET experiment configurations, the artifacts arising from the photoconversion of YFP are therefore of great relevance.

Yellow fluorescent proteins are long known to undergo intensity fluctuations (blinking [47]) and reversible photobleaching [82] - fluorophores photobleached with 514 $\mathrm{nm}$ light spontaneously recover their fluorescence over hours or over seconds when illuminated in the 330-390 nm range. However, a complete spectroscopic study of the photochemistry of YFP was only recently reported [42]. McAnaney et al. describe the irreversible formation of a weakly fluorescent product upon prolonged illumination of YFP. This product (termed XFP) was found to have absorption and emission maxima at 390 and $460 \mathrm{~nm}$, respectively. This is compatible with the findings of our work, in particular with the fact that the fluorescence emission of the photoconversion product was strongest upon $405 \mathrm{~nm}$ excitation (i.e., close to the $390 \mathrm{~nm}$ 
absorption peak of XFP) and much fainter under $458 \mathrm{~nm}$ illumination. Velentin et al. [85] reported similar data, based on photobleaching experiments performed with YFP and its derivatives Citrine and Venus.

The photoconversion of YFP and its derivatives should be considered when performing photobleaching experiments. The photoconversion product XFP is weakly fluorescent but can interfere with FRET measurements under specific circumstances, namely whenever a high concentration of YFP is present. The concentration of YFP will determine how much XFP is formed during photobleaching. Nevertheless, the contribution XFP fluorescence to measured FRET efficiencies will depend on the concentration of CFP, since relative increases in the fluorescence of the donor are measured. Experiments where both proteins of interest are homogeneously expressed in the cytosol are not likely to display effects of photoconversion (and this is probably why this process went so long unnoticed). However, if the donor is present in small structures and the acceptor is cytosolic, as when measuring the interaction of EAG with CaM, then there is a high probability that XFP fluorescence will affect the measured FRET efficiencies.

The proportion of XFP fluorescence on the donor image can be kept to a minimum by exciting CFP with a wavelength as far a possible from the $390 \mathrm{~nm}$ absorption peak of XFP. Lately, $405 \mathrm{~nm}$ lasers have been promoted as a good alternative to the $458 \mathrm{~nm}$ line of Ar lasers, which is relatively faint. However, our results show that $458 \mathrm{~nm}$ can drastically reduce the excitation of the YFP photoconversion product (Fig. 3.3), and are therefore more suitable for use in acceptor photobleaching FRET experiments. Recently new solid-state $430 \mathrm{~nm}$ lasers have become available that would probably be a good alternative to $458 \mathrm{~nm}$. Despite being closer to the excitation maximum of XFP than $458 \mathrm{~nm}$, these lasers are able to optimally excite CFP which has an absorption maximum precisely at $430 \mathrm{~nm}$.

The CFP/YFP FRET pair was already known to have problems caused the low brightness of CFP (see §1.2.1). The photoconversion of YFP now presents a further disadvantage for the use of these chromophores. A large number of new fluorescent proteins has recently become available which are likely to finally render the CFP/YFP pair obsolete. However, the photoconversion of yellow FPs could prove to be useful in some cases, such as the possibility of using YFP as a highlightable protein for tracking experiments. Though far from being a good alternative to purpose-designed photoconvertible proteins such as Kaede [83], YFP-fusion proteins are used in a many transgenic mice where the cost of cloning a new high- 
lightable protein would probably outweigh the benefits. In cases where the intensity of the photoconverted protein permits its tracking this possibility should not be discarded.

In conclusion, FRET experiments using the CFP/YFP pair and their derivatives should include a non-interacting control which is in all similar to the YFP-fused protein but where no FRET is expected. In this work the CaM mutant with impaired $\mathrm{Ca}^{2+}$ binding apoCaM was used. In experiments where photoconversion poses a problem stringent thresholding of areas with low donor expression is recommended. The different absorption maxima of XFP and CFP could potentially be used to spectrally separate the two chromophores.

\subsection{Mechanism of $\mathrm{Ca}^{2+} / \mathrm{CaM}$ inhibition of EAG channels and potential functional implica- tions}

Several studies $[8,38]$ have suggested a role for EAG channels in the regulation of neuronal excitability, possibly by contributing to the maintenance of the resting membrane potential. EAG channels mediate inward-rectifying currents with activation close to the membrane resting potential which would be compatible with such a physiological function. The dependence of the EAG currents on the holding voltage (Cole-Moore effect) [38] and the regulation of their gating by extracellular $\mathrm{Mg}^{2+}$ [78] give further support to this hypothesis.

Rat and human EAG1 were found to be inhibited by $\mathrm{Ca}^{2+}$ with a half-maximal inhibition occurring at a $\mathrm{Ca}^{2+}$ concentration of $\sim 100 \mathrm{nM}$ [74]. Schönherr et al. [70] reported that this inhibition was mediated by the binding of $\mathrm{Ca}^{2+} / \mathrm{CaM}$ to the $\mathrm{C}$ terminus of the channel. Calcium levels are a good correlate of neuronal activity and membrane depolarization. $\mathrm{Ca}^{2+}$-activated potassium channels (such as BK, IK and SK) establish a negative feedback loop whereby depolarization-induced high $\mathrm{Ca}^{2+}$ levels activate re-polarizing $\mathrm{K}^{+}$currents [75]. Interestingly, EAG channels close in response to increases in $\mathrm{Ca}^{2+}$ just above the resting concentration, thus exerting a positive feedback on neuronal activity.

The presence of these regulatory mechanisms must exert a tight control on the activity of the channel. The Cole-Moore effect and the $\mathrm{Mg}^{2+}$ regulation hinder 
current activation after hyperpolarization whereas $\mathrm{Ca}^{2+}$ could inhibit the channel during and following neural activity, during which $\mathrm{Ca}^{2+}$ levels are usually high. Although these mechanisms of regulation have been studied, the actual physiological function of EAG is not known. The inhibition of EAG1 currents by CaM at closeto-resting $\mathrm{Ca}^{2+}$ concentrations is perhaps the factor that will most contribute to the physiological function of the channel, as it is expected to severely limit its open time.

The present work aimed at further understanding the $\mathrm{Ca}^{2+} / \mathrm{CaM}$-mediated regulation of EAG channels in order to obtain a an insight in its possible physiological significance in neurons.

\subsubsection{FRET assay for CaM binding to EAG}

\section{Expression of fluorescent fusion constructs of EAG}

Membrane proteins, such as ion channels, usually display a characteristic membrane contour staining when expressed as fluorescent fusion constructs or detected with fluorescent antibodies. In the case of EAG1 such a membrane contour is seldom visible. The channel is present predominantly in the cytosol in the form of small vesicles. Both hEAG1 and especially rEAG2 are prone to form aggregates in the ER/Golgi and perinuclear regions when expressed in mammalian cell lines. However, this is the case both for the wild-type channels as for their Cerulean-fusion counterparts. Furthermore, the electrophysiological properties of the overexpressed, fluorescently-labeled channels appear to be conserved.

Members of the EAG channel family have been shown to have an ER retention signal of the RXR type [96]. This was first reported for hERG [33], where mutation of the RGR sequence at aa. 1005-1007 results in larger current densities being measured, reflecting a larger number of channels in the cellular membrane, which was confirmed by a biotinylation assay for membrane expression. It has been proposed that this ER retention signal prevents the Kir 6.1/2 and $\mathrm{GABA}_{B}$ receptor subunits from leaving the ER before they are assembled in a functional tetrameric form $[40,96]$. Conformational changes that occur upon tetramerization of the subunits would mask these signaling sequences, thus permitting the trafficking of the channel to the membrane. In hEAG1 the RKR sequence at aa. 675-677 was mutated (to LKL) and shown to increase current densities when expressed in Xenopus 
oocytes and HEK293 cells ( [26], L. Pardo, personal communication). However, the expression pattern of the channel did not display significant changes in CFP-fusion constructs containing the same mutations, an indication that the increase in channel trafficking is not sufficient for visible changes in the fluorescence pattern to occur. The data shown in this work was obtained with EAG constructs containing an intact RKR signal.

One study [24] found that rEAG1 interacts with KCR1, a membrane protein that facilitates the functional expression of rEAG1 channel. This raises the prospect that regulatory subunits may play a role in the trafficking and membrane expression of EAG channels.

\section{Localization of EAG constructs in hippocampal neurons}

When expressed in dissociated hippocampal cultures, rEAG1-Cerulean displays a fluorescence pattern similar to that of HEK293 cells - no membrane countour staining, most of the fluorescent signal is present in small vesicles which concentrate mainly in the soma and perinuclear region (Fig. 3.16). Channels expressed in neurons have a smaller propensity to form aggregates in the ER/Golgi than in HEK cells, however, this could be an effect of low expression under the hSyn promoter and not necessarily cell-type related. Over-expressed rEAG1-Cerulean is present throughout the transfected neurons, not only in the somatodendritic compartment but also in axons. Whereas no endogenous rEAG1 could be detected with the 7194 antibody in hippocampal cultures, EAG2 appeared in both dendrites and MAP2 negative processes implying both axonal and dendritic localization.

There is presently a single published study on the subcellular localization of endogenous EAG channels. Jeng et al. [25] found that EAG was present only in the somata and dendrites of dissociated rat hippocampal neurons. In the same study the authors localized EAG to the post-synapse. This result was based on the fact that the stainings of EAG channels were present in MAP2 positive processes but did not colocalize with the axonal marker Tau. Moreover, co-localization of both channels with the synaptic marker p38, and of EAG1 with the glutamatergic post-synaptic marker Densin and EAG2 with the GABA-ergic pre-synaptic marker GAD indicate an excitatory synapse localization for EAG1 and inhibitory for EAG2. Nevertheless, it is questionable whether optical microscopy has the necessary resolution for localizing ion channels to either pro- or post-synapse. The distance between both synaptic terminals is about $20 \mathrm{~nm}$ [68], i.e., over ten times smaller than the res- 
olution of a confocal microscope. The authors argue that, should the localization be pre-synaptic, transport vesicles would be visible in the axons. However, electron microscopy would be a more direct assay for pre- or post-synaptic localization. A yet unpublished electron microscopy study done in sections of adult rats has found EAG1 to be present in the pre-synaptic terminals (M. E. Rubio, L. Pardo, personal communication). Furthermore, antibody stainings of adult rat brain sections revealed the presence of EAG1 in the Purkinje cell layer of the cerebellum but not in the granule or molecular layers (S. Martin, F. Queiroz, personal communication). Insitu hybridization studies have shown that mRNA coding for EAG1 is only present in the granule layer and to a lesser extent in the deep nuclei of the cerebellum $[39,66]$. It is therefore likely that the EAG1 found in the Purkinje layer is actually present in neurons establishing synapses onto Purkinje cells (in their proximal processes) and consequently that it is localized in the pre-synapse.

This conflicting data shows that the intracellular localization of EAG is still unclear. A synaptic localization seems consensual, although different studies suggest either pre-synaptic or post-synaptic localizations. This question is, however, essential for formulating any hypothesis on the function of EAG channels. The endogenous distribution of EAG would also be needed in order to verify the distribution of over-expressed rEAG1-Cerulean in hippocampal neurons.

\section{CaM expression and translocation}

Upon expression in HEK cells, fluorescent fusion-constructs of CaM and apoCaM were present in the cytoplasm and nucleus, their concentration in the nucleus being usually higher than in the cytoplasm (Fig. 3.5). The presence of CaM in the nucleus is believed to be related to its function as activator of transcription factors $[16,44]$. CaM is transported to the nucleus by passive diffusion and by facilitated mechanisms which can be both $\mathrm{Ca}^{2+}$-dependent and -independent [79]. Active transport is also seems to play a role in the nuclear transport of YFP-CaM and YFP-apoCaM, given that the nuclear concentration of both proteins is frequently higher than in that of the cytoplasm.

\section{Expression of CaM constructs in neurons}

In rat E18 hippocampal cultures (10-12 DIV) both CaM and apoCaM fusion constructs displayed a homogeneous cytosolic localization in the somatodendritic com- 
partment and axons (Fig. 3.17). Endogenous CaM is known to be expressed in all neural tissue at all ages [91], although its intracellular localization varies during development [11]. It is present at highest concentrations in the soma and dendrites of neurons.

CaM is known to translocate to the nucleus of neurons in response to synaptic inputs. Translocation to the nucleus occurs upon $\mathrm{Ca}^{2+}$ entry through post-synaptic L-type channels and NMDA receptors [16]. This effect can be induced in neuronal cultures by applying a depolarization pulse with $90 \mathrm{mM} \mathrm{K}^{+}$for $180 \mathrm{~s}$ [16] and could be reproduced under our culture conditions (E18 rat hippocampus, 10-12 days in vitro).

In transfected neurons, the concentration of fluorescent CaM is lower in the nucleus than in the surrounding cytoplasm. Moreover, no significant translocation of CaM is seen upon depolarization (Fig. 3.17). Paradoxically, apoCaM was present at the same concentration in both nucleus and cytoplasm. Again, no translocation of apoCaM is seen upon stimulation. The fact that apoCaM is present in equal amounts in nucleus and cytoplasm could be explained by passive diffusion or transport by $\mathrm{Ca}^{2+}$-independent mechanisms. The concentration of free apoCaM is expected to be far larger than that of CaM. This is because the latter tends to have more binding targets than the former. However, it is surprising that Venus-CaM did not translocate in a larger scale to the nucleus after a $180 \mathrm{~s}$ depolarization pulse, as reported by Mermelstein et al. [44].

Another feature of CaM-transfected neurons was their strikingly increased neuritogenesis and branching (Fig. 3.17). CaM is known to promote neuritogenesis through a molecular pathway involving CaM-dependent kinases (CaMK) I and II [23,90]. The availability of CaM is often a rate-limiting step in many reactions it catalyses $[36,57,63]$, which raises the possibility that CaM overexpression leads to morphological changes in neurons.

Neurons transfected with Venus-CaM display an apparent increase in the number of spines and filopodia upon stimulation. Dendritic spines are actin-rich and highly motile structures $[19,22,30]$ that contain the majority of post-synaptic densities in hippocampal neurons. It is known that CaM has a role in the changes of size and morphology of dendritic spines which take place after synaptic stimuli. Several studies have shown that these morphological changes are triggered by CaMKII $[56,64,65]$. Jourdain et al. [27] have shown that the rate of spine formation in hippocampal neurons was increased upon injection of CaM and that this effect was not present when 
CaM was co-injected with a specific inhibitor of CaMKII. However, although changes in spine shape can occur within seconds, spine volume changes and spine creation are processes which usually take a few minutes and up to one hour [56]. The increase in the number and size of fluorescent spines in neurons expressing Venus-CaM is therefore likely to be linked to the relocation of CaM/CaMKII complexes to dendritic spines [72]; i.e., the major change is in the concentration of Venus-CaM in spines even though small changes in volume and shape of the spine may occur.

\section{Co-expression of EAG and CaM/apoCaM fusion constructs}

One of the first steps in the design of a FRET experiment is the assignment of donor and acceptor fluorophores to the proteins being studied. When measuring FRET by acceptor photo-bleaching [4] only the intensity changes of the donor fluorophore are used for computing FRET efficiencies (see Eq. 1.4). Unbound donor molecules can hence reduce the apparent FRET efficiency. The stoichiometry of the interaction will therefore affect FRET efficiencies - more acceptor molecules per donor results in higher FRET efficiencies, as the probability of energy transfer is higher. Schönherr et al. [70] proposed that one CaM molecule is sufficient to block hEAG1, assuming that only one or possibly two CaM molecules would bind to the channel, a highly favorable 1:4 or 1:2 donor:acceptor ratio would be achieved labelling CaM with Cerulean and hEAG1 with YFP. However CaM is a cytosolic protein expressed in higher amounts and with a more disperse cytosolic pattern than hEAG1, changes in Cerulean fluorescence due to FRET are therefore bound to be diluted in the total CaM signal. Labeling hEAG1 with Cerulean permitted the measurement of FRET with YFP-CaM as acceptor.

For FRET experiments with fluorescently labelled EAG and CaM donor and acceptor constructs were co-transfected in different ratios. An excess of acceptor molecules is desireable for an increased probability of energy tranfer. This is usually achieved by transfecting more acceptor cDNA than donor. This approach was tried for different EAG:CaM transfection ratios but instead a mass excess of donor cDNA was found to be beneficial for FRET measurements. In this study the nature of the proteins of interest is significantly different - EAG is a 962 aa. long membrane protein that undergoes post-translational assembly and glycosilation [52] and CaM is a much shorter (148 aa.) cytosolic protein. Therefore production of EAG in cells is substantially slower than CaM and, in addition, EAG was labeled with a much fainter fluorophore. This resulted in low and noisy donor signals. The best donor:acceptor 
$(\mathrm{m} / \mathrm{m})$ transfection ratios were found to be 3:1 for HEK cells and 10:1 for neuronal cultures.

\section{Ratio of endogenous to overexpressed CaM in neurons}

FRET experiments performed in cells that endogenously express one of the interacting proteins face the additional problem of competition from unlabeled binding partners. This was found to occur in neurons co-transfected with Cerulean-fused rEAG1 and Venus-labeled CaM. CaM is one of the most abundant proteins in neurons, the ratio of fluorescently-labeled to endogenous CaM is only 0.2 in transfected hippocampal neurons whereas in HEK293 cells it is 1.4 (Fig. 3.20). Endogenous $\mathrm{CaM}$ is therefore more abundant and competes for binding with EAG1, thereby reducing the amount of molecules available for FRET. Since neurons are sensitive to protein overexpression, increasing the total amount of transfected DNA is not a viable solution, while increasing the proportion of transfected CaM to EAG1 is likely to result in the EAG Cerulean signal no longer being detected. A further disadvantage of overexpression of CaM is that small changes in CaM concentration will lead to large changes in the $\mathrm{Ca}^{2+}$-buffering of neurons, possibly leading to unphysiological situations. Using a fluorescently-labeled CaM antibody as a FRET acceptor would permit the measurement of FRET between endogenous CaM and fluorescent EAG, thus circumventing the problems of overexpression.

In order to measure FRET between EAG and genetically-labeled CaM in neurons the endogenous levels of EAG should be lowered or a CaM YFP knock-in mouse used. At present no fluorescent CaM transgenics have been reported. CaM is encoded in mammals by three genes (CaM1, CaM2, CaM3) which are concurrently expressed in most areas of the brain [58]. This poses a considerable challenge to the suppression of endogenous CaM by RNA interference, which could be aimed at the $5^{\prime}$ untranslated region of CaM mRNA so that the expression of the YFP-labeled construct is not affected.

Alternatively, a concatenated construct joining Cerulean-labeled rEAG1 and VenusCaM would enable the maintenance of a constant high local concentration of labeled CaM (see Mori et al. [50] for a similar approach). Such a construct would be good at sensing changes in $\mathrm{Ca}^{2+}$ concentration but less good for changes in the availability of CaM. 


\subsubsection{N-terminal CaM binding domain of EAG1 chan- nels}

hEAG1 channels were previously known to be inhibited by $\mathrm{Ca}^{2+} / \mathrm{CaM}$. Mutations in the CaM binding site at aa. 707-726 were found to prevent the binding of CaM to the C-terminus domain and consequently to prevent the inhibition of hEAG1 by CaM [70]. The FRET assay described in the present study found that the interaction of the C-term-rEAG1 with CaM could be fully inhibited by $\mathrm{Ca}^{2+}$ depletion whereas the same treatment merely reduced the interaction of CaM with the full-length hEAG1 (Fig. 3.5).

The differences in $\mathrm{Ca}^{2+}$-dependent binding of hEAG1 and the cytosolic C-terminus domain of rEAG1 could be due to a steric loss of function of the truncated protein (i.e. due to the destruction of intramolecular interactions) or due to the presence of further CaM-binding domains. It is less likely that any differences observed are due to distinctions in the aminoacid sequence of the two species. Rat and human EAG1 share $98 \%$ of their sequence and the same FRET assay done with rEAG1 revaled the same $\mathrm{Ca}^{2+}$ response (data not shown). However, as mentioned above, the rat variant of EAG1 was found to be largerly retained in the ER and Golgi apparatus.

The C-terminal CaMbd mutations described by Schönherr et al. [70] were found to abolish the binding of CaM to the C-terminus of EAG1 but not to the fulllength channel (Fig. 3.6). The FRET efficiencies measured for the mutated fulllength channel were approximately the same under high and low $\mathrm{Ca}^{2+}$ conditions, which is strongly indicative of the presence of a second binding domain with higher affinity for $\mathrm{Ca}^{2+} / \mathrm{CaM}$, at a site outside of the C-terminal tail. No interaction was found between apoCaM and EAG1, showing that the channel does not bind $\mathrm{Ca}^{2+}$-free CaM. Furthermore it is unlikely that the steric effects could account for the abolition of the CaM interaction in the truncated C-terminus containing the CaMbd mutations: Schönherr et al. have found that the $K_{D}$ of the binding of CaM to the segment $673-867$ of hEAG1 was compatible with the half-maximal inhibition of $\sim 100 \mathrm{nM}$ found in electrophysiology studies, assuming the binding of one molecule of CaM to each channel tetramer is sufficient for current inhibition [70].

A CaM binding domain was found to be present in the N-terminus of EAG1. An overlay blot assay found direct binding of CaM to the segment aa. 147-209 of EAG1 (Fig. 3.8), while mutations F151S, A152S in this segment have localized the binding 
to a 1-8-14 CaM binding motif at aa. 151-164 (Fig. 3.9). This type of CaM binding motifs usually only interact with $\mathrm{Ca}^{2+}$-bound $\mathrm{CaM}$ and have high binding affinities [60]. That is compatible with the finding that CaM binding to EAG is detected even under low $\mathrm{Ca}^{2+}$ conditions. Channels containing both N- and C-terminal CaMbd mutations were found not to interact with CaM, as no FRET could be measured between both constructs, meaning that these two binding sites are essential for the binding of CaM to EAG1 channels (Fig. 3.10). Other parts of the channel may influence CaM binding to these primary sites, for example the aa. 668-679 sequence of the C-terminus which was found to have a structure favorable to CaM binding in the in silico screening (see 3.2.1).

A newly published study by Ziechner et al. [99] found evidence for the binding of CaM to the 1-8-14 motif in the N-terminus (aa. 151-165), thus confirming the existence of this second CaM binding domain. A peptide array scan also found the binding of CaM to the segments aa. 674-683 and 711-721, both in the C-terminus of the channel. These segments correspond to the putative binding domain found in silico and to the previously reported C-terminal CaMbd [70]. The same work was nevertheless inconclusive about the ability of the newly found C-terminal segment to bind CaM. Binding was found in the peptide scan and by confocal Fluorescence Correlation Spectroscopy (FCS) using a short ( 20 aa.) peptide. Moreover, channels with mutations in this putative binding region showed some reduction in current inhibition by $\mathrm{Ca}^{2+} / \mathrm{CaM}$. However, a FCS study using longer GST-fusion segments found no binding to CaM, the authors therefore speculate that this site is unable to bind CaM independently. This view is supported by our data which shows that no CaM binding occurs when the other two sites are disrupted by mutations (Fig. $3.10)$.

Ziechner et al. [99] found that both $\mathrm{N}$ - and C-terminal CaM binding sites were essential for the inhibition of hEAG1 currents by $\mathrm{Ca}^{2+} / \mathrm{CaM}$, as mutations in either site impaired $\mathrm{Ca}^{2+}$-driven current reductions. The relative CaM affinity of both sites was not entirely clear - the N-terminus (aa. 1-206) was found to have approximately the same binding affinity for $\mathrm{Ca}^{2+} / \mathrm{CaM}$ as the C-terminus (aa. 649-867) in FCS and Surface Plasmon Resonance (SPR) studies. However, when the FCS assay was repeated with short peptides the N-terminus, CaMbd was found to have a higher affinity than that of the C-terminus $\left(\mathrm{K}_{D}=45\right.$ and $223 \mathrm{nM}$, respectively). Our data is clearly in favor of the latter situation: the N-terminus binding domain was found to interact with $\mathrm{CaM}$ even in $\mathrm{Ca}^{2+}$-depleted cells, reflecting a higher affinity than that of the C-terminus. 
The present study therefore confirms and disambiguates the data of Ziechner et al., thereby highlighting the potential of FRET for the study of molecular interactions. Whereas FRET measurements were performed in a cellular environment with the membrane-inserted channels in their native state, biochemical and FCS assays used segments of channel subunits, the length of which was shown to affect the experimental outcome. Nevertheless, there is conclusive evidence from both studies for the presence of a further CaM binding domain in the N-terminus part of hEAG1.

\subsubsection{Molecular model of EAG1 inhibition by CaM}

The molecular model of the interaction of hEAG1 and CaM is currently facing a revision, following the discovery that CaM needs to bind to both $\mathrm{N}$ - and C-terminus for current inhibition to occur. The N-terminus of EAG channels is thought to play a role in channel gating, as mutations and deletions of this sequence cause changes in current activation and deactivation [77]. In fact Ziechner et al. [99] reported that mutations in the N-terminal CaMbd resulted in more positive activation voltages. Work by Terlau et al. and Morais-Cabral et al. [10,77] suggests that the N-terminus may interact with the S4-S5 intracellular linker of the EAG-family channels, which is itself thought to be involved in channel gating.

The mechanism of CaM inhibition of EAG appears to be substantially different to that of $\mathrm{Ca}^{2+}$-activated potassium channels, which have been shown to constitutively bind CaM independently of $\mathrm{Ca}^{2+}$ concentration (see $[67,75]$ for a review). However, two channel families with structural and functional similarities to EAG were also found to contain CaM binding domains, namely Cyclic Nucleotide Gated (CNG) $[81,92]$ and KCNQ channels [93,95].

Potassium channels of the KCNQ family have several similarities with EAG, both in terms of structure and electrophysiological properties. In fact, both channels have been proposed as mediators of the M-current, a low-voltage threshold potassium current which is inhibited by muscarinic acetylcholine and other receptors [73]. KCNQ channels have been shown to constitutively bind CaM through an IQ-like CaM binding motif [60], CaM bound to the channel in this manner was shown to mediate the inhibition of the channels by $\mathrm{Ca}^{2+}$. The mechanism of $\mathrm{Ca}^{2+} / \mathrm{CaM}$ regulation of these channels is therefore different from that of EAG channels, despite their structural and electrophysiological similarities.

CNG channels are the closest structural relatives of the EAG channel family. They 
share several structural features with EAG including a cyclic-nucleotide gating domain, that, although partially conserved from a sequence point of view, was found not to be functional in EAG1. In CNG channels, a CaM binding domain was shown to be present in the N-terminus of CNGB1 subunits and to mediate an intersubunit interaction with the C-terminus of CNGA subunits [92]. CaM in its $\mathrm{Ca}^{2+}$-associated form has been shown to bind to the CaMbd of CNGB1, thereby disrupting the intersubunit interaction and inhibiting the channel [81]. In order to verify if such an inhibition mechanism is present in EAG1 (Fig. 4.21) the binding of $\mathrm{N}$ - and C-terminus was tested on an overlay blot both in the presence and absence of $\mathrm{Ca}^{2+} / \mathrm{CaM}$ (data not shown). In both cases, no interaction between both intracellular domains could be recorded. This was is in accordance with the results of Ziechner et al. [99], who could not find an interaction between N- and C-terminal protein fragments of hEAG1 in precipitation assays. However, low affinity interactions can be difficult to detect using standard biochemical methods. Ion channels are highly dynamic structures that can have large conformational changes between open and closed states. These structural subtleties are irreproducible in many biochemical assays. Preliminary FRET data, obtained with fluorescent fusion constructs of both cytosolic termini indicates that an interaction could potentially occur. A FRET assay for conformational changes of olfactory CNG channels was reported by Zheng et al. [97] and allows the monitoring of the distance between intracellular domains of the channel with simultaneous electrophysiological recordings under different $\mathrm{Ca}^{2+}$ concentrations. The same approach could potentially be used to elucidate the molecular mechanism behind $\mathrm{Ca}^{2+} / \mathrm{CaM}$ ihibition of hEAG1.

The presence of two CaMbd in hEAG1 subunits also suggests that both N- and C-terminal binding domains could bind one single CaM molecule, thereby inducing a conformational change of the channel. This seems unlikely, as Ziechner et al. [99] found that both $\mathrm{N}$ - and C-terminal CaMbd both bind to the C-lobe of CaM, containing EF-hand domains 3 and 4, indicating that probably up to eight CaM molecules can bind to the channel. This is supported by the high FRET efficiencies measured, even in the presence of competition from endogenous CaM in HEK cells.

The N-terminus was found to have a very high affinity for $\mathrm{Ca}^{2+} / \mathrm{CaM}\left(\mathrm{K}_{D}=45\right.$ $\mathrm{nM} \mathrm{Ca}{ }^{2+}$ in one estimate [99]), raising the prospect that CaM could be a nearlypermanent constituent of hEAG1 channels. This is however dependent on the local concentrations of $\mathrm{Ca}^{2+}$ and availability of CaM to the channels, further highlighting the importance of the channel localization for modeling the function of EAG1. 

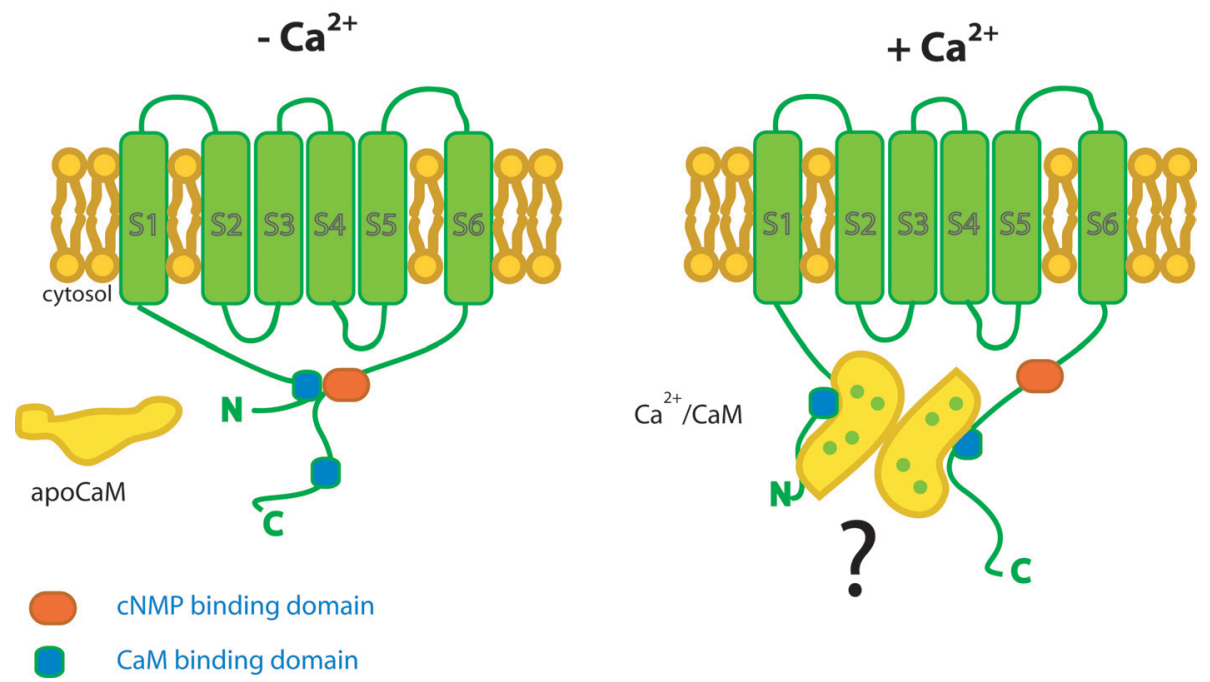

Figure 4.21: Schematic representation of a possible cooperative mechanism of N- and C-terminus for activity regulation of EAG, in which an interaction between both cytosolic termini is disrupted by the binding of $\mathrm{Ca}^{2+} / \mathrm{CaM}$. This hypothesis is in analogy to intersubunit interactions in CNG channels where the cytosolic N-terminus binds to the C-terminus in the region of the cyclic nucleotide binding domain.

\subsubsection{EAG localization, inhibition and function}

The interaction with $\mathrm{Ca}^{2+} / \mathrm{CaM}$ appears to be, a par with its voltage sensitivity, the dominant mechanism of EAG current regulation. The concentration of $\mathrm{Ca}^{2+}$ near the membrane can be extremely heterogeneous, in particular in the vicinity of $\mathrm{Ca}^{2+}$-channels $[5,48]$ and, therefore, small differences in localization can have a large impact on EAG currents.

The simplest functional mechanism of action of EAG channels would be one where an increase in $\mathrm{Ca}^{2+}$ inhibits EAG currents, thereby facilitating depolarization. EAG would then act as a $\mathrm{Ca}^{2+}$ sensor, repressing depolarizations which are not accompanied by an increase in $\mathrm{Ca}^{2+}$ concentration. The task of repolarizing the cell would then be left to other (e.g., delayed rectifier) channels. However, given that the $\mathrm{Ca}^{2+} / \mathrm{CaM}$ inhibition occurs so close to the resting $\mathrm{Ca}^{2+}$ levels, one would expect either limited channel activity or a specific localization away from $\mathrm{Ca}^{2+}$ channels.

$\mathrm{Ca}^{2+}$ regulates EAG through CaM, a molecule that, although abundant, has numerous binding partners, some of which are permanently bound with high affinity. A situation where competition for CaM underlies the regulation of EAG is therefore not to be excluded. It has been suggested that CaM can be locally depleted 
under certain circumstances [57]. Though speculative, a mechanism of local CaM depletion is supported by other examples of rapid CaM translocation, e.g., to the nucleus [16] or to spines [72]. Should activity/ $\mathrm{Ca}^{2+}$-induced competition for CaM be present, open EAG channels would be able to counter mounting neuronal activity establishing a negative feedback, an approach which is usually favored by nature. 


\section{Summary}

Several studies $[8,38]$ have suggested a role for EAG channels in the regulation of neuronal excitability, possibly by contributing to the maintenance of the resting membrane potential. EAG channels mediate inward-rectifying currents with activation close to the membrane resting potential which would be compatible with such a physiological function. Knowledge on the regulation of the activity of this kind of channels is therefore potentially important for the study of neuronal signaling.

EAG1 has been known to be inhibited by intracellular $\mathrm{Ca}^{2+}$ with a half maximal concentration of $100 \mathrm{nM}$ and this inhibition has been shown to be mediated by direct binding of calmodulin (CaM) to the C-terminal domain (aa. 707-726) of the channel [70]. The regulation of EAG activity was studied by visualizing its interaction with CaM, using an intermolecular FRET approach. FRET was measured between YFPlabeled CaM and several Cerulean-labeled EAG constructs. The fusion-proteins were expressed in HEK cells and the interaction was found to be $\mathrm{Ca}^{2+}$-dependent as the FRET efficiency decreases with increasing $\mathrm{Ca}^{2+}$ concentrations and is abolished when using a $\mathrm{Ca}^{2+}$-insensitive $\mathrm{CaM}$ mutant.

Mutations in the previously described CaM binding site at aa. 707-726 were found to prevent the binding of $\mathrm{CaM}$ to the $\mathrm{C}$-terminus domain and consequently to prevent the inhibition of hEAG1 by CaM [70]. The FRET assay described in the present study found that the interaction of the C-term-rEAG1 with CaM could be fully inhibited by $\mathrm{Ca}^{2+}$ depletion, whereas the same treatment merely reduced the interaction of CaM with the full-length hEAG1. This interaction was found to be accounted for by the presence of a further, high affinity CaM binding domain in the N-terminus of the channel at aa. 151-164.

In conclusion the present results show that two CaM binding sites are present in hEAG1 subunits - a high-afinity CaM binding domain of the form 1-8-14 in the $\mathrm{N}$-terminus and the previously described C-terminal binding domain at aa. 707- 
276. Mutations at these sites lead to the complete disruption of CaM binding to the hEAG1, these two binding domains are therefore responsible for CaM binding. These findings indicate that the regulation of hEAG1 by $\mathrm{Ca}^{2+} / \mathrm{CaM}$ is more complex than previously thought. 


\section{Bibliography}

[1] S. F. Altschul, W. Gish, W. Miller, E. W. Myers, and D. J. Lipman. Basic local alignment search tool. J Mol Biol, 215(3):403-410, Oct 1990.

[2] Y. S. Babu, J. S. Sack, T. J. Greenhough, C. E. Bugg, A. R. Means, and W. J. Cook. Three-dimensional structure of calmodulin. Nature, 315(6014):37-40, 1985 .

[3] G. A. Banker and W. M. Cowan. Rat hippocampal neurons in dispersed cell culture. Brain Res, 126(3):397-342, May 1977.

[4] P. I. Bastiaens, I. V. Majoul, P. J. Verveer, H. D. Soeling, and T. M. Jovin. Imaging the intracellular trafficking and state of the AB5 quaternary structure of cholera toxin. EMBO J, 15(16):4246-4253, Aug 1996.

[5] U. Becherer, T. Moser, W. Stuehmer, and M. Oheim. Calcium regulates exocytosis at the level of single vesicles. Nat Neurosci, 6(8):846-853, Aug 2003.

[6] N. Billinton and A. W. Knight. Seeing the wood through the trees: a review of techniques for distinguishing green fluorescent protein from endogenous autofluorescence. Anal Biochem, 291(2):175-197, Apr 2001.

[7] C. B. Brewer. Cytomegalovirus plasmid vectors for permanent lines of polarized epithelial cells. Methods Cell Biol, 43 Pt A:233-245, 1994.

[8] A. Brueggemann, L. A. Pardo, W. Stuehmer, and O. Pongs. Ether--go-go encodes a voltage-gated channel permeable to $\mathrm{K}+$ and $\mathrm{Ca} 2+$ and modulated by cAMP. Nature, 365(6445):445-448, Sep 1993.

[9] G. Bunt and F. S. Wouters. Visualization of molecular activities inside living cells with fluorescent labels. Int Rev Cytol, 237:205-277, 2004.

[10] J. H. M. Cabral, A. Lee, S. L. Cohen, B. T. Chait, M. Li, and R. Mackinnon. Crystal structure and functional analysis of the HERG potassium channel N terminus: a eukaryotic PAS domain. Cell, 95(5):649-655, Nov 1998.

[11] A. Caceres, P. Bender, L. Snavely, L. I. Rebhun, and O. Steward. Distribution and subcellular localization of calmodulin in adult and developing brain tissue. Neuroscience, 10(2):449-461, Oct 1983. 
[12] P. D. Camilli, P. E. Miller, F. Navone, W. E. Theurkauf, and R. B. Vallee. Distribution of microtubule-associated protein 2 in the nervous system of the rat studied by immunofluorescence. Neuroscience, 11(4):817-846, Apr 1984.

[13] R. E. Campbell, O. Tour, A. E. Palmer, P. A. Steinbach, G. S. Baird, D. A. Zacharias, and R. Y. Tsien. A monomeric red fluorescent protein. Proc Natl Acad Sci U S A, 99(12):7877-7882, Jun 2002.

[14] W. Y. Cheung. Cyclic 3',5'-nucleotide phosphodiesterase. Demonstration of an activator. Biochem Biophys Res Commun, 38(3):533-538, Feb 1970.

[15] K. S. Cole and J. W. Moore. Potassium ion current in the squid giant axon: dynamic characteristic. Biophys J, 1:1-14, Sep 1960.

[16] K. Deisseroth, E. K. Heist, and R. W. Tsien. Translocation of calmodulin to the nucleus supports CREB phosphorylation in hippocampal neurons. Nature, 392(6672):198-202, Mar 1998.

[17] S. S. Easter, L. S. Ross, and A. Frankfurter. Initial tract formation in the mouse brain. J Neurosci, 13(1):285-299, Jan 1993.

[18] B. Engeland, A. Neu, J. Ludwig, J. Roeper, and O. Pongs. Cloning and functional expression of rat ether--go-go-like $\mathrm{K}+$ channel genes. J Physiol, 513 ( Pt 3):647-654, Dec 1998.

[19] M. Fischer, S. Kaech, D. Knutti, and A. Matus. Rapid actin-based plasticity in dendritic spines. Neuron, 20(5):847-854, May 1998.

[20] T. Foerster. Zwischenmolekulare energiewanderung und fluoreszenz. Ann. Phys., 437:55-75, 1948.

[21] B. Ganetzky and C. F. Wu. Neurogenetic analysis of potassium currents in Drosophila: synergistic effects on neuromuscular transmission in double mutants. J Neurogenet, 1(1):17-28, Sep 1983.

[22] A. Gazzaley, S. Kay, and D. L. Benson. Dendritic spine plasticity in hippocampus. Neuroscience, 111(4):853-862, 2002.

[23] Y. Goshima, S. Ohsako, and T. Yamauchi. Overexpression of Ca2+/calmodulin-dependent protein kinase II in Neuro2a and NG108-15 neuroblastoma cell lines promotes neurite outgrowth and growth cone motility. $J$ Neurosci, 13(2):559-567, Feb 1993.

[24] N. Hoshi, H. Takahashi, M. Shahidullah, S. Yokoyama, and H. Higashida. KCR1, a membrane protein that facilitates functional expression of noninactivating $\mathrm{K}+$ currents associates with rat EAG voltage-dependent $\mathrm{K}+$ channels. J Biol Chem, 273(36):23080-23085, Sep 1998.

[25] C.-J. Jeng, C.-C. Chang, and C.-Y. Tang. Differential localization of rat Eag1 and Eag2 K+ channels in hippocampal neurons. Neuroreport, 16(3):229-233, Feb 2005. 
[26] M. Jenke, A. Snchez, F. Monje, W. Stuehmer, R. M. Weseloh, and L. A. Pardo. C-terminal domains implicated in the functional surface expression of potassium channels. EMBO J, 22(3):395-403, Feb 2003.

[27] P. Jourdain, K. Fukunaga, and D. Muller. Calcium/calmodulin-dependent protein kinase II contributes to activity-dependent filopodia growth and spine formation. J Neurosci, 23(33):10645-10649, Nov 2003.

[28] M. Ju and D. Wray. Molecular identification and characterisation of the human eag2 potassium channel. FEBS Lett, 524(1-3):204-210, Jul 2002.

[29] L. A. Jurado, P. S. Chockalingam, and H. W. Jarrett. Apocalmodulin. Physiol Rev, 79(3):661-682, Jul 1999.

[30] S. Kaech, H. Parmar, M. Roelandse, C. Bornmann, and A. Matus. Cytoskeletal microdifferentiation: a mechanism for organizing morphological plasticity in dendrites. Proc Natl Acad Sci U S A, 98(13):7086-7092, Jun 2001.

[31] W. D. Kaplan and W. E. Trout. The behavior of four neurological mutants of Drosophila. Genetics, 61(2):399-409, Feb 1969.

[32] S. Kuegler, L. Meyn, H. Holzmueller, E. Gerhardt, S. Isenmann, J. B. Schulz, and M. Baehr. Neuron-specific expression of therapeutic proteins: evaluation of different cellular promoters in recombinant adenoviral vectors. Mol Cell Neurosci, 17(1):78-96, Jan 2001.

[33] S. Kupershmidt, T. Yang, S. Chanthaphaychith, Z. Wang, J. A. Towbin, and D. M. Roden. Defective human Ether--go-go-related gene trafficking linked to an endoplasmic reticulum retention signal in the $\mathrm{C}$ terminus. $J$ Biol Chem, 277(30):27442-27448, Jul 2002.

[34] J. Kyte and R. F. Doolittle. A simple method for displaying the hydropathic character of a protein. J Mol Biol, 157(1):105-132, May 1982.

[35] J. R. Lakowicz. Principles of Fluorescence Spectroscopy. Springer, 1999.

[36] K. Luby-Phelps and D. L. Taylor. Subcellular compartmentalization by local differentiation of cytoplasmic structure. Cell Motil Cytoskeleton, 10(1-2):2837, 1988.

[37] J. Ludwig, D. Owen, and O. Pongs. Carboxy-terminal domain mediates assembly of the voltage-gated rat ether--go-go potassium channel. EMBO J, 16(21):6337-6345, Nov 1997.

[38] J. Ludwig, H. Terlau, F. Wunder, A. Brueggemann, L. A. Pardo, A. Marquardt, W. Stuehmer, and O. Pongs. Functional expression of a rat homologue of the voltage gated either go-go potassium channel reveals differences in selectivity and activation kinetics between the Drosophila channel and its mammalian counterpart. EMBO J, 13(19):4451-4458, Oct 1994.

[39] J. Ludwig, R. Weseloh, C. Karschin, Q. Liu, R. Netzer, B. Engeland, C. Stansfeld, and O. Pongs. Cloning and functional expression of rat eag2, a new 
member of the ether--go-go family of potassium channels and comparison of its distribution with that of eag1. Mol Cell Neurosci, 16(1):59-70, Jul 2000.

[40] M. Margeta-Mitrovic, Y. N. Jan, and L. Y. Jan. A trafficking checkpoint controls GABA(B) receptor heterodimerization. Neuron, 27(1):97-106, Jul 2000 .

[41] A. Matus and B. Riederer. Microtubule-associated proteins in the developing brain. Ann N Y Acad Sci, 466:167-179, 1986.

[42] T. B. McAnaney, W. Zeng, C. F. E. Doe, N. Bhanji, S. Wakelin, D. S. Pearson, P. Abbyad, X. Shi, S. G. Boxer, and C. R. Bagshaw. Protonation, photobleaching, and photoactivation of yellow fluorescent protein (YFP 10C): a unifying mechanism. Biochemistry, 44(14):5510-5524, Apr 2005.

[43] W. E. Meador, A. R. Means, and F. A. Quiocho. Target enzyme recognition by calmodulin: 2.4 A structure of a calmodulin-peptide complex. Science, 257(5074):1251-1255, Aug 1992.

[44] P. G. Mermelstein, K. Deisseroth, N. Dasgupta, A. L. Isaksen, and R. W. Tsien. Calmodulin priming: nuclear translocation of a calmodulin complex and the memory of prior neuronal activity. Proc Natl Acad Sci U SA, 98(26):15342-15347, Dec 2001.

[45] R. D. Mitra, C. M. Silva, and D. C. Youvan. Fluorescence resonance energy transfer between blue-emitting and red-shifted excitation derivatives of the green fluorescent protein. Gene, 173(1 Spec No):13-17, 1996.

[46] A. Miyawaki, J. Llopis, R. Heim, J. M. McCaffery, J. A. Adams, M. Ikura, and R. Y. Tsien. Fluorescent indicators for $\mathrm{Ca} 2+$ based on green fluorescent proteins and calmodulin. Nature, 388(6645):882-887, Aug 1997.

[47] W. E. Moerner, E. J. Peterman, S. Brasselet, S. Kummer, and R. M. Dickson. Optical methods for exploring dynamics of single copies of green fluorescent protein. Cytometry, 36(3):232-238, Jul 1999.

[48] J. R. Monck, I. M. Robinson, A. L. Escobar, J. L. Vergara, and J. M. Fernandez. Pulsed laser imaging of rapid $\mathrm{Ca} 2+$ gradients in excitable cells. Biophys J, 67(2):505-514, Aug 1994.

[49] V. Moreau, F. Frischknecht, I. Reckmann, R. Vincentelli, G. Rabut, D. Stewart, and M. Way. A complex of N-WASP and WIP integrates signalling cascades that lead to actin polymerization. Nat Cell Biol, 2(7):441-448, Jul 2000.

[50] M. X. Mori, M. G. Erickson, and D. T. Yue. Functional stoichiometry and local enrichment of calmodulin interacting with $\mathrm{Ca} 2+$ channels. Science, 304(5669):432-435, Apr 2004.

[51] T. Nagai, K. Ibata, E. S. Park, M. Kubota, K. Mikoshiba, and A. Miyawaki. A variant of yellow fluorescent protein with fast and efficient maturation for cell-biological applications. Nat Biotechnol, 20(1):87-90, Jan 2002. 
[52] J. Napp, F. Monje, W. Stuehmer, and L. A. Pardo. Glycosylation of Eag1 (Kv10.1) potassium channels: intracellular trafficking and functional consequences. J Biol Chem, 280(33):29506-29512, Aug 2005.

[53] R. Neher and E. Neher. Optimizing imaging parameters for the separation of multiple labels in a fluorescence image. J Microsc, 213(Pt 1):46-62, Jan 2004.

[54] R. A. Neher and E. Neher. Applying spectral fingerprinting to the analysis of FRET images. Microsc Res Tech, 64(2):185-195, Jun 2004.

[55] T. Occhiodoro, L. Bernheim, J. H. Liu, P. Bijlenga, M. Sinnreich, C. R. Bader, and J. Fischer-Lougheed. Cloning of a human ether-a-go-go potassium channel expressed in myoblasts at the onset of fusion. FEBS Lett, 434(1-2):177-182, Aug 1998.

[56] T. G. Oertner and A. Matus. Calcium regulation of actin dynamics in dendritic spines. Cell Calcium, 37(5):477-482, May 2005.

[57] H. Okamoto and K. Ichikawa. A model for molecular mechanisms of synaptic competition for a finite resource. Biosystems, 55(1-3):65-71, Feb 2000.

[58] A. Palfi, S. Vizi, and K. Gulya. Differential distribution and intracellular targeting of mRNAs corresponding to the three calmodulin genes in rat brain. A quantitative in situ hybridization study. J Histochem Cytochem, 47(5):583600, May 1999.

[59] L. A. Pardo, D. del Camino, A. Snchez, F. Alves, A. Brueggemann, S. Beckh, and W. Stuehmer. Oncogenic potential of EAG $\mathrm{K}(+)$ channels. EMBO J, 18(20):5540-5547, Oct 1999.

[60] A. R. Rhoads and F. Friedberg. Sequence motifs for calmodulin recognition. FASEB J, 11(5):331-340, Apr 1997.

[61] M. A. Rizzo, G. H. Springer, B. Granada, and D. W. Piston. An improved cyan fluorescent protein variant useful for FRET. Nat Biotechnol, 22(4):445-449, Apr 2004.

[62] G. A. Robertson, J. M. Warmke, and B. Ganetzky. Potassium currents expressed from Drosophila and mouse eag cDNAs in Xenopus oocytes. Neuropharmacology, 35(7):841-850, 1996.

[63] V. A. Romoser, P. M. Hinkle, and A. Persechini. Detection in living cells of Ca2+-dependent changes in the fluorescence emission of an indicator composed of two green fluorescent protein variants linked by a calmodulin-binding sequence. A new class of fluorescent indicators. J Biol Chem, 272(20):1327013274, May 1997.

[64] B. L. Sabatini, M. Maravall, and K. Svoboda. Ca(2+) signaling in dendritic spines. Curr Opin Neurobiol, 11(3):349-356, Jun 2001.

[65] B. L. Sabatini, T. G. Oertner, and K. Svoboda. The life cycle of $\mathrm{Ca}(2+)$ ions in dendritic spines. Neuron, 33(3):439-452, Jan 2002. 
[66] M. J. Saganich, E. Machado, and B. Rudy. Differential expression of genes encoding subthreshold-operating voltage-gated $\mathrm{K}+$ channels in brain. J Neurosci, 21(13):4609-4624, Jul 2001.

[67] Y. Saimi and C. Kung. Calmodulin as an ion channel subunit. Annu Rev Physiol, 64:289-311, 2002.

[68] T. Schikorski and C. F. Stevens. Quantitative ultrastructural analysis of hippocampal excitatory synapses. J Neurosci, 17(15):5858-5867, Aug 1997.

[69] R. Schoenherr, G. Gessner, K. Loeber, and S. H. Heinemann. Functional distinction of human EAG1 and EAG2 potassium channels. FEBS Lett, 514(23):204-208, Mar 2002.

[70] R. Schoenherr, K. Loeber, and S. H. Heinemann. Inhibition of human ether go-go potassium channels by $\mathrm{Ca}(2+)$ /calmodulin. EMBO J, 19(13):3263-3271, Jul 2000.

[71] N. C. Shaner, P. A. Steinbach, and R. Y. Tsien. A guide to choosing fluorescent proteins. Nat Methods, 2(12):905-909, Dec 2005.

[72] K. Shen and T. Meyer. Dynamic control of CaMKII translocation and localization in hippocampal neurons by NMDA receptor stimulation. Science, 284(5411):162-166, Apr 1999.

[73] C. Stansfeld, J. Ludwig, J. Roeper, R. Weseloh, D. Brown, and O. Pongs. A physiological role for ether--go-go K+ channels? Trends Neurosci, 20(1):1314, Jan 1997.

[74] C. E. Stansfeld, J. Roeper, J. Ludwig, R. M. Weseloh, S. J. Marsh, D. A. Brown, and O. Pongs. Elevation of intracellular calcium by muscarinic receptor activation induces a block of voltage-activated rat ether--go-go channels in a stably transfected cell line. Proc Natl Acad Sci U S A, 93(18):9910-9914, Sep 1996.

[75] M. Stocker. Ca(2+)-activated K+ channels: molecular determinants and function of the SK family. Nat Rev Neurosci, 5(10):758-770, Oct 2004.

[76] K. F. Sullivan. Structure and utilization of tubulin isotypes. Annu Rev Cell Biol, 4:687-716, 1988.

[77] H. Terlau, S. H. Heinemann, W. Stuehmer, O. Pongs, and J. Ludwig. Amino terminal-dependent gating of the potassium channel rat eag is compensated by a mutation in the S4 segment. J Physiol, 502 ( Pt 3):537-543, Aug 1997.

[78] H. Terlau, J. Ludwig, R. Steffan, O. Pongs, W. Stuehmer, and S. H. Heinemann. Extracellular Mg2+ regulates activation of rat eag potassium channel. Pflugers Arch, 432(2):301-312, Jun 1996.

[79] R. Thorogate and K. Toeroek. Ca2+-dependent and -independent mechanisms of calmodulin nuclear translocation. J Cell Sci, 117(Pt 24):5923-5936, Nov 2004. 
[80] S. L. Toutenhoofd and E. E. Strehler. The calmodulin multigene family as a unique case of genetic redundancy: multiple levels of regulation to provide spatial and temporal control of calmodulin pools? Cell Calcium, 28(2):83-96, Aug 2000.

[81] M. C. Trudeau and W. N. Zagotta. Mechanism of calcium/calmodulin inhibition of rod cyclic nucleotide-gated channels. Proc Natl Acad Sci USA, 99(12):8424-8429, Jun 2002.

[82] R. Y. Tsien. The green fluorescent protein. Annu Rev Biochem, 67:509-544, 1998.

[83] H. Tsutsui, S. Karasawa, H. Shimizu, N. Nukina, and A. Miyawaki. Semirational engineering of a coral fluorescent protein into an efficient highlighter. EMBO Rep, 6(3):233-238, Mar 2005.

[84] J. L. Tubbs, J. A. Tainer, and E. D. Getzoff. Crystallographic structures of Discosoma red fluorescent protein with immature and mature chromophores: linking peptide bond trans-cis isomerization and acylimine formation in chromophore maturation. Biochemistry, 44(29):9833-9840, Jul 2005.

[85] G. Valentin, C. Verheggen, T. Piolot, H. Neel, M. Coppey-Moisan, and E. Bertrand. Photoconversion of YFP into a CFP-like species during acceptor photobleaching FRET experiments. Nat Methods, 2(11):801, Nov 2005.

[86] J. Vreede, M. A. van der Horst, K. J. Hellingwerf, W. Crielaard, and D. M. F. van Aalten. PAS domains. Common structure and common flexibility. J Biol Chem, 278(20):18434-18439, May 2003.

[87] Z. Wang, G. F. Wilson, and L. C. Griffith. Calcium/calmodulin-dependent protein kinase II phosphorylates and regulates the Drosophila eag potassium channel. J Biol Chem, 277(27):24022-24029, Jul 2002.

[88] J. Warmke, R. Drysdale, and B. Ganetzky. A distinct potassium channel polypeptide encoded by the Drosophila eag locus. Science, 252(5012):15601562, Jun 1991.

[89] J. W. Warmke and B. Ganetzky. A family of potassium channel genes related to eag in Drosophila and mammals. Proc Natl Acad Sci U S A, 91(8):34383442, Apr 1994.

[90] G. A. Wayman, S. Kaech, W. F. Grant, M. Davare, S. Impey, H. Tokumitsu, N. Nozaki, G. Banker, and T. R. Soderling. Regulation of axonal extension and growth cone motility by calmodulin-dependent protein kinase I. J Neurosci, 24(15):3786-3794, Apr 2004.

[91] J. Weinman, B. D. Gaspera, A. Dautigny, D. P. Dinh, J. Wang, H. Nojima, and S. Weinman. Developmental regulation of calmodulin gene expression in rat brain and skeletal muscle. Cell Regul, 2(10):819-826, Oct 1991. 
[92] D. Weitz, M. Zoche, F. Mueller, M. Beyermann, H. G. Koerschen, U. B. Kaupp, and K. W. Koch. Calmodulin controls the rod photoreceptor CNG channel through an unconventional binding site in the N-terminus of the betasubunit. EMBO J, 17(8):2273-2284, Apr 1998.

[93] H. Wen and I. B. Levitan. Calmodulin is an auxiliary subunit of KCNQ2/3 potassium channels. J Neurosci, 22(18):7991-8001, Sep 2002.

[94] K. L. Yap, J. Kim, K. Truong, M. Sherman, T. Yuan, and M. Ikura. Calmodulin target database. J Struct Funct Genomics, 1(1):8-14, 2000.

[95] E. Yus-Najera, I. Santana-Castro, and A. Villarroel. The identification and characterization of a noncontinuous calmodulin-binding site in noninactivating voltage-dependent KCNQ potassium channels. J Biol Chem, 277(32):2854528553, Aug 2002.

[96] N. Zerangue, B. Schwappach, Y. N. Jan, and L. Y. Jan. A new ER trafficking signal regulates the subunit stoichiometry of plasma membrane K(ATP) channels. Neuron, 22(3):537-548, Mar 1999.

[97] J. Zheng, M. D. Varnum, and W. N. Zagotta. Disruption of an intersubunit interaction underlies Ca2+-calmodulin modulation of cyclic nucleotide-gated channels. J Neurosci, 23(22):8167-8175, Sep 2003.

[98] I. B. Zhulin, B. L. Taylor, and R. Dixon. PAS domain S-boxes in Archaea, Bacteria and sensors for oxygen and redox. Trends Biochem Sci, 22(9):331333, Sep 1997.

[99] U. Ziechner, R. Schoenherr, A.-K. Born, O. Gavrilova-Ruch, R. W. Glaser, M. Malesevic, G. Kuellertz, and S. H. Heinemann. Inhibition of human ether go-go potassium channels by $\mathrm{Ca} /$ calmodulin binding to the cytosolic $\mathrm{N}$ - and C-termini. FEBS J, 273(5):1074-1086, Mar 2006.

[100] T. Zimmermann, J. Rietdorf, A. Girod, V. Georget, and R. Pepperkok. Spectral imaging and linear un-mixing enables improved FRET efficiency with a novel GFP2-YFP FRET pair. FEBS Lett, 531(2):245-249, Nov 2002. 


\section{Acknowledgments}

I would like to express my gratitude to:

Prof. Dr. Walter Stühmer, Prof. Dr. Erwin Neher and Dr. Fred Wouters for accepting to be a part of my thesis advisory committee and for their guidance during my doctoral studies.

Dr. Gertrude Bunt for her committed supervision, perseverance and willingness to teach some biology to a physicist.

The Department of Molecular Biology of Neuronal Signals and, again, its director Walter Stühmer for their continuous support and excellent working environment.

Barbara Scheufler for preparing the primary neuronal cultures with outstanding skill and for her patience and willingness to teach me the procedure.

Anton Karabinos for introducing me to overlay blots and other biochemical techniques.

Ekaterina Papusheva for a variety of reasons, including her critical reading of this thesis.

Milena Ninkovic, Eva Herrero-Herranz, and Jenni Uhlendorf for the use of the fluorescently-labeled constructs, among other things.

The Cell Biophysics Group at the E.N.I.-Göttingen and, again, Fred Wouters for their collaboration in a variety of topics.

Alessandro Esposito for many discussions on image analysis and for the use of ImFluo.

Mišo Mitkovski for help with $\mathrm{AT}_{\mathrm{E}} \mathrm{X}$ templates.

Martin Oheim for all I learned about microscopy during my stay in his lab.

The DFG Graduiertenkolleg 723 for financial support and Prof. Dr. Dr. Detlev Schild for his organizational efforts.

The DFG SFB 406 and its speaker Prof. Dr. Diethelm Richter. 
Dr. Alistair Mathie and Prof. Nick Franks for introducing me to the biophysics of nerve cells.

The Faculty and Coordinators (past and present) of the Göttingen Neurosciences Graduate Program for their effort in organizing a truly remarkable study program.

Anke Hornbach for many reasons.

The two people without whom this thesis would really never exist - my parents.

And everyone who made my time in Göttingen nicer.

José Tiago Gonçalves

Göttingen, 29 May 2006 


\section{Curriculum Vitæ}

\section{Personal Information}

Name:

José Tiago Paiva Figueiredo Gonçalves

Work address:

Max-Planck-Institute for

Experimental Medicine

Molecular Biology of Neuronal Signals

Hermann-Rein-Str. 3

37075 Göttingen

Germany

Telephone:

+495513899654

E-mail:

goncalves@em.mpg.de

Birthplace:

Porto, Portugal

Date of birth:

25 September 1980

Nationality:

Portuguese

\section{Education}

April 2003 - present (PhD Student)

Max-Planck-Institute for Experimental Medicine

Göttingen, Germany

October 2001 - April 2003

University of Göttingen - Neurosciences Graduate

Program MSc Neurosciences

Göttingen, Germany

October 1998 - July 2001

Imperial College, London BSc Physics London, U.K.

July 1998

Escola Secundária António Nobre Certificado de

Estudos Secundários

Porto, Portugal 


\section{Publications}

Trajkovic, K., Dhaunchak A.S., Gonçalves, J.T., Wenzel D., Schneider A., Bunt G. Nave, K.-A., Simons M., Neuron to glia signaling triggers myelin membrane exocytosis from endosomal storage sites. J Cell Biol, 172, 937-948 (2006)

Schapper F., Gonçalves, J.T., Oheim M., Fluorescence imaging with two-photon evanescent wave excitation. Eur Biophys J, 32, 635-643 (2003) 